\title{
LISTA ACTUALIZADA DE LOS MAMÍFEROS DE MÉXICO 2012
}

\author{
Gerardo Ceballos $^{1}$ y Joaquín Arroyo-Cabrales ${ }^{2}$ \\ 1'Instituto de Ecología, UNAM, Ciudad Universitaria, Apartado Postal 70-275, \\ 04510 México, D.F. \\ "Laboratorio de Arqueozoología "M. en C. Ticul Álvarez Solórzano", Subdi- \\ rección de Laboratorios y Apoyo Académico, INAH, Moneda No. 16 Col. \\ Centro, 06006 México, D.F., México. \\ Autor de correspondencia: Gerardo Ceballos, gceballo@ecologia.unam.mx
}

\begin{abstract}
RESUMEN
La diversidad de mamíferos silvestres de México es una de las mayores en el mundo. Estudios sobre taxonomía, sistemática y distribución en los últimos 15 años han modificado sustancialmente el número de especies del país. Aquí se presenta la lista más reciente y actualizada de los mamíferos de México. Este grupo se encuentra representado por 13 órdenes, 46 familias, 202 géneros y 545 especies, de las cuales 169 son endémicas del país. Esta es la primera vez que el número de especies registradas en el país pasa de 540. Se incluyen datos de continentalidad e insularidad, afinidad biogeográfica y estado de conservación de acuerdo tanto al gobierno federal mexicano como con organismos internacionales. En esta actualización se han agregado los mapas de distribución de las especies.
\end{abstract}

PALABRAS CLAVE: México, mamíferos, presencia, distribución, conservación.

\section{ABSTRACT}

The diversity of Mexican mammals is one of the largest in the World. Over the past 15-years studies on taxonomy, systematics, and distribution have modified the former mammal checklist for the country. Here we present an updated checklist that comprise 13 orders, 46 families, 202 genera, and 545 species, of which 169 are endemic to Mexico. In the checlist we include data on the distribution, biogeographic affinities, and conservation status at the National and World level. We also have included distribution maps of all the species.

KEY WORDS: Mexico, mammals, presence, distribution, conservation. 


\section{INTRODUCCIÓN}

La diversidad biológica de México ha sido reconocida ampliamente. Desde la época precolombina, la diversidad de especies y ecosistemas ha sorprendido a los observadores. De hecho, el Barón Alexander Von Humboldt consideró que el rugoso territorio mexicano era un verdadero paraíso biológico. La riqueza de la especies de mamíferos se hizo aparente a fines del siglo XIX cuando las investigaciones de los norteamericanos E. R. Goldman y E. W. Nelson revelaron que el país tenía cientos de especies y que la magnitud de su biodiversidad era mayor que la del resto de Norteamérica combinada (Goldman, 1951). Esta característica única del país no estuvo disponible para los especialistas hasta hace unas pocas décadas, cuando los estudios y la conservación se volvieron de importancia mundial (Wilson y Reeder, 2005).

La información reunida en las décadas recientes acerca de los patrones de distribución de los mamíferos en el mundo ha establecido claramente que México es uno de los países más ricos en especies de mamíferos del mundo (Ceballos y Brown, 1995; Mittermeier et al., 1997). Aunque el territorio mexicano comprende sólo cerca del $1.6 \%$ de la superficie continental del planeta $\left(1,972,547 \mathrm{~km}^{2}\right)$, él mismo sostiene cerca del $11 \%$ de todas las especies de mamíferos. México, Indonesia, Brasil y China son los países que ocupan los primeros lugares en el número de especies, todos ellos con más de 500. Otros países con alta diversidad de mamíferos incluyen Perú, República Democrática del Congo e India. En general, los países con la mayor riqueza específica de mamíferos, son también muy ricos en los otros grupos taxonómicos. En el caso de México, el país ocupa el primer lugar en la riqueza combinada de anfibios y reptiles, el cuarto en plantas vasculares y el onceavo en aves (Groombridge and Jenkins, 2002; Mittermeier and Goettsch, 1992; Mittermeier et al., 1997). Los países que tienen un número de especies excepcionalmente mayor que el esperado son llamados megadiversos y el reconocimiento de su alta diversidad ha proporcionado un fuerte impulso acerca de su importancia mundial (Ceballos and Brown, 1995; Mittermeier et al., 1997).

Además de su riqueza en especies, México tiene un alto porcentaje de especies endémicas, colocándolo en tercer lugar después de Indonesia y Australia. Otros países con un número alto de mamíferos endémicos son Brasil, China, Filipinas, Madagascar y Papua Nueva Guinea (Ceballos y Brown, 1995). El porcentaje de especies endémicas es mayor en los países insulares, como Australia, Filipinas e Indonesia; sin embargo, México ocupa el primer lugar entre los países continentales. Esto es sorprendente porque tiene mucha más especies endémicas que las que se esperarían de su territorio o por su número total de especies (Ceballos y Brown, 1995). Este patrón es relativamente general porque los países con una alta concentración de mamíferos endémicos, tienen también una alta concentración de muchos otros grupos de animales y plantas.

En relación a los mamíferos, diversos autores han compilado listas de los mamíferos de México tanto terrestres como marinos en las últimas dos décadas (Arita and Ceballos, 1997; Aurioles, 1993; Cervantes et al., 1994; Ceballos et al., 2005; Ramírez-Pulido et al., 1983, 1986, 1996, 2005; Salinas and Ladrón de Guevara, 1993; Torres et al., 1995). Sin embargo, desde 2005 cuando se publicó el compendio más actualizado de mamíferos del Mundo (Wilson y Reeder, 2005) han habido muchos cambios, que incluyen la descripción de nuevas especies, elevación de subespecies a nivel de especies y nuevos registros de taxa para el país, lo que ha aumentado de manera significativa la lista de especies de mamíferos de México. Las dinámicas de estos cambios han sido intensas, con cambios en los niveles de familia, género o especie. Por ejemplo, se estableció que los zorrillos pertenecen a una familia separada y que especies de varios géneros como Sorex, Micronycteris, Natalus, Baeodon, Rhogeessa, Molossus, Puma, Spilogale, Tapirus, Pecari, Cratogeomys, Thomomys, Neotoma, Oryzomys y Sylvilagus se describieron, revalidaron o sinonimizaron. 
Cuadro 1. Composición sistemática de los mamíferos de México.

\begin{tabular}{|l|l|l|l|l|}
\hline ORDEN & FAMILIAS & GÉNEROS & ESPECIES & $\begin{array}{c}\text { ESPECIES } \\
\text { ENDÉMICAS }\end{array}$ \\
\hline \multicolumn{5}{|l|}{} \\
\hline DIDELPHIMORPHIA & 1 & 7 & 8 & 1 \\
\hline CINGULATA & 1 & 2 & 2 & 0 \\
\hline PILOSA & 2 & 2 & 2 & 0 \\
\hline SORICOMORPHA & 2 & 6 & 38 & 23 \\
\hline CHIROPTERA & 9 & 68 & 138 & 16 \\
\hline PRIMATES & 1 & 2 & 3 & 0 \\
\hline CARNIVORA & 8 & 27 & 42 & 3 \\
\hline CETACEA & 7 & 25 & 40 & 1 \\
\hline SIRENIA & 1 & 1 & 1 & 0 \\
\hline PERISSODACTYLA & 1 & 1 & 1 & 0 \\
\hline ARCTIODACTYLA & 4 & 8 & 10 & 0 \\
\hline RODENTIA & 8 & 50 & 245 & 118 \\
\hline LAGOMORPHA & 1 & 3 & 15 & 7 \\
\hline & & & & \\
\hline TOTAL & 46 & 202 & 545 & 169 \\
\hline
\end{tabular}

Aquí se presenta una lista actualizada de las especies de mamíferos de México hasta diciembre de 2012. En la lista se incluyen las afinidades biogeográficas, su distribución y el estado de conservación de cada especie.

\section{MÉTODOS}

Esta lista se basa en compilaciones recientes con los cambios que si indican mas abajo (Ceballos et al., 2002b, 2005). Se excluyeron a los roedores múridos exóticos (Mus musculus, Rattus norvergicus and $R$. rattus) y a las especies domésticas con poblaciones silvestres, como los perros, gatos, cabras y burros. La nomenclatura se basó en Wilson y Reeder (2005), con las modificaciones que se señalan más adelante. Los mapas de distribución se basaron Ceballos y Oliva (2005), Ceballos (en prensa) y Medellin et al. (2008), así como información publicada en trabajos específicos que se indican mas adelante. Las especies se clasificaron de acuerdo a su distribución geográfica actual, como: 1) especies mexicanas compartidas con otros países de Norteamérica (NA), 2) especies mexicanas compartidas con otros países de Centro y Sudamérica (SA), 3) espe- cies con grandes áreas de distribución que incluyen tanto Norte, Centro y Sudamérica (AM), 4) especies que son endémicas a Mesoamérica (México y Centroamérica) (MA) y 5) especies mexicanas endémicas (MX). Las especies de las islas fueron compiladas siguiendo las propuestas de Ceballos et al. (2005), con las modificaciones mencionadas abajo. Estas especies fueron descritas como: completamente insulares (I), completamente continentales (C) o insulares-continentales (IC), para las que se distribuyen tanto en islas como el territorio continental. El estado de conservación en México basa en la norma mexicana de especies en peligro (semarnat, 2010), de acuerdo a las siguientes categorías: $E X=$ extinta; $P=E n$ peligro de extinción; $A=$ Amenazada; $P r=$ Protección especial; * = indica que una subespecie está catalogada en alguna categoría de riesgo. El estado de conservación a nivel global se basa en la Union Internacional para la Conservacion de la Naturaleza (IUCN, 2010; http://redlist.org), de acuerdo a las siguientes categorías: $E X=$ Extinta; EW= Extinta en el medio silvestre; $\mathrm{CR}=$ Criticamente amenazada; $\mathrm{EN}=\mathrm{En}$ peligro; $\mathrm{VU}=$ Vulnerable o Amenazada; $L C=$ Bajo riesgo; 
NT = Casi amenazada; $\mathrm{DD}=$ Datos insuficientes . La clasificación de las especies con respecto al trafico se basa en CITES (2010; http://www. cites.org). CITES clasifica a las especies que están sujetas al comercio internacional en tres apéndices: el Apéndice I incluye las especies en peligro de extinción, por lo que se prohíbe el comercio internacional de especímenes, salvo cuando la importación se realiza con fines no comerciales, como para la investigación científica. El Apéndice II incluye las especies que no están necesariamente amenazadas de extinción pero que podrían llegar a estarlo a menos que se controle estrictamente su comercio. Finalmente, el Apéndice III reglamenta el comercio especies que necesitan la cooperación de otros países para evitar la explotación insostenible o ilegal de las mismas.

Las adiciones y modificaciones en la lista de especies mexicanas, tomando como base las listas de Ceballos et al. (2005) y Wilson y Reeder (2005) son las siguientes:

1. El arreglo taxonómico a nivel suprasubfamilar se baso en Wilson y Reeder (2011).

2. Se incluyó a Cryptotis tropicalis separada recientemente de C. parva (Woodman y Croft, 2005).

3. Para la taxonomía y distribución de las especies del género Sorex se siguió a Carraway (2007). Se incluyeron a dos especies nuevas: S. ixtlanensis y S. mediopua y se acepta que S. monticolus y $S$. saussurei monotípicas. Sin embargo, Esteva et al. (2010) señalaron que $S$. saussurei posiblemente representa especies múltiples, lo que se resolverá con estudios futuros.

4. Se siguió la sugerencia de Ludica (2000) de que el nombre correcto de Sturnira ludovici es S. hondurensis.

5. Porter et al. (2007) estudiaron la filogenia molecular del género Micronycteris, concluyendo con la descripción de dos nuevos subgéneros, asignando las especies mexicanas a Micronycteris (Micronycteris) microtis y Micronycteris (Schizonycteris) schmidtorum.

6. Se siguió a Simmon y Voss (1998) quienes revisaron el estado taxonómico de $\mathrm{Mi}$ mon bennettii y M. cozumelae, y propusieron que ambas son válidas y que M. cozumelae se distribuye en México y Centroamérica. Esta propuesta, sin embargo, ha sido cuestionada por Gregorin et al. (2008).

7. Otra controversia que no ha sido resuelta, aún con análisis detallados (Baker et al., 2000; Van Den Bussche et al., 1998; Wetterer et al., 2000), es si Dermanura es un subgénero de o un género hermana de Artibeus. Hallazgos recientes sugieren que hay datos sólidos que apoyan la existencia de dos géneros separados, por lo que aquí se les trató de esa manera (Hoofer et al., 2006; Solari et al., 2009).

8. Se siguieron las propuestas de Koopman (1993, 1994), Simmons (2005) y Hoofer et al. (2008) de considerar a Artibeus intermedius como un sinónimo de $A$. lituratus por la ausencia de caracteres diagnósticos definitivos que separen ambos taxa a nivel específico, contrario a lo que sugieren Davis (1984) y Guerrero et al. (2003).

9. Velazco y Simmons (2011) revisaron la variación del género Vampyrodes con ayuda del DNA mitrocondrial, morfometría y morfología. Con su análisis demuestraron que el género contiene dos especies bien definidas Vampyrodes caraccioli y Vampyrodes mayor, esta última se distribuyen en México pero anteriormente se consideraba como una subespecie de Vampyrodes caraccioli.

10. Se siguió la propuesta de que en México sólo hay una especie del genero Natalus, con base en resultados morfológicos y genéticos de López-Wilchis et al. (2012). Tejedor (2011) reconoció a N. lanatus and N. mexicanus como especies distintas.

11. Durante los pasados 50 años, diversos autores (Hamilton, 1949; Baker y Patton, 1967; Menu, 1984; Horánék y Hanák, 1985/1986; Hoofer y Van Den Bussche, 2003; y Hoofer et al., 2006) han sugerido la falta de un nombre genérico correcto para los taxa del Nuevo Mundo asignados a Pipistrellus. Basados en los análisis que sustentan algunas de las propuestas presentadas, aquí se reconocieron a los géneros Parastrellus y Perimyotis para las especies hesperus y subflavus, respectivamente.

12. Baird et al. (2008) exploraron las rela- 
ciones filogenéticas de los murciélagos del género Rhogeessa por medio de análisis moleculares, demostrando que las especies $R$. alleni y $R$. gracilis son distantes de las otras especies de Rhogeessa. Para explicar ello, propusieron considerar a ambas dentro del género Baeodon, lo que se siguió en este trabajo,

13. Rhoogessa bickhami es una especie críptica recientemente descrita a partir de ejemplares recolectados en la misma localidad tipo que R. genowaysi en Chiapas (Baird et al., 2012).

14. Eger (2007) reconoció que Eumops nanus se distribuye en México en lugar de $E$. bonariensis.

15. Se aceptó la sugerencia de McDonough et al. (2008) acerca de la presencia de Eumops ferox en México en lugar de E. glaucinus.

16. Wozencraft (2005) y Warren et al. (2006) no reconocieron al género Herpailurus y asignaron a la única especie al género Puma, por lo que el nombre actual más aceptado es Puma yagouaroundi.

17. Se siguió la propuesta de Wozencraft (2005) de que los zorrillos listados del sur y sureste de México son Spilogale angustifrons, mientras que la especie $S$. putorius está restringida a los Estados Unidos de América.

18. La foca de Galápagos (Arctocephalus galapagoensis) fue registrada en las costas de Baja California hace poco tiempo (AuriolesGamboa et al., 2004).

19. El león marino de Galápagos (Zalophus wollebaeki) se registró recientemente en Chiapas (Ceballos et al., 2010).

20. Los lobos finos del norte (Callorhinus ursinus) fueron registrados en México hace ya más de una década (Aurioles-Gamboa et al. 1993).

21. El león marino norteño o de Steller (Eumetopias jubatus) se registró recientemente en la costa de Colima (Ceballos et al., 2010).

22. Se aceptó la propuesta de mantener al coatí de Cozumel (Nasua nelsoni) como una especie válida, críticamente en peligro (Cuaron et al., 2004).

23. Se siguió la conclusión de que el ma- pache de las Islas Tres Marías (Procyon lotor insularis) es una subespecie del mapache continental (Helgen y Wilson, 2005).

24. Groves y Grubb (2011) revisaron de manera detallada las relaciones taxonómicas de los artiodáctilos y los perisodáctilos, basados en los datos morfológicos, citogenéticos y moleculares recientes, así como en las observaciones personales de los autores, todo lo cual apoya sus nuevas propuestas. Especialmente, para México algunos de los cambios incluyen el uso del género Tapirella para el tapir centroamericano, el reconocimiento de dos especies de jabalí de collar, Pecari angulatus y P. crassusm y, el uso del nombre genérico Bos para el bisonte americano. Sin embargo, tales propuesta no han sido aceptadas ampliamente, por lo que no se han usado en este trabajo.

25. Se aceptaron las sugerencias de Geist (1998) y Randi et al. (2001) de reconocer como especie distinta a Cervus elaphus de Europa y Cervus canadensis de Norteamérica.

26. Helgen et al. (2009) revisaron en detalle los ejemplares norteamericanos asignados previamente al género Spermophilus. Basados en varios conjuntos de datos (morfológicos, citogenéticos, ecológicos y conductuales), dichos autores reconocen para México a los siguientes géneros y especies: Notocitellus annulatus, $N$. adocetus, Otospermophilus variegatus, Callospermophilus madrensis, Ictidomys mexicanus e I. parvidens, Xerospermophilus perotensis, $X$. spilosoma, $X$. tereticaudus. Spermophilus sensu stricto estaría restringida al norte de la Región Paleárctica.

27. La revisión sistemática de Cratogeomys merriami por Hafner et al. (2005) permitió reconocer que $C$. merriami es un complejo formado por tres especies, C. merriami, C. fulvescens y $C$. perotensis.

28. Hafner et al. (2008) hicieron la descripción formal de Cratogeomys goldmani.

29. Álvarez-Castañeda (2010) analizó a través de métodos filogeográficos el complejo Thomomys bottae-umbrinus de Estados Unidos de América y México. Basado en los datos genéticos y moleculares, el autor concluye que en el complejo hay ocho especies, seis de 
las cuales se distribuyen en México: T. anitae, T. atrovarius, T. bottae, T. chihuahuae, T. fulvus y $T$. umbrinus. La última especie se halla en la mayor parte del país, mientras que las otras cinco proceden del norte de México En este trabajo, se acepta la recomendación de Hafner et al. (2011) de que la propuesta de Alvarez-Castañeda (2010) requiere el apoyo de otros conjuntos de datos.

30. Hafner et al. (2011) recientemente revisaron las tuzas del noroeste de México, que habitan el matorral xerófilo a lo largo de la vertiente Pacífica de la Sierra Madre Occidental, desde el norte de Sinaloa al oeste de Durango, noroeste de Jalisco y occidente de Nayarit, otorgándoles una nueva asignación taxonómica, Thomomys atrovarius. Aquí se acepto dicha propuesta.

31. Mathis et al., a (en prensa) volvieron a asignarle estatus especifico a Thomomys sheldoni.

32. Mark Haffner (com. pers.) esta describiendo una nueva especie de Thomomys del noreste de Nayarit, que aquí incluimos como Thomomys nayarensis (Mathis et al., b en prensa).

33. Basados en datos moleculares Hafner et al. (2007) concluyeron que el género Liomys es parafilético con Heteromys, lo que situaría a Liomys como su sinónimo. No se siguió aquí tal recomendación hasta que sea ampliamente aceptada.

34. Weskler (2006), basado en una extensa revisión de datos morfológicos y moleculares, propuso una nueva hipótesis filogenética para las especies dentro de la Tribu Oryzomini, proponiendo que dentro del género recientemente descrito Handleyomys Voss et al., 2002, varias especies reconocidas previamente como Oryzomys, deberían ser reubicadas, incluyendo O. alfaroi, O. chapmani, O. melanotis, O. rhapdops, O. rostratus y $O$. saturatior. Dicha propuesta también fue apoyada por Weksler et al. (2006), a diferencia de Weksler y Percequillo (2011), quienes consideran que dichas especies pertenecen a dos grupos de especies que no han sido nombrados. Aquí se ha mantenido a esas especies en el genero Oryzomys hasta que haya un consenso sobre su situación.

35. Carleton y Arroyo (2009) y Hanson et al. (2010) propusieron el estado taxonómico actual dentro del complejo Oryzomys palustris, reconociendo seis especies para México: $O$. albiventer, O. couesi, O. peninsulae, O. mexicanus, O. nelsoni y O. texensis. Por otro lado, Musser y Carleton (2005) consideraron que Oryzomys palustris no se halla en México.

36. Ruedas y Bravo-Salazar (2007) basados en estudios de cariotipos, dividieron Sylvilagus brasiliensis gabbi en dos especies: $S$. gabbi que se distribuye de Panamá hacia el norte y, S. brasiliensis que se halla hacia el sur de Panamá. La subespecie para México es S.g. truei.

\section{RESULTADOS Y DISCUSIÓN}

\section{Composición de Especies, Diversi- dad y Distribución}

México tiene 550 especies de mamíferos que incluye a 201 géneros, 46 familias y 13 órdenes (Cuadro 1, Anexo 1). Los roedores y los murciélagos son los órdenes más ricos en especies, que contribuyen con más del $70 \%$ de todas las especies; les siguen carnívoros, cetáceos, insectívoros y lagomorfos (Cuadro 1, Anexo 1). En general, los géneros están representados por dos especies; sin embargo, los géneros Chaetodipus, Cryptotis, Myotis, Neotoma, Peromyscus, Reithrodontomys y Sorex, son especialemete ricos en especies. Aunque el conocimiento de los mamíferos en México tiene una tradición larga, éste ha aumentado rápidamente en los años recientes y se espera que aumente considerablemente en los siguientes años, al igual que en el resto del planeta, por el advenimiento de técnicas especializadas como la genética molecular (Ceballos y Ehrlich, 2009; Medellín y Soberón, 1999; Patterson, 2001).

Aunque no existe una compilación actualizada sobre el número de mamiferos de todos los paises del mundo, México se encuentra, sin lugar a dudas, entre los cinco paises más diversos, con China, Indonesia, Brasil y Perú (Ceballos y Brown, 1995; Mittermeier et al., 1997; Smith and Xie, 2008). 
El número de especies endémicas es considerable (31\%, 170 spp). Además, aproximadamente el $4 \%$ de los géneros (Tlacuatzin, Megasorex, Musonycteris, Pappogeomys, Zygogeomys, Osgoodomys, Megadontomys, Nelsonia, Neotomodon, Xenomys, Hodomys, Romerolagus) son endémicos al país (también ver Ceballos y Rodríguez, 1993; Ceballos et al., 1998; Escalante et al. 2003; Ramírez-Pulido y Müdespacher, 1987) Las especies endémicas pertenecen a 7 órdenes y 14 familias; la mayoría (116 spp; 68\%) son roedores. Esta alta concentración de especies endémicas coloca a México como uno de los paises continentales con mayor porcentaje de especies endémicas, comparable a paises insulares (Ceballos y Brown, 1997). La fauna restante es una combinación de elementos neotropicales, neárticos o especies compartidas que constituyen dos tercios de las especies mexicanas (ver Alvarez y de La Chica, 1974; Escalante et al., 2003; Ortega y Arita, 1998). Otros grupos de plantas y animales presentan patrones similares (Ramammorthy et al., 1993). Es decir, la fauna mexicana es el resultado de la combinación de elementos neárticos y neotropicales de países megadiversos.

\section{Conservación de especies}

Los problemas ambientales en Mexico son severos (Challender, 1998; Kernan et al., 2012). Se ha reportado que 8 especies se han extinguido en tiempos históricos. Adicionalmente, $238(40 \%)$ están clasificadas que enfrentan problemas de conservación (Ceballos, 1993; Ceballos y Oliva, 2005). Los números y proporciones de taxa extintos y que están en peligro indican que México también está entre los países con mayores problemas en el mundo en estas categorías (Ceballos y Brown, 1995; IUCN, 2012).

Las especies extintas documentadas incluyen cuatro especies insulares de roedores y un pinnípedo. Todos los roedores, incluso Peromyscus pembertoni de la isla de San Pedro Nolasco, Neotoma anthonyi de la isla de Todos Santos, Neotoma bunkeri de las islas de Coronado, y Oryzomys nelsoni y Peromyscus madrensis de las islas de Tres Marías, desaparecieron como consecuencia de la introducción de ratas domésticas (Rattus spp), ratones (Mus musculus) y gatos (Felis catus) (Ceballos y Navarro, 1991; Lawlor, 1983; Mellink, 1992; Smith et al., 1993; Wilson, 1991). Se tienen datos que sugieren que dos especies adicionales pueden estar extintas: Peromyscus guardia de las islas Ángel de la Guarda, Mejía, Granito y Estanque (Mellink et al., 2002) y Dipodomys gravipes del Valle de San Quintin en Baja California (Ceballos y Rodríguez, 1993; E. Mellink, com. pers., 2001). La foca monje del Caribe (Monachus tropicalis) que habitaba en las aguas de Cuba, Jamaica y la Península de Yucatán, se extinguió alrededor de 1952 (Cole et al., 1994; Villa-R. et al., 1986). Adicionalmente, Myotis planiceps y M. milleri eran consideradas extintas por la IUCN (Hilton-Taylor, 2004; IUCN, 2012).

Sin embargo, M. milleri está considerada como una subespecie de $M$. evotis (Manning, 1993) y M. planiceps fue recientemente reencontrada (Arroyo-Cabrales et al., 2005).

Aunque seis especies fueron consideradas como extirpadas de México por Ceballos y Navarro (1991), recientemente se encontró una de ellas, otra fue reintroducida con éxito y una más recolonizó espontáneamente en México. Hasta hace poco, el bisonte (Bison bison) se creía extirpado México (Anderson, 1972; Ceballos y Navarro, 1991; Leopold, 1965); sin embargo, una población salvaje remanente a lo largo de la frontera de Chihuahua con Nuevo México, se descubrió a principios de los 1990 (Pacheco et al., 2002). En cualquier caso, la especie debe ser considerada como críticamente en peligro. El wapití (Cervus elaphus) probablemente se extirpó a principios del último siglo (Leopold, 1959) pero ha sido reintroducido con éxito en Coahuila (Robles Gil et al., 1993). La nutria marina (Enhydra lutris) desapareció a principios de este siglo de las aguas mexicanas (Ceballos y Navarro, 1991); sorprendentemente, algunos individuos se han encontrado cerca de la Isla de Cedros y de la Bahía Magdalena, en la costa de la Península de Baja California (Gallo, 1997; Rodríguez-Jaramillo y Gendron, 1996). En 1950 la nutria de río norte- 
ña (Lontra canadensis) había desaparecido de los ríos Colorado y Bravo (Ceballos y Navarro, 1991); sin embargo, hay una grabación reciente de una nutria de agua dulce en Tamaulipas (G. Ceballos, obs. pers.) que representa a esta especie. El último oso pardo mexicano (Ursus arctos horribilis) se cazó en los 1960s en la Sierra Nido, Chihuahua (Brown, 1985). El lobo mexicano (Canis lupus baileyi) está extinto en libertad, pero unos pocos sobreviven en cautiverio (Ceballos y Navarro, 1991).

Los mamíferos mexicanos están subrepresentados en las listas internacionales de especies con problemas de conservación. Ciento noventa y ocho especies están consideradas por la nueva legislación mexicana como en peligro, amenazadas o bajo protección especial (SEMARNAP, 2002), mientras que sólo 61 mamíferos mexicanos están incluidos en CITES y 131 en las listas de IUCN. Las diferencias más obvias están en los casos que consideran a mamíferos pequeños. Por ejemplo, 36 murciélagos y 14 insectívoros son considerados por SEMARNAT, mientras que ninguno está incluido en CITES y sólo 24 (14 murciélagos y 10 insectívoros) en IUCN. De manera similar, CITES considera tres roedores mexicanos, IUCN incluye $67 \mathrm{y}$ SEMANRNAT enlista 64 especies de preocupación. Recíprocamente, la mayoría de los cetáceos mexicanos está incluido en CITES, mientras que este grupo está sub-representado en la lista de IUCN. El sesgo en los listados de CITES indudablemente se relaciona con el objetivo de esa convención, proteger sólo aquellas especies que se sujetan al comercio internacional, que son principalmente las especies grandes. Las regulaciones internacionales protegen algunas especies de mamíferos mexicanos, pero estas regulaciones son claramente inadecuadas si la protección de la diversidad del país es la meta de la conservación.

\section{CONCLUSIONES}

Las 550 especies de mamíferos terrestres y marinos conocidas actualmente para México lo convierten en un país megadiverso y, a la vez, le implican una gran responsabilidad para la conservación de dicha diversidad. Es necesario que se realicen estudios más detallados de muchos de los taxa para conocer su biología, ecología y estado de conservación.

\section{Agradecimientos}

Queremos hacer patente nuestro más sincero agradecimiento a Jesús Pacheco, Yolanda Domínguez Castellanos y Lourdes Martínez-Estévez por su apoyo en la revisión de la lista de especies, mapas y bibliografía para este artículo. Agradecemos también a nuestras instituciones por su apoyo para desarrollar nuestro trabajo cotidiano.

\section{Literatura Citada}

Álvarez-Castañeda, S.T. 2010. Phylogenetic structure of the Thomomys bottae-umbrinus complex in North America. Molecular Phylogenetics and Evolution, 54: 671-679.

Arita, H.T. y G. Ceballos. 1997. Los mamíferos de México: Distribución y estado de conservación. The mammals of Mexico: Distribution and conservation status. Revista Mexicana de Mastozoología, 2: 33-71.

Aurioles, G.D. 1993. Biodiversidad y estado actual de los mamíferos marinos en México. Revista de la Sociedad Mexicana de Historia Natural, 44:397-412.

Aurioles Gamboa, D., J. Urbán Ramírez y B. Morales Vela. 1993. Programa nacional de investigación sobre mamíferos marinos. Pp. 139-159, en: Biodiversidad marina y costera de México (S.I. Salazar y N.E. González, editores). Comisión Nacional para el Conocimiento y Uso de la Biodiversidad y Centro de Investigaciones de Quintana Roo, México, 865 pp.

Aurioles-Gamboa D., Y. Schramm y S. Mesnick. 2004. Galapagos fur seals, Arctocephalus galapagoensis, in Mexico. Latin American Journal of Aquatic Mammals, 3:77-90.

Baird, A.B., D.M. Hills, J.C. Patton y J.W. Bickham. 2008. Evolutionary history of the genus Rhogeessa (Chiroptera: Vespertilionidae) as revelated by mitochondrial DNA sequences. Journal of Mammalogy, 89:744-754.

Baird, A.B., M.R. Marchán-Rivadeneira, S.G. Pérez y R.J. Baker. 2012. Morphological analysis and description of two new species of Rhogeessa (Chiroptera: Vespertilionidae) from the Neotropics. Occasional Papers, The Museum of Texas Tech University, 307:1-25.

Baker, R.J. y J.L. Patton. 1967. Karyotypes and karyotypic variation of North American vespertilionid bats. Journal of Mammalogy, 48: 270-286.

Baker, R.J., C.A. Porter, J.C. Patton y R.A. Van Den Bussche. 2000. Systematics of bats of the Family Phyllostomidae based on RAG2 DNA sequences. Occasional Papers, The Museum of Texas Tech University, 202: i+1-16. 
Carleton, M.D. y J. Arroyo-Cabrales. 2009 Chapter 3. Review of the Oryzomys couesi complex (Rodentia: Cricetidae: Sigmodontinae) in Western Mexico. Pp. 93127, en: Systematic Mammalogy: contibutions in honor of Guy G. Musser (R.S. Voss y M.D. Carleton, Eds.). Bulletin of the American Museum of Natural History, 331: 1- 450.

Carraway, L.N. 2007. Shrews (Eulypotyphla: Soricidae) of México. Monographs of the Western North American Naturalist, 3: 1-91.

Ceballos, G. En prensa. Mammals of Mexico. Johns Hokins Press, Baltimore.

Ceballos, G. y G. Oliva (Coords.). 2005. Los mamíferos silvestres de México. Comisión Nacional para el Conocimiento y Uso de la Biodiversidad y Fondo de Cultura Económica, México, D.F.

Ceballos, G., J. Arroyo-Cabrales y R. A. Medellín. 2002. The mammals of México: composition, distribution, and conservation. Occasional Papers, The Museum of Texas Tech University, 218: 1-27.

Ceballos, G., J. Arroyo-Cabrales, R.A. Medellín y Y. Domínguez-Castellanos. 2005. Lista actualizada de los mamíferos de México. Revista Mexicana de Mastozoología, 9: 20-70.

Ceballos, G., P. Manzano, F.M. MéndezHarclerode, M.L. Haynie, D.H. Walker y R.D. Bradley. 2010. Geographic distribution, genetic diversity, and conservation status of the southern flying squirrel (Glaucomys volans) in México. Occasional Papers, Museum of Texas Tech University, 299: 1-15.

Cervantes, F.A., A. Castro-Campillo y J. Ramírez-Pulido. 1994. Mamíferos terrestres nativos de México. Anales del Instituto de Biología, Universidad Nacional Autónoma de México, Serie Zoología, 65: 177-190.

Challenger, A. 1998. Utilización y conservación de los ecosistemas terrestres de México. Pasado, presente y futuro. Comisión Nacional para el Conocimiento y uso de la Biodiversidad, México, D.F.

Cuarón, A.D., M.A, Martínez-Morales, K.W. McFadden, D. Valenzuela y M.E. Gompper. 2004. The status of dwarf carnivores on Cozumel Island, México. Biodiversity and Conservation, 13: 317-331.

Davis, W.B. 1984. Review of the large fruit-eating bats of the "Artibeus lituratus" complex (Chiroptera: Phyllostomidae) in Middle America. Occasional Papers, The Museum of Texas Tech University, 93: 1-16.

Eger, J.L. 2007(2008). Family Molossidae P. Gervais, 1856. Pp. 399-440, en: Mammals of South America. Volume 1. Marsupials, xenarthrans, shrews, and bats (A. L. Gardner, Ed.). Chicago: The University Chicago Press, Chicago.
Escalante, T., D. Espinosa y J. Morrone. 2003. Using parsimony analysis of endemicity to analyze the distribution of Mexican mammals. The Southwestern Naturalist, 48:563-578.

Esteva, M., F.A. Cervantes, S.V. Brant y J.A. Cook. 2010. Molecular phylogeny of longtailed shrews (genus Sorex) from México and Guatemala. Zootaxa, 2615: 47-65.

Gregorin, R., G.L. Capusso y V.R. Furtado. 2008. Geographic distribution and morphological variation in Mimon bennettii (Chiroptera, Phyllostomidae). Iheringia, Série Zoologia, Porto Alegre, 98: 404-411.

Groves, C. y P. Grubb. 2011. Ungulate Taxonomy. The Johns Hopkins University Press, Baltimore, $i x+317 p p$.

Guerrero, J.A., E. de Luna y C. SánchezHernández. 2003. Morphometrics in the quantification of character state identity for the assessment of primary homology: an analysis of character variation of the genus Artibeus (Chiroptera: Phyllostomidae). Biological Journal of the Linnaean Society, 80: 45-55.

Hafner, M.S., J.E. Leight, D.J. Hafner, S.V. Brant, T.A. Spradling y J.W. Demastes. 2005. Cryptic species in the Mexican pocket gopher Cratogeomys merriami. Journal of Mammalogy, 86: 1095-1108.

Hafner, J.C., J.E. Leight, D.J. Hafner, M.S. Hafner, E. Reddington, D.S. Rogers y B.R. Riddle. 2007. Basal clades and molecular systematic of heteromyid rodents. Journal of Mammalogy, 88: 1129-1145.

Hafner, D.J., M.S. Hafner, G.L. Hasty, T.A. Spredling y J.W. Demastes. 2008. Evolutionary relationships of pocket gophers (Cratogeomys castanops species group) of the Mexican Altiplano. Journal of Mammalogy, 89: 190-208.

Hafner, M.S., A.R. Gates, V. Mathis, J.W. Demastes y D.J. Hafner. 2011. Redescription of the pocket gopher Thomomys atrovarius from the Pacific coast of mainland Mexico. Journal of Mammalogy, 92: 1367-1382.

Hamilton, W.J. Jr. 1949. The bacula of some North American vespertilionid bats. Journal of Mammalogy, 30: 97-102.

Hanson, J.D., J.L. Indorf, V.J. Swier y R.D. Bradley. 2010. Molecular divergence within the Oryzomys palustris complex: evidence for multiple species. Journal of Mammalogy, 91: 336-347.

Helgen, K.M. y D.E. Wilson. 2005. Cap. 20. A systematic and zoogeographic overview of the raccons of Mexico and Central America. Pp. 221-236, en: Contribuciones mastozoológicas en Homenaje a Bernardo Villa (V. Sánchez-Cordero y R. A. Medellín, Eds.). México: Instituto de Biología, UNAM; Instituto de Ecología, UNAM, CONABIO, Mexico D.F.
Helgen, K.M., F.R. Cole, L.E. Helgen y D.E. Wilson. 2009. Generic revision in the Holartic ground squirrel genus Spermophilus. Journal of Mammalogy, 90: 270-305.

Hoofer, S.R. y R.A. Van Den Bussche. 2003. Molecular phylogenetics of the chiropteran family Vespertilionidae. Acta Chiropterologica, 5 (suplement): 1-63.

Hoofer, S.R., R.A. Van Den Bussche e I. Horàcék. 2006. Generic status of the American pipistrelles (Vespertilionidae) with description of a new genus. Journal of Mammalogy, 87: 981-992.

Hoofer, S.R., S. Solari, P.A. Larsen, R.D. Bradley y R.J. Baker. 2008. Phylogenetics of the fruit-eating bats (Phyllostomidae: Artibeina) Inferred from Mitochondrial DNA Sequences. Occasional Papers, The Museum of Texas Tech University, 277: 1-15.

Horánék, I. y V. Hanák. 1985/1986. Generic status of Pipistrellus savii and comments on classification of the genus Pipistrellus (Chiroptera, Vespertilionidae). Myotis, 23/24: 9-16.

IUCN. 2012. The IUCN list of threatened species. IUCN, Gland, Suiza (http://www.iucnredlist. org/).

Kernan, B., G. Ceballos, S. Solberg, C. Equihua Z., D. Griswold, M. Seager, R.A. Medellín, I. Pisanty Baruch, C. Galicia y E. Ponce Guevara. 2012. Mexico Tropical Forest and Biodiversity Assessment. USAID, Mexico D.F.

Koopman, K.F. 1993. Order Chiroptera. Pp. 137-241, en: Mammal species of the World. A taxonomic and geographic reference (D.E. Wilson and D.A.M. Reeder, eds), Second edition. Smithsonian Institution Press, Washington and London in assoc. American Society of Mammalogists, Lawrence, Kansas.

Koopman, K. F. 1994. Chiroptera: Systematics. en: Handbuch der zoologie: Eine Naturgeschichte der stamme des tierreiches. Band $8=$ Handbook of zoology: A natural history of the Phyla of the Animal Kingdom. Vol. 8. Mammalia (Eds. J. Niethammer, H. Schliemann, and D. Starck.). Walter de Gruyter, Berlin y New York.

Lopez-Wilchis, R.L., M. Guevara-Chumacero, N.A. Perez, J. Juste, C. Ibañez y I.D.L.A. Barriga-Sosa. 2012. Taxonomic status assessment of the Mexican populations of Funnel-eared bats, genus Natalus (Chiroptera: Natalidae). Acta Chiropterologica, 14: 305316.

Mathis, V.L., M.S. Hafner, D.J. Hafner y J.W. Demastes. a. En prensa. Resurrection and redescription of the pocket gopher Thomomys sheldoni from the Sierra Madre Occidental of Mexico. Journal of Mammalogy.

Mathis, V.L., M.S. Hafner, D.J. Hafner y J W. Demastes. b. En prensa. Thomomys 
nayarensis, a new species of pocket gopher from the Sierra del Nayar, Nayarit, Mexico. Journal of Mammalogy.

McDonough, M.M., L.K. Merman, R.M. Timm, H.H. Genoways, P.A. Larsen y R.J. Baker. 2008. Speciation within bonneted bats (genus Eumops): the complexity of the morphological, mitochondrial, and nuclear data sets in systematics. Journal of Mammalogy, 89: 1306-1315.

Medellín, R.A., H.T. Arita y O. Sánchez H. 2008. Identificación de los murciélagos de México. Claves de campo. Revista Mexicana de Mastozoología, 2: 1-83.

Menu, H. 1984. Révision du statut de Pipistrellus subflavus (F. Cuvier, 1832). Proposition d'un taxon generique noveau: Perimyotis nov. gen. Mammalia, 48: 409-416.

Musser, G.G. y M.D. Carleton. 2005. Superfamily Muroidea. Pp. 894-1534, en: Mammal species of the World. A taxonomic and geographic reference (D.E. Wilson, and D.A.M. Reeder, Eds.). The Johns Hopkins University Press, Baltimore.

Ortega, J. y H.T. Arita. 1998. Neotropical-Nearctic limits in Middle America as determined by distributions of bats. Journal of Mammalogy, 79:772-783.

Porter, C.A., S.R. Hoofer, C.A. Cline, F.G. Hoffmann y R.J. Baker. 2007. Molecular phylogenetics of the phyllostomid bat genus Micronycteris with descriptions of two new subgenera. Journal of Mammalogy, 88: 1205-1215.

Ramírez-Pulido, J., R. López Wilchis, C. Müdespacher e I. Lira. 1983. Lista y bibliografía reciente de los mamíferos de México. Universidad Autónoma Metropolitana-Iztapalapa, Mexico D.F.

Ramírez-Pulido, J., A. Castro-Campillo, J. Arroyo-Cabrales y F.A. Cervantes. 1996. Lista taxonómica de los mamíferos terrestres de México: A taxonomic list of the terrestrial mammals of Mexico. Occasional Papers, The Museum of Texas Tech University, 158: 1-62.

Ramírez-Pulido, J., J. Arroyo-Cabrales y A. Castro-Campillo. 2005. Estado actual y relación nomenclatural de los mamíferos terrestres de México. Acta Zoológica Mexicana (nueva serie), 21: 21-82.

Ruedas, L.A. y J. Salazar-Bravo. 2007. Morphological and chromosomal taxonomic assessment of Sylvilagus brasiliensis gabbi (Leporidae). Mammalia, 71: 63-69.

Salinas, M. y P. Ladrón de Guevara. 1993. Riqueza y diversidad de los mamíferos marinos. Ciencia, número especial, 7:85-93.

Simmons, N.B. 2005. Order Chiroptera. Pp. 312-529, en: Mammal species of the World. A taxonomic and geographic reference (D. E. Wilson y D. A. M. Reeder, Editores), Third edition. The Johns Hopkins University Press, Baltimore.

Simmons, N.B. y R.S. Voss. 1998. The mammals of Paracou, French Guiana: a neotropical lowland rainforest fauna part I. Bats. Bulletin of the American Museum of Natural History, 237: 1-219.

Smith, A.T. y Y. Xie. 2008. A Guide to the Mammals of China. Princeton University Press, Princeton, N.J.

Solari, S.S., R. Hoofer, P.A. Larsen, A.D. Brown, R.J. Bull, J.A. Guerrero, J. Ortega, J.P. Carrera, R.D. Bradley y R.J. Baker. 2009. Operational criteria for genetically defined species: analysis of the diversification of the small fruit-eating bats, Dermanura (Phyllostomidae: Stenodermatinae). Acta Chiropterologica, 11: 279-288.

Tejedor, A. 2011. Systematics of funnel-eared bats (Chiroptera: Natalidae). Bulletin of the American Museum of Natural History, 353: $1-140$.

Torres, G.A., M.C. Esquivel y G. Ceballos. 1995. Diversidad y conservación de los mamíferos marinos de México. Revista Mexicana de Mastozoología, 1:22-43.

Van Den Bussche, R.A., J.L. Hudgeons y R.J. Baker. 1998. Phylogenetic accuracy, stability, and congruence. Relationships within and among the New World bat genera Artibeus, Dermanura, and Koopmania. Pp. 59-71, en: Bat biology and conservation ( $\mathrm{K}$. Thomas $\mathrm{H}$. and P.A. Racey, Eds.). Smithsonian Institution
Press, Washington.

Velazco y S. Simmons. 2011. Systematics and taxonomy of great striped-faced bats of the genus Vampyrodes Thomas, 1900 (Chiroptera: Phyllostomidae). American Museum Novitates, 3710:1-35.

Weskler, M. 2006. Phylogenetic relationships of Oryzomine rodents (Muroidea: Sigmodontinae): separate and combined analyses of morphological and moleculardata. Bulletin of the American Museum of Natural History, 296:1-149.

Weskler, M. y A.R. Percequillo. 2011. Key to the genera of the tribe Oryzomyini (Rodentia: Cricetidae: Sigmodontinae). Mastozoología Neotropical, 18:281-292.

Weskler, M., A.R. Percequillo y R.S. Voss. 2006. Ten new genera of Oryzomyine rodents (Cricetidae: Sigmodontinae). American Museum Novitates, 3537:1-29.

Wetterer, A.L., M.V. Rockman y N.B. Simmons. 2000. Phylogeny of phyllostomids bats (Mammalia: Chiroptera): Data from diverse morphological systems, sex chromosomes, and restriction sites. Bulletin of the American Museum of Natural History, 248: 1-200.

Wilson, D.E. y D.A.M. Reeder. 2005. Mammal species of the World. A taxonomic and geographic reference, Third edition. The Johns Hopkins University Press, Baltimore.

Wilson, D.E. y D.A.M. Reeder. 2011. Class Mammalia Linnaeus, 1758. Pp. 56-60, in: Animal biodiversity: An outline of higherlevel classification and survey of taxonomic richness. (Zhang, Z.-Q. Ed.) Zootaxa, 3148:1237.

Wozencraft, W.C. 2005. Order Carnivora. Pp. 512-628, en: Mammal species of the World. A taxonomic and geographic reference (D.E. Wilson, and D.A.M. Reeder, Eds.), Third edition. The Johns Hopkins University Press, Baltimore. 
Apéndice I. Lista de especies de mamíferos de México.

\begin{tabular}{|c|c|c|c|c|c|}
\hline & \multicolumn{2}{|c|}{ Distribución } & \multicolumn{3}{|c|}{ Estado de Conservación } \\
\hline & Ins/Cont & Continente & México & CITES & $\mathrm{UICN}$ \\
\hline \multicolumn{6}{|l|}{ ORDEN DIDELPHIMORPHIA } \\
\hline \multicolumn{6}{|l|}{ FAMILIA DIDELPHIDAE } \\
\hline \multicolumn{6}{|l|}{ SUBFAMILIA DIDELPHINAE } \\
\hline Chironectes minimus (Zimmermann, 1780) & $\mathrm{C}$ & SA & $\mathrm{P}$ & & LC \\
\hline Didelphis marsupialis Linnaeus, 1758 & C & SA & & & LC \\
\hline Didelphis virginiana Kerr, 1792 & $\mathrm{C}$ & NA & & & LC \\
\hline Marmosa mexicana Merriam, 1897 & $\mathrm{C}$ & MA & & & LC \\
\hline Metachirus nudicaudatus (È. Geoffroy St.-Hilaire, 1803) & C & SA & A & & LC \\
\hline Philander opossum (Linnaeus, 1758) & $\mathrm{C}$ & SA & & & LC \\
\hline Tlacuatzin canescens (J. A. Allen, 1893) & IC & MX & & & LC \\
\hline \multicolumn{6}{|l|}{ SUBFAMILIA CALUROMYINAE } \\
\hline Caluromys derbianus (Waterhouse, 1841) & $\mathrm{C}$ & SA & A & & LC \\
\hline \multicolumn{6}{|l|}{ ORDEN SIRENIA } \\
\hline \multicolumn{6}{|l|}{ FAMILIA TRICHECHIDAE } \\
\hline Trichechus manatus Linnaeus, 1758 & M & AM & $\mathrm{P}$ & 1 & VU \\
\hline \multicolumn{6}{|l|}{ ORDEN CINGULATA } \\
\hline \multicolumn{6}{|l|}{ FAMILIA DASYPODIDAE } \\
\hline \multicolumn{6}{|l|}{ SUBFAMILIA DASYPODINAE } \\
\hline Dasypus novemcinctus Linnaeus, 1758 & IC & AM & & & LC \\
\hline \multicolumn{6}{|l|}{ SUBFAMILIA TOLYPEUTINAE } \\
\hline Cabassous centralis (Miller, 1899) & C & SA & $\mathrm{P}$ & III & DD \\
\hline \multicolumn{6}{|l|}{ ORDEN PILOSA } \\
\hline \multicolumn{6}{|l|}{ FAMILIA CYCLOPEDIDAE } \\
\hline Cyclopes didactylus (Linnaeus, 1758) & $\mathrm{C}$ & SA & $\mathrm{P}$ & & LC \\
\hline \multicolumn{6}{|l|}{ FAMILIA MYRMECOPHAGIDAE } \\
\hline Tamandua mexicana (Saussure, 1860) & $\mathrm{C}$ & SA & $\mathrm{P}$ & III & LC \\
\hline \multicolumn{6}{|l|}{ ORDEN PRIMATES } \\
\hline \multicolumn{6}{|l|}{ FAMILIA ATELIDAE } \\
\hline \multicolumn{6}{|l|}{ SUBFAMILIA MYCETINAE } \\
\hline Alouatta palliata (Gray, 1849) & $\mathrm{C}$ & SA & $\mathrm{P}$ & 1 & LC \\
\hline Alouatta pigra Lawrence, 1933 & $\mathrm{C}$ & MA & $\mathrm{P}$ & 1 & EN \\
\hline \multicolumn{6}{|l|}{ SUBFAMILIA ATELINAE } \\
\hline Ateles geoffroyi Kuhl, 1820 & $\mathrm{C}$ & SA & $\mathrm{P}$ & II & EN \\
\hline \multicolumn{6}{|l|}{ ORDEN LAGOMORPHA } \\
\hline \multicolumn{6}{|l|}{ FAMILIA LEPORIDAE } \\
\hline \multicolumn{6}{|l|}{ SUBFAMILIA LEPORINAE } \\
\hline Lepus alleni Mearns, 1890 & IC & NA & * & & LC \\
\hline
\end{tabular}




\begin{tabular}{|c|c|c|c|c|c|}
\hline \multicolumn{6}{|l|}{ Apéndice I. Continuación... } \\
\hline & \multicolumn{2}{|c|}{ Distribución } & \multicolumn{3}{|c|}{ Estado de Conservación } \\
\hline & Ins/Cont & Continente & México & CITES & UICN \\
\hline Lepus californicus Gray, 1837 & IC & NA & * & & LC \\
\hline Lepus callotis Wagler, 1830 & $\mathrm{C}$ & NA & & & NT \\
\hline Lepus flavigularis Wagner, 1844 & $\mathrm{C}$ & $M X$ & $\mathrm{P}$ & & EN \\
\hline Lepus insularis W. Bryant, 1891 & 1 & $M X$ & $\operatorname{Pr}$ & & NT \\
\hline Romerolagus diazi (Ferrari- Pérez, 1893) & C & $M X$ & $\mathrm{P}$ & 1 & EN \\
\hline Sylvilagus audubonii (Baird, 1858) & $\mathrm{C}$ & NA & & & LC \\
\hline Sylvilagus bachmani (Waterhouse, 1839) & IC & NA & * & & LC \\
\hline Sylvilagus cunicularius (Waterhouse, 1848) & $\mathrm{C}$ & $M X$ & & & LC \\
\hline Sylvilagus floridanus (J. A. Allen, 1890) & $\mathrm{C}$ & NA & & & LC \\
\hline Sylvilagus gabii (J. A. Allen, 1877) & C & SA & & & \\
\hline Sylvilagus graysoni (J. A. Allen, 1877) & I & $M X$ & $\mathrm{P}$ & & EN \\
\hline Sylvilagus insonus (Nelson, 1904) & $\mathrm{C}$ & $\mathrm{MX}$ & $\mathrm{P}$ & & EN \\
\hline Sylvilagus mansuetus Nelson, 1907 & 1 & $M X$ & $\mathrm{P}$ & & $\mathrm{CR}$ \\
\hline Sylvilagus robustus (V. Bailey, 1905) & $\mathrm{C}$ & NA & & & EN \\
\hline \multicolumn{6}{|l|}{ ORDEN RODENTIA } \\
\hline \multicolumn{6}{|l|}{ FAMILIA SCIURIDAE } \\
\hline \multicolumn{6}{|l|}{ SUBFAMILIA SCIURINAE } \\
\hline Ammospermophilus harrisii (Audubon \& Bachman, 1854) & C & NA & & & LC \\
\hline Ammospermophilus insularis Nelson \& Goldman, 1909 & I & MX & A & & \\
\hline Ammospermophilus interpres (Merriam, 1890) & $\mathrm{C}$ & NA & & & LC \\
\hline Ammospermophilus leucurus (Merriam, 1889) & $\mathrm{C}$ & NA & & & LC \\
\hline Callospermophilus madrensis Merriam, 1901 & $\mathrm{C}$ & MX & $\operatorname{Pr}$ & & NT \\
\hline Cynomys ludovicianus (Ord, 1815) & $\mathrm{C}$ & NA & A & & LC \\
\hline Cynomys mexicanus Merriam, 1892 & $\mathrm{C}$ & $M X$ & $\mathrm{P}$ & $\mathrm{I}$ & EN \\
\hline Glaucomys volans (Linnaeus, 1758) & $\mathrm{C}$ & NA & A & & LC \\
\hline Ictidomys mexicanus (Erxleben, 1777) & $\mathrm{C}$ & NA & & & LC \\
\hline Ictidomys parvidens (Mearns, 1896) & $\mathrm{C}$ & NA & & & \\
\hline Neotamias bulleri (J. A. Allen, 1889) & $\mathrm{C}$ & $M X$ & & & $\mathrm{VU}$ \\
\hline Neotamias dorsalis (Baird, 1855) & $\mathrm{C}$ & NA & & & LC \\
\hline Neotamias durangae (J. A. Allen, 1903) & $\mathrm{C}$ & $M X$ & & & LC \\
\hline Neotamias merriami (J. A. Allen, 1889) & $\mathrm{C}$ & NA & $\operatorname{Pr}$ & & LC \\
\hline Neotamias obscurus (J. A. Allen, 1890) & $\mathrm{C}$ & NA & & & LC \\
\hline Notocitellus adocetus (Merriam, 1903) & $\mathrm{C}$ & $M X$ & & & LC \\
\hline Notocitellus annulatus (Audubon \& Bachman, 1842) & $\mathrm{C}$ & $M X$ & & & LC \\
\hline Otospermophilus atricapillus (W. E. Bryant, 1889) & $\mathrm{C}$ & $M X$ & & & EN \\
\hline Otospermophilus beecheyi (Richardson, 1829) & $\mathrm{C}$ & NA & & & LC \\
\hline Otospermophilus variegatus (Erxleben, 1777) & IC & NA & & & LC \\
\hline Sciurus aberti Woodhouse, 1853 & $\mathrm{C}$ & NA & $\operatorname{Pr}$ & & LC \\
\hline Sciurus alleni Nelson, 1898 & $\mathrm{C}$ & $M X$ & & & LC \\
\hline Sciurus arizonensis Coues, 1867 & $\mathrm{C}$ & NA & A & & DD \\
\hline Sciurus aureogaster F. Cuvier, 1829 & $\mathrm{C}$ & MA & & & LC \\
\hline Sciurus colliaei Richardson, 1839 & $\mathrm{C}$ & $M X$ & & & LC \\
\hline
\end{tabular}




\begin{tabular}{|c|c|c|c|c|c|}
\hline \multicolumn{6}{|l|}{ Apéndice I. Continuación... } \\
\hline & \multicolumn{2}{|c|}{ Distribución } & \multicolumn{3}{|c|}{ Estado de Conservación } \\
\hline & Ins/Cont & Continente & México & CITES & UICN \\
\hline Sciurus deppei Peters, 1863 & C & MA & & III & LC \\
\hline Sciurus griseus Ord, 1818 & $\mathrm{C}$ & NA & A & & LC \\
\hline Sciurus nayaritensis J. A. Allen, 1890 & $\mathrm{C}$ & NA & & & LC \\
\hline Sciurus niger Linnaeus, 1758 & $\mathrm{C}$ & NA & & & LC \\
\hline Sciurus oculatus Peters, 1863 & $\mathrm{C}$ & MX & $\operatorname{Pr}$ & & LC \\
\hline Sciurus variegatoides Ogilby, 1839 & C & MA & $\operatorname{Pr}$ & & LC \\
\hline Sciurus yucatanensis J. A. Allen, 1877 & C & MA & & & LC \\
\hline Tamiasciurus mearnsi Townsend, 1897 & C & $M X$ & A & & EN \\
\hline Xerospermophilus perotensis (Merriam, 1893) & C & $M X$ & A & & EN \\
\hline Xerospermophilus spilosoma (Bennett, 1833) & $\mathrm{C}$ & NA & & & LC \\
\hline Xerospermophilus tereticaudus (Baird, 1858) & IC & NA & & & LC \\
\hline \multicolumn{6}{|l|}{ FAMILIA CASTORIDAE } \\
\hline Castor canadensis Kuhl, 1820 & C & NA & $\mathrm{P}$ & & LC \\
\hline \multicolumn{6}{|l|}{ FAMILIA HETEROMYIDAE } \\
\hline \multicolumn{6}{|l|}{ SUBFAMILIA DIPODOMYINAE } \\
\hline Dipodomys compactus True, 1889 & IC & NA & & & LC \\
\hline Dipodomys deserti Stephens, 1887 & $\mathrm{C}$ & NA & & & LC \\
\hline Dipodomys gravipes Huey, 1925 & C & $M X$ & EX & & $\mathrm{CR}$ \\
\hline Dipodomys insularis Merriam, 1907 & 1 & $M X$ & $\mathrm{P}$ & & CR \\
\hline Dipodomys merriami Mearns, 1890 & IC & NA & * & & LC \\
\hline Dipodomys nelsoni Merriam, 1907 & $\mathrm{C}$ & $M X$ & & & LC \\
\hline Dipodomys ordii Woodhouse, 1853 & $\mathrm{C}$ & NA & & & LC \\
\hline Dipodomys phillipsii Gray, 1841 & $\mathrm{C}$ & $M X$ & $\operatorname{Pr}$ & & LC \\
\hline Dipodomys simulans Merriam, 1904 & C & NA & & & LC \\
\hline Dipodomys spectabilis Merriam, 1890 & $\mathrm{C}$ & NA & & & NT \\
\hline \multicolumn{6}{|l|}{ SUBFAMILIA HETEROMYINAE } \\
\hline Heteromys desmarestianus Gray, 1868 & $\mathrm{C}$ & SA & & & LC \\
\hline Heteromys gaumeri J. A. Allen \& Chapman, 1897 & $\mathrm{C}$ & MA & & & LC \\
\hline Heteromys nelsoni Merriam, 1902 & C & MA & $\operatorname{Pr}$ & & EN \\
\hline Liomys irroratus (Gray, 1868) & $\mathrm{C}$ & NA & & & LC \\
\hline Liomys pictus (Thomas, 1893) & C & MA & & & LC \\
\hline Liomys salvini (Thomas, 1893) & C & MA & & & LC \\
\hline Liomys spectabilis Genoways, 1971 & $\mathrm{C}$ & $M X$ & $\operatorname{Pr}$ & & EN \\
\hline \multicolumn{6}{|l|}{ SUBFAMILIA PEROGNATHINAE } \\
\hline Chaetodipus anthonyi (Osgood, 1900) & 1 & $M X$ & & & \\
\hline Chaetodipus arenarius Merriam, 1894 & IC & $M X$ & * & & LC \\
\hline Chaetodipus artus (Osgood, 1900) & $\mathrm{C}$ & MX & & & LC \\
\hline Chaetodipus baileyi Merriam, 1894 & IC & NA & * & & LC \\
\hline Chaetodipus californicus (Merriam, 1889) & $\mathrm{C}$ & NA & & & LC \\
\hline Chaetodipus dalquesti (Roth, 1976) & C & MX & $\operatorname{Pr}$ & & VU \\
\hline Chaetodipus eremicus (Mearns, 1898) & C & NA & & & LC \\
\hline Chaetodipus fallax (Merriam, 1889) & $\mathrm{C}$ & NA & & & LC \\
\hline
\end{tabular}




\begin{tabular}{|c|c|c|c|c|c|}
\hline \multicolumn{6}{|l|}{ Apéndice I. Continuación... } \\
\hline & \multicolumn{2}{|c|}{ Distribución } & \multicolumn{3}{|c|}{ Estado de Conservación } \\
\hline & Ins/Cont & Continente & México & CITES & UICN \\
\hline Chaetodipus formosus (Merriam, 1889) & C & NA & & & LC \\
\hline Chaetodipus goldmani (Osgood, 1900) & $\mathrm{C}$ & $M X$ & & & NT \\
\hline Chaetodipus hispidus (Baird, 1858) & $\mathrm{C}$ & NA & & & LC \\
\hline Chaetodipus intermedius (Merriam, 1889) & C & NA & * & & LC \\
\hline Chaetodipus lineatus (Dalquest, 1951) & $\mathrm{C}$ & $M X$ & & & DD \\
\hline Chaetodipus nelsoni (Merriam, 1894) & C & NA & & & LC \\
\hline Chaetodipus penicillatus (Woodhouse, 1852) & IC & NA & * & & LC \\
\hline Chaetodipus pernix (J. A. Allen, 1898) & $\mathrm{C}$ & $M X$ & & & LC \\
\hline Chaetodipus rudinoris (Elliot, 1903) & IC & NA & & & LC \\
\hline Chaetodipus spinatus (Merriam, 1889) & IC & NA & $*$ & & LC \\
\hline Perognathus amplus Osgood, 1900 & C & NA & $*$ & & LC \\
\hline Perognathus flavescens Merriam, 1889 & $\mathrm{C}$ & NA & & & LC \\
\hline Perognathus flavus Baird, 1855 & C & NA & & & LC \\
\hline Perognathus longimembris (Coues, 1875) & $\mathrm{C}$ & NA & & & LC \\
\hline Perognathus merriami J. A. Allen, 1892 & C & NA & & & LC \\
\hline \multicolumn{6}{|l|}{ FAMILIA GEOMYIDAE } \\
\hline Cratogeomys castanops (Baird, 1852) & C & NA & & & LC \\
\hline Cratogeomys fulvescens Merriam, 1895 & C & $M X$ & & & LC \\
\hline Cratogeomys fumosus (Merriam, 1892) & $\mathrm{C}$ & $M X$ & A & & LC \\
\hline Cratogeomys goldmani Merriam, 1895 & C & $M X$ & & & LC \\
\hline Cratogeomys merriami (Thomas, 1893) & $\mathrm{C}$ & $M X$ & & & LC \\
\hline Cratogeomys perotensis Merriam, 1895 & $\mathrm{C}$ & $M X$ & & & LC \\
\hline Cratogeomys planiceps (Merriam, 1895) & $\mathrm{C}$ & MX & & & LC \\
\hline Geomys arenarius Merriam, 1895 & C & NA & & & NT \\
\hline Geomys personatus True, 1889 & $\mathrm{C}$ & NA & A & & LC \\
\hline Geomys tropicalis Goldman, 1915 & C & $M X$ & $\mathrm{~A}$ & & $\mathrm{CR}$ \\
\hline Orthogeomys cuniculus Elliot, 1905 & $\mathrm{C}$ & $M X$ & A & & $\mathrm{DD}$ \\
\hline Orthogeomys grandis (Thomas, 1893) & $\mathrm{C}$ & MA & & & LC \\
\hline Orthogeomys hispidus (Le Conte, 1852) & C & MA & & & LC \\
\hline Orthogeomys lanius (Elliot, 1905) & $\mathrm{C}$ & MX & A & & CR \\
\hline Pappogeomys bulleri (Thomas, 1892) & C & MX & & & LC \\
\hline Thomomys atrovarius J. A. Allen, 1898 & C & $M X$ & & & \\
\hline Thomomys bottae (Eydoux \& Gervais, 1836) & IC & NA & & & LC \\
\hline Thomomys nayarensis Mathis et al., b (en prensa) & C & $M X$ & & & \\
\hline Thomomys sheldoni Bailey, 1915 & $\mathrm{C}$ & $M X$ & & & \\
\hline Thomomys umbrinus (Richardson, 1829) & $\mathrm{C}$ & NA & & & LC \\
\hline Zygogeomys trichopus Merriam, 1895 & $\mathrm{C}$ & $M X$ & $\mathrm{P}$ & & EN \\
\hline \multicolumn{6}{|l|}{ FAMILIA MURIDAE } \\
\hline \multicolumn{6}{|l|}{ SUBFAMILIA ARVICOLINAE } \\
\hline Microtus californicus (Peale, 1848) & C & NA & $\mathrm{P}$ & & LC \\
\hline Microtus guatemalensis Merriam, 1898 & $\mathrm{C}$ & MA & A & & NT \\
\hline Microtus mexicanus (Saussure, 1861) & $\mathrm{C}$ & NA & & & LC \\
\hline
\end{tabular}




\begin{tabular}{|c|c|c|c|c|c|}
\hline \multicolumn{6}{|l|}{ Apéndice I. Continuación... } \\
\hline & \multicolumn{2}{|c|}{ Distribución } & \multicolumn{3}{|c|}{ Estado de Conservación } \\
\hline & Ins/Cont & Continente & México & CITES & UICN \\
\hline Microtus oaxacensis Goodwin, 1966 & C & MX & A & & EN \\
\hline Microtus pennsy/vanicus (Ord, 1815) & $\mathrm{C}$ & NA & $\mathrm{P}$ & & LC \\
\hline Microtus quasiater (Coues, 1874) & $\mathrm{C}$ & $M X$ & $\operatorname{Pr}$ & & NT \\
\hline Microtus umbrosus Merriam, 1898 & $\mathrm{C}$ & MX & $\operatorname{Pr}$ & & EN \\
\hline Ondatra zibethicus (Linnaeus, 1766) & $\mathrm{C}$ & NA & A & & LC \\
\hline \multicolumn{6}{|l|}{ SUBFAMILIA NEOTOMINAE } \\
\hline Baiomys musculus (Merriam, 1892) & $\mathrm{C}$ & MA & & & LC \\
\hline Baiomys taylori (Thomas, 1887) & C & NA & & & LC \\
\hline Habromys chinanteco (Robertson \& Musser, 1976) & C & $M X$ & & & CR \\
\hline Habromys delicatulus Carleton et al., 2002 & C & $M X$ & & & CR \\
\hline Habromys ixtlani (Goodwin, 1964) & $\mathrm{C}$ & $M X$ & & & CR \\
\hline Habromys lepturus (Merriam, 1898) & $\mathrm{C}$ & MX & & & CR \\
\hline Habromys lophurus (Osgood, 1904) & C & MA & & & NT \\
\hline Habromys schmidlyi Romo-Vázquez et al., 2005 & $\mathrm{C}$ & MX & & & $\mathrm{CR}$ \\
\hline Habromys simulatus (Osgood, 1904) & C & $M X$ & $\mathrm{P}$ & & EN \\
\hline Hodomys alleni (Merriam, 1892) & C & MX & & & LC \\
\hline Megadontomys cryophilus (Musser, 1964) & C & $M X$ & $\mathrm{~A}$ & & EN \\
\hline Megadontomys nelsoni (Merriam, 1898) & C & $M X$ & A & & EN \\
\hline Megadontomys thomasi (Merriam, 1898) & C & $M X$ & $\operatorname{Pr}$ & & EN \\
\hline Nelsonia goldmani Merriam, 1903 & C & $M X$ & $\operatorname{Pr}$ & & EN \\
\hline Nelsonia neotomodon Merriam, 1897 & $\mathrm{C}$ & $M X$ & $\operatorname{Pr}$ & & NT \\
\hline Neotoma albigula Hartley, 1894 & IC & NA & $*$ & & LC \\
\hline Neotoma angustapalata Baker, 1951 & $\mathrm{C}$ & $M X$ & & & EN \\
\hline Neotoma bryanti Merriam, 1887 & 1 & $M X$ & $\mathrm{~A}$ & & EN \\
\hline Neotoma devia Goldman, 1927 & C & NA & & & LC \\
\hline Neotoma goldmani Merriam, 1903 & $\mathrm{C}$ & $M X$ & & & LC \\
\hline Neotoma insularis Townsend, 1912 & 1 & $M X$ & & & \\
\hline Neotoma isthmica Goldman 1904 & $\mathrm{C}$ & $M X$ & & & \\
\hline Neotoma lepida Thomas, 1893 & IC & NA & * & & LC \\
\hline Neotoma leucodon Merrian, 1894 & $\mathrm{C}$ & NA & & & LC \\
\hline Neotoma macrotis Thomas, 1893 & C & NA & & & LC \\
\hline Neotoma mexicana Baird, 1855 & $\mathrm{C}$ & NA & & & LC \\
\hline Neotoma micropus Baird, 1855 & $\mathrm{C}$ & NA & & & LC \\
\hline Neotoma nelsoni Goldman, 1905 & C & $M X$ & & & $\mathrm{CR}$ \\
\hline Neotoma palatina Goldman, 1905 & $\mathrm{C}$ & $M X$ & & & VU \\
\hline Neotoma phenax (Merriam, 1903) & $\mathrm{C}$ & MX & $\operatorname{Pr}$ & & NT \\
\hline Neotoma picta Goldman 1904 & $\mathrm{C}$ & $M X$ & & & \\
\hline Neotomodon alstoni Merriam, 1898 & C & $M X$ & & & LC \\
\hline Nyctomys sumichrasti (Saussure, 1860) & $\mathrm{C}$ & MA & & & LC \\
\hline Oligoryzomys fulvescens (Saussure, 1860) & C & SA & & & $\mathrm{LCL}$ \\
\hline Onychomys arenicola Mearns, 1896 & $\mathrm{C}$ & NA & & & LC \\
\hline Onychomys leucogaster (Wied-Neuwied, 1841) & $\mathrm{C}$ & NA & & & LC \\
\hline
\end{tabular}




\begin{tabular}{|c|c|c|c|c|c|}
\hline \multicolumn{6}{|l|}{ Apéndice I. Continuación... } \\
\hline & \multicolumn{2}{|c|}{ Distribución } & \multicolumn{3}{|c|}{ Estado de Conservación } \\
\hline & Ins/Cont & Continente & México & CITES & UICN \\
\hline Onychomys torridus (Coues, 1874) & $\mathrm{C}$ & $\mathrm{NA}$ & & & LC \\
\hline Oryzomys albiventer Merriam, 1901 & $\mathrm{C}$ & $M X$ & & & \\
\hline Oryzomys alfaroi (J. A. Allen, 1891) & $\mathrm{C}$ & SA & & & LC \\
\hline Oryzomys chapmani Thomas, 1898 & $\mathrm{C}$ & $M X$ & & & LC \\
\hline Oryzomys couesi (Alston, 1877) & IC & AM & & & LC \\
\hline Oryzomys melanotis Thomas, 1893 & $\mathrm{C}$ & $M X$ & & & LC \\
\hline Oryzomys mexicanus Merriam, 1897 & $\mathrm{C}$ & MX & & & \\
\hline Oryzomys nelsoni Merriam, 1898 & 1 & MX & EX & & EX \\
\hline Oryzomys rhabdops Merriam, 1901 & $\mathrm{C}$ & MA & & & VU \\
\hline Oryzomys rostratus Merriam, 1901 & $\mathrm{C}$ & MA & & & LC \\
\hline Oryzomys saturatior Merriam, 1901 & $\mathrm{C}$ & MA & & & NT \\
\hline Oryzomys texensis J. A. Allen, 1894 & $\mathrm{C}$ & NA & & & \\
\hline Osgoodomys banderanus (J. A. Allen, 1897) & $\mathrm{C}$ & $M X$ & & & LC \\
\hline Otonyctomys hatti Anthony, 1932 & C & MA & A & & LC \\
\hline Ototylomys phyllotis Merriam, 1901 & C & MA & & & LC \\
\hline Peromyscus aztecus (Saussure, 1860) & $\mathrm{C}$ & MA & & & LC \\
\hline Peromyscus beatae Thomas, 1903 & C & $M X$ & & & LC \\
\hline Peromyscus boylii (Baird, 1855) & IC & NA & * & & LC \\
\hline Peromyscus bullatus Osgood, 1904 & $\mathrm{C}$ & $M X$ & $\operatorname{Pr}$ & & $\mathrm{CR}$ \\
\hline Peromyscus californicus (Gambel, 1848) & $\mathrm{C}$ & NA & & & LC \\
\hline Peromyscus caniceps Burt, 1932 & 1 & $M X$ & $\operatorname{Pr}$ & & $\mathrm{CR}$ \\
\hline Peromyscus crinitus (Merriam, 1891) & IC & $M X$ & $*$ & & LC \\
\hline Peromyscus dickeyi Burt, 1932 & 1 & $M X$ & $\operatorname{Pr}$ & & $\mathrm{CR}$ \\
\hline Peromyscus difficilis (J. A. Allen, 1891) & $\mathrm{C}$ & $M X$ & & & LC \\
\hline Peromyscus eremicus (Baird, 1858) & IC & NA & $*$ & & LC \\
\hline Peromyscus eva Thomas, 1898 & $\mathrm{C}$ & $M X$ & * & & LC \\
\hline Peromyscus fraterculus (Miller, 1892) & $\mathrm{C}$ & NA & & & LC \\
\hline Peromyscus furvus J. A. Allen \& Chapman, 1897 & C & $M X$ & & & DD \\
\hline Peromyscus gratus Merriam, 1898 & $\mathrm{C}$ & NA & & & LC \\
\hline Peromyscus guardia Townsend, 1912 & 1 & $M X$ & $\mathrm{P}$ & & $\mathrm{CR}$ \\
\hline Peromyscus guatemalensis Merriam, 1898 & C & MA & & & LC \\
\hline Peromyscus gymnotis Thomas, 1894 & $\mathrm{C}$ & MA & & & LC \\
\hline Peromyscus hooperi Lee \& Schmidly, 1977 & $\mathrm{C}$ & $M X$ & & & LC \\
\hline Peromyscus hylocetes Merriam, 1898 & C & $M X$ & & & LC \\
\hline Peromyscus interparietalis Burt, 1932 & I & $M X$ & A & & $\mathrm{CR}$ \\
\hline Peromyscus leucopus (Rafinesque, 1818) & IC & NA & * & & LC \\
\hline Peromyscus levipes Merriam, 1898 & $\mathrm{C}$ & $M X$ & & & LC \\
\hline Peromyscus madrensis Merriam, 1898 & 1 & $M X$ & A & & EN \\
\hline Peromyscus maniculatus (Wagner, 1845) & IC & NA & * & & LC \\
\hline Peromyscus megalops Merriam, 1898 & $\mathrm{C}$ & $M X$ & & & LC \\
\hline Peromyscus mekisturus Merriam, 1898 & C & $M X$ & A & & $\mathrm{CR}$ \\
\hline Peromyscus melanocarpus Osgood, 1904 & $\mathrm{C}$ & $M X$ & & & EN \\
\hline
\end{tabular}




\begin{tabular}{|c|c|c|c|c|c|}
\hline \multicolumn{6}{|l|}{ Apéndice I. Continuación... } \\
\hline & \multicolumn{2}{|c|}{ Distribución } & \multicolumn{3}{|c|}{ Estado de Conservación } \\
\hline & Ins/Cont & Continente & México & CITES & UICN \\
\hline Peromyscus melanophrys (Coues, 1874) & C & $M X$ & & & LC \\
\hline Peromyscus melanotis J. A. Allen \& Chapman, 1897 & C & NA & & & LC \\
\hline Peromyscus melanurus Osgood, 1909 & $\mathrm{C}$ & MX & & & EN \\
\hline Peromyscus merriami Mearns, 1896 & C & NA & & & LC \\
\hline Peromyscus mexicanus (Saussure, 1860) & $\mathrm{C}$ & MA & & & LC \\
\hline Peromyscus nasutus (J. A. Allen, 1891) & $\mathrm{C}$ & NA & & & LC \\
\hline Peromyscus oaxacensis Merriam 1898 & $\mathrm{C}$ & MA & & & \\
\hline Peromyscus ochraventer Baker, 1951 & C & MX & & & EN \\
\hline Peromyscus pectoralis Osgood, 1904 & $\mathrm{C}$ & NA & & & LC \\
\hline Peromyscus pembertoni Burt, 1932 & 1 & $M X$ & EX & & EX \\
\hline Peromyscus perfulvus Osgood, 1945 & $\mathrm{C}$ & $M X$ & & & LC \\
\hline Peromyscus polius Osgood, 1904 & $\mathrm{C}$ & MX & & & NT \\
\hline Peromyscus pseudocrinitus Burt, 1932 & 1 & $M X$ & A & & CR \\
\hline Peromyscus sagax Elliot, 1903 & $\mathrm{C}$ & $M X$ & & & DD \\
\hline Peromyscus schmidlyi Bradley et al., 2004 & $\mathrm{C}$ & $\mathrm{MX}$ & & & LC \\
\hline Peromyscus sejugis Burt, 1932 & 1 & $M X$ & A & & EN \\
\hline Peromyscus simulus Osgood, 1904 & $\mathrm{C}$ & MX & & & $\mathrm{VU}$ \\
\hline Peromyscus slevini Mailliard, 1924 & 1 & $M X$ & A & & CR \\
\hline Peromyscus spicilegus J. A. Allen, 1897 & C & $M X$ & & & LC \\
\hline Peromyscus stephani Townsend, 1912 & 1 & $M X$ & A & & CR \\
\hline Peromyscus truei (Shufeldt, 1885) & $\mathrm{C}$ & NA & & & LC \\
\hline Peromyscus winkelmanni Carleton, 1977 & C & $M X$ & $\operatorname{Pr}$ & & EN \\
\hline Peromyscus yucatanicus J. A. Allen \& Chapman, 1897 & $\mathrm{C}$ & $M X$ & & & LC \\
\hline Peromyscus zarhynchus Merriam, 1898 & $\mathrm{C}$ & $\mathrm{MX}$ & $\operatorname{Pr}$ & & $\mathrm{VU}$ \\
\hline Reithrodontomys bakeri Bradley et al., 2004 & $\mathrm{C}$ & $M X$ & & & EN \\
\hline Reithrodontomys burti Benson, 1939 & $\mathrm{C}$ & MX & & & DD \\
\hline Reithrodontomys chrysopsis Merriam, 1900 & $\mathrm{C}$ & $M X$ & & & LC \\
\hline Reithrodontomys fulvescens J. A. Allen, 1894 & $\mathrm{C}$ & NA & & & LC \\
\hline Reithrodontomys gracilis J. A. Allen \& Chapman, 1897 & IC & MA & $*$ & & LC \\
\hline Reithrodontomys hirsutus Merriam, 1901 & $\mathrm{C}$ & $M X$ & & & $\mathrm{VU}$ \\
\hline Reithrodontomys megalotis (Baird, 1858) & $\mathrm{C}$ & NA & & & LC \\
\hline Reithrodontomys mexicanus (Saussure, 1860) & $\mathrm{C}$ & MA & & & LC \\
\hline Reithrodontomys microdon Merriam, 1901 & $\mathrm{C}$ & MA & A & & LC \\
\hline Reithrodontomys montanus (Baird, 1855) & $\mathrm{C}$ & NA & & & LC \\
\hline Reithrodontomys spectabilis Jones \& Lawlor, 1965 & 1 & MX & A & & CR \\
\hline Reithrodontomys sumichrasti (Saussure, 1861) & $\mathrm{C}$ & MA & & & LC \\
\hline Reithrodontomys tenuirostris Merriam, 1901 & $\mathrm{C}$ & MA & & & $\mathrm{VU}$ \\
\hline Reithrodontomys zacatecae Merriam, 1901 & $\mathrm{C}$ & MX & & & LC \\
\hline Rheomys mexicanus Goodwin, 1959 & $\mathrm{C}$ & $M X$ & $\operatorname{Pr}$ & & EN \\
\hline Rheomys thomasi Dickey, 1928 & $\mathrm{C}$ & MA & $\operatorname{Pr}$ & & NT \\
\hline Scotinomys teguina (Alston, 1877) & $\mathrm{C}$ & MA & $*$ & & LC \\
\hline Sigmodon alleni Bailey, 1902 & $\mathrm{C}$ & $\mathrm{MX}$ & & & $\mathrm{VU}$ \\
\hline
\end{tabular}




\begin{tabular}{|c|c|c|c|c|c|}
\hline \multicolumn{6}{|l|}{ Apéndice I. Continuación... } \\
\hline & \multicolumn{2}{|c|}{ Distribución } & \multicolumn{3}{|c|}{ Estado de Conservación } \\
\hline & Ins/Cont & Continente & México & CITES & UICN \\
\hline Sigmodon arizonae Mearns, 1890 & C & NA & & & LC \\
\hline Sigmodon fulviventer J. A. Allen, 1889 & $\mathrm{C}$ & NA & & & LC \\
\hline Sigmodon hirsutus (Burmeister, 1854) & $\mathrm{C}$ & SA & & & LC \\
\hline Sigmodon hispidus Say \& Ord, 1825 & C & NA & & & LC \\
\hline Sigmodon leucotis Bailey, 1902 & $\mathrm{C}$ & MX & & & LC \\
\hline Sigmodon mascotensis J. A. Allen, 1897 & C & $M X$ & & & LC \\
\hline Sigmodon ochrognathus Bailey, 1902 & C & NA & & & LC \\
\hline Sigmodon planifrons Nelson \& Goldman, 1933 & C & $M X$ & & & EN \\
\hline Sigmodon toltecus (Saussure, 1860) & C & MA & & & LC \\
\hline Sigmodon zanjonensis Goodwin, 1932 & C & MA & & & \\
\hline Tylomys bullaris Merriam, 1901 & $\mathrm{C}$ & $M X$ & A & & CR \\
\hline Tylomys nudicaudus Peters, 1866 & $\mathrm{C}$ & MA & & & LC \\
\hline Tylomys tumbalensis Merriam, 1901 & C & $M X$ & $\operatorname{Pr}$ & & $\mathrm{CR}$ \\
\hline Xenomys nelsoni Merriam, 1892 & $\mathrm{C}$ & $M X$ & A & & EN \\
\hline \multicolumn{6}{|l|}{ FAMILIA ERETHIZONTIDAE } \\
\hline \multicolumn{6}{|l|}{ SUBFAMILIA ERETHIZONTINAE } \\
\hline Erethizon dorsatum (Linnaeus, 1758) & $\mathrm{C}$ & NA & $\mathrm{P}$ & & LC \\
\hline Sphiggurus mexicanus (Kerr, 1792) & C & MA & $\mathrm{A}$ & III & LC \\
\hline \multicolumn{6}{|l|}{ FAMILIA DASYPROCTIDAE } \\
\hline Dasyprocta mexicana Saussure, 1860 & C & $M X$ & & & $\mathrm{CR}$ \\
\hline Dasyprocta punctata Gray, 1842 & IC & SA & & III & LC \\
\hline \multicolumn{6}{|l|}{ FAMILIA CUNICULIDAE } \\
\hline Cuniculus paca (Linnaeus, 1776) & IC & SA & & III & LC \\
\hline \multicolumn{6}{|l|}{ ORDEN SORICOMORPHA } \\
\hline \multicolumn{6}{|l|}{ FAMILIA SORICIDAE } \\
\hline \multicolumn{6}{|l|}{ SUBFAMILIA SORICINAE } \\
\hline Cryptotis alticola (Merriam, 1895) & $\mathrm{C}$ & $M X$ & $\operatorname{Pr}$ & & DD \\
\hline Cryptotis goldmani (Merriam, 1895) & C & $M X$ & $\operatorname{Pr}$ & & LC \\
\hline Cryptotis goodwini Jackson, 1933 & $\mathrm{C}$ & MA & & & LC \\
\hline Cryptotis griseoventris Jackson, 1933 & C & MA & & & VU \\
\hline Cryptotis magna (Merriam, 1895) & $\mathrm{C}$ & $M X$ & $\operatorname{Pr}$ & & $\mathrm{VU}$ \\
\hline Cryptotis mayensis (Merriam, 1901) & $\mathrm{C}$ & MA & $\operatorname{Pr}$ & & LC \\
\hline Cryptotis merriami Choate, 1970 & C & MA & & & LC \\
\hline Cryptotis mexicana (Coues, 1877) & $\mathrm{C}$ & $M X$ & & & LC \\
\hline Cryptotis nelsoni (Merriam, 1895) & $\mathrm{C}$ & $M X$ & $\operatorname{Pr}$ & & CR \\
\hline Cryptotis obscura (Merriam, 1895) & $\mathrm{C}$ & $M X$ & $\operatorname{Pr}$ & & VU \\
\hline Cryptotis parva (Say, 1823) & C & NA & * & & LC \\
\hline Cryptotis peregrina (Merriam, 1895) & $\mathrm{C}$ & $M X$ & $\operatorname{Pr}$ & & DD \\
\hline Cryptotis phillipsii (Schaldach, 1966) & C & $M X$ & & & VU \\
\hline Cryptotis tropicalis (Merriam, 1895) & C & MA & & & DD \\
\hline Megasorex gigas (Merriam, 1897) & $\mathrm{C}$ & $M X$ & $\mathrm{~A}$ & & LC \\
\hline
\end{tabular}




\begin{tabular}{|c|c|c|c|c|c|}
\hline \multicolumn{6}{|l|}{ Apéndice I. Continuación... } \\
\hline & \multicolumn{2}{|c|}{ Distribución } & \multicolumn{3}{|c|}{ Estado de Conservación } \\
\hline & Ins/Cont & Continente & México & CITES & UICN \\
\hline Notiosorex cockrumi Baker et al., 2003 & $\mathrm{C}$ & NA & & & LC \\
\hline Notiosorex crawfordi (Coues, 1877) & IC & NA & A & & LC \\
\hline Notiosorex evotis (Coues, 1877) & $\mathrm{C}$ & $M X$ & A & & LC \\
\hline Notiosorex villai Carraway \& Timm, 2000 & $\mathrm{C}$ & $M X$ & A & & VU \\
\hline Sorex arizonae Diersing \& Hoffmeister, 1977 & $\mathrm{C}$ & NA & $P$ & & LC \\
\hline Sorex emarginatus Jackson, 1925 & $\mathrm{C}$ & $M X$ & & & LC \\
\hline Sorex ixtlanensis Carraway, 2007 & $\mathrm{C}$ & $M X$ & & & DD \\
\hline Sorex macrodon Merriam, 1895 & $\mathrm{C}$ & $M X$ & A & & $\mathrm{VU}$ \\
\hline Sorex mediopua Carraway, 2007 & $\mathrm{C}$ & $M X$ & & & $\mathrm{CR}$ \\
\hline Sorex milleri Jackson, 1947 & C & $M X$ & $\operatorname{Pr}$ & & VU \\
\hline Sorex monticolus Merriam, 1890 & $\mathrm{C}$ & NA & A & & LC \\
\hline Sorex oreopolus Merriam, 1892 & C & $M X$ & & & LC \\
\hline Sorex orizabae Merriam 1895 & C & $M X$ & & & LC \\
\hline Sorex ornatus Merriam, 1895 & C & NA & * & & LC \\
\hline Sorex saussurei Merriam, 1892 & C & MA & & & LC \\
\hline Sorex sclateri Merriam, 1897 & C & $M X$ & A & & CR \\
\hline Sorex stizodon Merriam, 1895 & C & $M X$ & A & & CR \\
\hline Sorex ventralis Merriam, 1895 & $\mathrm{C}$ & $M X$ & & & LC \\
\hline Sorex veraecrucis Jackson, 1925 & C & $M X$ & $\operatorname{Pr}$ & & LC \\
\hline Sorex veraepacis Alston, 1877 & C & MA & A & & LC \\
\hline \multicolumn{6}{|l|}{ FAMILIA TALPIDAE } \\
\hline \multicolumn{6}{|l|}{ SUBFAMILIA TALPINAE } \\
\hline Scalopus aquaticus (Linnaeus, 1758) & C & NA & $\mathrm{P}$ & & LC \\
\hline Scapanus anthony J. A. Allen, 1893 & $\mathrm{C}$ & $M X$ & $\mathrm{P}$ & & \\
\hline Scapanus latimanus (Bachman, 1842) & C & NA & A & & LC \\
\hline \multicolumn{6}{|l|}{ ORDEN CARNIVORA } \\
\hline \multicolumn{6}{|l|}{ FAMILIA FELIDAE } \\
\hline \multicolumn{6}{|l|}{ SUBFAMILIA FELINAE } \\
\hline Leopardus pardalis (Linnaeus, 1758) & $\mathrm{C}$ & AM & $\mathrm{P}$ & 1 & LC \\
\hline Leopardus wiedii (Schinz, 1821) & $\mathrm{C}$ & SA & $P$ & I & NT \\
\hline Lynx rufus (Schreber, 1777) & $\mathrm{C}$ & NA & & II & LC \\
\hline Puma concolor (Linnaeus, 1771) & C & AM & & II & LC \\
\hline Puma yagouaroundi (Lacépède, 1809) & $\mathrm{C}$ & AM & A & I & LC \\
\hline \multicolumn{6}{|l|}{ SUBFAMILIA PANTHERINAE } \\
\hline Panthera onca (Linnaeus, 1758) & $\mathrm{C}$ & AM & $\mathrm{P}$ & I & NT \\
\hline \multicolumn{6}{|l|}{ FAMILIA CANIDAE } \\
\hline Canis latrans Say, 1823 & IC & AM & & & LC \\
\hline Canis lupus Linnaeus, 1758 & C & NA & EX & II & LC \\
\hline Urocyon cinereoargenteus (Schreber, 1775) & IC & AM & & & LC \\
\hline Vulpes macrotis Merriam, 1888 & $\mathrm{C}$ & NA & A & & LC \\
\hline
\end{tabular}




\begin{tabular}{|c|c|c|c|c|c|}
\hline \multirow[t]{3}{*}{ Apéndice I. Continuación... } & & & & & \\
\hline & \multicolumn{2}{|c|}{ Distribución } & \multicolumn{3}{|c|}{ Estado de Conservación } \\
\hline & Ins/Cont & Continente & México & CITES & UICN \\
\hline \multicolumn{6}{|l|}{ FAMILIA URSIDAE } \\
\hline \multicolumn{6}{|l|}{ SUBFAMILIA URSINAE } \\
\hline Ursus americanus Pallas, 1780 & $\mathrm{C}$ & NA & $\mathrm{P} / \mathrm{Pr}$ & II & LC \\
\hline Ursus arctos Linnaeus, 1758 & C & NA & EX & II & LC \\
\hline \multicolumn{6}{|l|}{ FAMILIA OTARIIDAE } \\
\hline Arctocephalus galapageoensis Heller, 1904 & M & SA & & II & EN \\
\hline Arctocephalus townsendi Merriam, 1897 & M & NA & $\mathrm{P}$ & 1 & NT \\
\hline Callorhinus ursinus (Linnaeus, 1758) & M & NA & & & $\mathrm{VU}$ \\
\hline Zalophus californianus (Lesson, 1828) & M & NA & $\operatorname{Pr}$ & & LC \\
\hline Zalophus wollebaeki (Sivertsen, 1953) & M & SA & & & EN \\
\hline \multicolumn{6}{|l|}{ FAMILIA PHOCIDAE } \\
\hline Mirounga angustirostris (Gill, 1866) & M & NA & A & & LC \\
\hline Monachus tropicalis (Gray, 1850) & M & MA & EX & I & EX \\
\hline Phoca vitulina Linnaeus, 1758 & M & NA & $\operatorname{Pr}$ & & LC \\
\hline \multicolumn{6}{|l|}{ FAMILIA MUSTELIDAE } \\
\hline \multicolumn{6}{|l|}{ SUBFAMILIA LUTRINAE } \\
\hline Enhydra lutris (Linnaeus, 1758) & M & NA & $P$ & I & EN \\
\hline Lontra canadensis (Schreber, 1777) & $\mathrm{C}$ & NA & & II & LC \\
\hline Lontra longicaudis (Olfers, 1818) & C & SA & A & I & DD \\
\hline \multicolumn{6}{|l|}{ SUBFAMILIA MUSTELINAE } \\
\hline Eira barbara (Linnaeus, 1758) & $\mathrm{C}$ & SA & $P$ & III & LC \\
\hline Galictis vittata (Schreber, 1776) & C & SA & A & III & LC \\
\hline Mustela frenata Lichtenstein, 1831 & C & AM & & & LC \\
\hline Mustela nigripes (Audubon \& Bachman, 1851) & $\mathrm{C}$ & NA & & I & EN \\
\hline \multicolumn{6}{|l|}{ SUBFAMILIA TAXIDIINAE } \\
\hline Taxidea taxus (Schreber, 1777) & $\mathrm{C}$ & NA & A & & LC \\
\hline \multicolumn{6}{|l|}{ FAMILIA MEPHITINAE } \\
\hline Conepatus leuconotus (Lichtenstein, 1832) & C & NA & & & LC \\
\hline Conepatus semistriatus (Boddaert, 1785) & $\mathrm{C}$ & SA & * & & LC \\
\hline Mephitis macroura Lichtenstein, 1832 & C & NA & & & LC \\
\hline Mephitis mephitis (Schreber, 1776) & C & NA & & & LC \\
\hline Spilogale angustifrons Howell, 1902 & C & MA & & & LC \\
\hline Spilogale gracilis Merriam, 1890 & $\mathrm{C}$ & NA & & & LC \\
\hline Spilogale pygmaea Thomas, 1898 & C & $M X$ & A & & VU \\
\hline \multicolumn{6}{|l|}{ FAMILIA PROCYONIDAE } \\
\hline \multicolumn{6}{|l|}{ SUBFAMILIA POTOSINAE } \\
\hline Potos flavus (Schreber, 1774) & $\mathrm{C}$ & SA & $\operatorname{Pr}$ & III & LC \\
\hline \multicolumn{6}{|l|}{ SUBFAMILIA PROCYONINAE } \\
\hline Bassariscus astutus (Lichtenstein, 1830) & IC & NA & * & & LC \\
\hline Bassariscus sumichrasti (Saussure, 1860) & $\mathrm{C}$ & MA & $\operatorname{Pr}$ & III & LC \\
\hline Nasua narica (Linnaeus, 1776) & C & AM & & III & LC \\
\hline
\end{tabular}




\begin{tabular}{|c|c|c|c|c|c|}
\hline \multicolumn{6}{|l|}{ Apéndice I. Continuación... } \\
\hline & \multicolumn{2}{|c|}{ Distribución } & \multicolumn{3}{|c|}{ Estado de Conservación } \\
\hline & Ins/Cont & Continente & México & CITES & UICN \\
\hline Nasua nelsoni (Merriam, 1901) & I & $M X$ & A & & \\
\hline Procyon lotor (Linnaeus, 1758) & $\mathrm{C}$ & AM & * & & LC \\
\hline Procyon pygmaeus Merriam, 1901 & I & MX & $\mathrm{P}$ & & $\mathrm{CR}$ \\
\hline \multicolumn{6}{|l|}{ ORDEN PERISSODACTYLA } \\
\hline \multicolumn{6}{|l|}{ FAMILIA TAPIRIDAE } \\
\hline Tapirus bairdii (Gill, 1865) & $\mathrm{C}$ & SA & $\mathrm{P}$ & I & EN \\
\hline \multicolumn{6}{|l|}{ ORDEN ARTIODACTYLA } \\
\hline \multicolumn{6}{|l|}{ FAMILIA TAYASSUIDAE } \\
\hline Pecari tajacu (Linnaeus, 1758) & IC & SA & & II & LC \\
\hline Tayassu pecari (Link, 1795) & C & AM & $\mathrm{P}$ & II & $\mathrm{VU}$ \\
\hline \multicolumn{6}{|l|}{ FAMILIA CERVIDAE } \\
\hline \multicolumn{6}{|l|}{ SUBFAMILIA CERVINAE } \\
\hline Cervus canadensis Erxleben, 1777 & C & NA & EX & & \\
\hline \multicolumn{6}{|l|}{ SUBFAMILIA ODOCOILEINAE } \\
\hline Mazama americana (Erxleben, 1777) & $\mathrm{C}$ & SA & & & $\mathrm{DD}$ \\
\hline Mazama pandora Merriam, 1901 & C & MA & & & VU \\
\hline Odocoileus hemionus (Rafinesque, 1817) & IC & NA & $*$ & & LC \\
\hline Odocoileus virginianus (Zimmermann, 1780) & IC & AM & & III & LC \\
\hline \multicolumn{6}{|l|}{ FAMILIA ANTILOCAPRIDAE } \\
\hline Antilocapra americana (Ord, 1815) & $\mathrm{C}$ & NA & $\mathrm{P}$ & 1 & LC \\
\hline \multicolumn{6}{|l|}{ FAMILIA BOVIDAE } \\
\hline \multicolumn{6}{|l|}{ SUBFAMILIA BOVINAE } \\
\hline Bison bison (Linnaeus, 1758) & C & NA & $\mathrm{P}$ & II & NT \\
\hline \multicolumn{6}{|l|}{ SUBFAMILIA CAPRINAE } \\
\hline Ovis canadensis Shaw, 1804 & C & NA & $\operatorname{Pr}$ & II & LC \\
\hline \multicolumn{6}{|l|}{ ORDEN CETACEA } \\
\hline \multicolumn{6}{|l|}{ FAMILIA BALAENIDAE } \\
\hline Eubalaena japonica (Lacépède, 1818) & M & NA & $\mathrm{P}$ & 1 & EN \\
\hline \multicolumn{6}{|l|}{ FAMILIA BALAENOPTERIDAE } \\
\hline Balaenoptera acutorostrata Lacépède, 1804 & M & AM & $\operatorname{Pr}$ & I & LC \\
\hline Balaenoptera borealis Lesson, 1828 & M & AM & $\operatorname{Pr}$ & 1 & EN \\
\hline Balaenoptera edeni Anderson, 1879 & M & AM & $\operatorname{Pr}$ & 1 & DD \\
\hline Balaenoptera musculus (Linnaeus, 1758) & M & AM & $\operatorname{Pr}$ & 1 & EN \\
\hline Balaenoptera physalus (Linnaeus, 1758) & M & AM & $\operatorname{Pr}$ & 1 & EN \\
\hline Megaptera novaeangliae (Borowski, 1781) & M & AM & $\operatorname{Pr}$ & I & LC \\
\hline \multicolumn{6}{|l|}{ FAMILIA ESCHRICHTIDAE } \\
\hline Eschrichtius robustus (Lilljeborg, 1861) & M & NA & $\operatorname{Pr}$ & 1 & LC \\
\hline \multicolumn{6}{|l|}{ SUBORDEN ODONTOCETI } \\
\hline \multicolumn{6}{|l|}{ FAMILIA PHYSETERIDAE } \\
\hline Kogia breviceps (De Blainville, 1838) & M & AM & $\operatorname{Pr}$ & II & DD \\
\hline Kogia sima (Owen, 1866) & M & AM & $\operatorname{Pr}$ & II & DD \\
\hline
\end{tabular}




\begin{tabular}{|c|c|c|c|c|c|}
\hline \multicolumn{6}{|l|}{ Apéndice I. Continuación... } \\
\hline & \multicolumn{2}{|c|}{ Distribución } & \multicolumn{3}{|c|}{ Estado de Conservación } \\
\hline & Ins/Cont & Continente & México & CITES & UICN \\
\hline Physeter macrocephalus Linnaeus, 1758 & M & AM & $\operatorname{Pr}$ & I & $\mathrm{VU}$ \\
\hline \multicolumn{6}{|l|}{ FAMILIA ZIPHIIDAE } \\
\hline Berardius bairdii Stejneger, 1883 & M & NA & $\operatorname{Pr}$ & 1 & DD \\
\hline Indopacetus pacificus (Longman,1926) & M & AM & & II & DD \\
\hline Mesoplodon carlhubbsi Moore, 1963 & M & NA & & II & DD \\
\hline Mesoplodon densirostris (De Blainville, 1817) & M & AM & $\operatorname{Pr}$ & II & DD \\
\hline Mesoplodon europaeus (Gervais, 1855) & M & NA & $\operatorname{Pr}$ & II & DD \\
\hline Mesoplodon ginkgodens Nishiwaki \& Kamiya, 1958 & M & AM & $\operatorname{Pr}$ & II & DD \\
\hline Mesoplodon perrini Dalebout et al., 2002 & M & NA & & II & DD \\
\hline Mesoplodon peruvianus Reyes et al., 1991 & M & AM & $\operatorname{Pr}$ & II & DD \\
\hline Ziphius cavirostris G. Cuvier, 1823 & M & AM & $\operatorname{Pr}$ & II & LC \\
\hline \multicolumn{6}{|l|}{ FAMILIA DELPHINIDAE } \\
\hline Delphinus capensis Gray, 1828 & M & AM & $\operatorname{Pr}$ & II & DD \\
\hline Delphinus delphis Linnaeus, 1758 & M & AM & $\operatorname{Pr}$ & II & LC \\
\hline Feresa attenuata Gray, 1875 & M & AM & $\operatorname{Pr}$ & II & DD \\
\hline Globicephala macrorhynchus Gray, 1846 & M & AM & $\operatorname{Pr}$ & II & DD \\
\hline Grampus griseus (G. Cuvier, 1812) & M & AM & $\operatorname{Pr}$ & II & LC \\
\hline Lagenodelphis hosei Fraser, 1956 & M & AM & $\operatorname{Pr}$ & II & LC \\
\hline Lagenorhynchus obliquidens Gill, 1865 & M & NA & $\operatorname{Pr}$ & II & LC \\
\hline Lissodelphis borealis (Peale, 1848) & M & NA & $\operatorname{Pr}$ & II & LC \\
\hline Orcinus orca (Linnaeus, 1758) & M & AM & $\operatorname{Pr}$ & II & DD \\
\hline Peponocephala electra (Gray, 1846) & M & AM & $\operatorname{Pr}$ & II & LC \\
\hline Pseudorca crassidens (Owen, 1846) & M & AM & $\operatorname{Pr}$ & II & DD \\
\hline Stenella attenuata (Gray, 1846) & M & AM & $\operatorname{Pr}$ & II & LC \\
\hline Stenella clymene (Gray, 1846) & M & AM & $\operatorname{Pr}$ & II & DD \\
\hline Stenella coeruleoalba (Meyen, 1833) & M & AM & $\operatorname{Pr}$ & II & LC \\
\hline Stenella frontalis (G. Cuvier, 1829) & M & AM & $\operatorname{Pr}$ & II & DD \\
\hline Stenella longirostris (Gray, 1828) & M & AM & $\operatorname{Pr}$ & II & DD \\
\hline Steno bredanensis (G. Cuvier in Lesson, 1828) & M & AM & $\operatorname{Pr}$ & II & LC \\
\hline Tursiops truncatus (Montagu, 1821) & M & AM & $\operatorname{Pr}$ & II & LC \\
\hline \multicolumn{6}{|l|}{ FAMILIA PHOCOENIDAE } \\
\hline Phocoena sinus Norris \& McFarland, 1958 & M & $M X$ & $\mathrm{P}$ & 1 & CR \\
\hline Phocoenoides dalli (True, 1885) & M & NA & $\operatorname{Pr}$ & II & LC \\
\hline \multicolumn{6}{|l|}{ ORDEN CHIROPTERA } \\
\hline \multicolumn{6}{|l|}{ FAMILIA EMBALLONURIDAE } \\
\hline \multicolumn{6}{|l|}{ SUBFAMILIA EMBALLONURINAE } \\
\hline Balantiopteryx io Thomas, 1904 & $\mathrm{C}$ & MA & & & $\mathrm{VU}$ \\
\hline Balantiopteryx plicata Peters, 1867 & IC & SA & & & LC \\
\hline Centronycteris centralis Thomas, 1912 & $\mathrm{C}$ & SA & $\operatorname{Pr}$ & & LC \\
\hline Diclidurus albus Wied- Neuwied, 1820 & $\mathrm{C}$ & SA & & & LC \\
\hline Peropteryx kappleri Peters, 1867 & $\mathrm{C}$ & SA & $\operatorname{Pr}$ & & LC \\
\hline
\end{tabular}




\begin{tabular}{|c|c|c|c|c|c|}
\hline \multicolumn{6}{|l|}{ Apéndice I. Continuación... } \\
\hline & \multicolumn{2}{|c|}{ Distribución } & \multicolumn{3}{|c|}{ Estado de Conservación } \\
\hline & Ins/Cont & Continente & México & CITES & UICN \\
\hline Peropteryx macrotis (Wagner, 1843) & C & SA & & & LC \\
\hline Rhynchonycteris naso (Wied-Neuwied, 1820) & C & SA & $\operatorname{Pr}$ & & LC \\
\hline Saccopteryx bilineata (Temminck, 1838) & $\mathrm{C}$ & SA & & & LC \\
\hline Saccopteryx leptura (Schreber, 1774) & C & SA & $\operatorname{Pr}$ & & LC \\
\hline \multicolumn{6}{|l|}{ FAMILIA PHYLLOSTOMIDAE } \\
\hline \multicolumn{6}{|l|}{ SUBFAMILIA MACROTINAE } \\
\hline Macrotus californicus Baird, 1858 & C & NA & & & LC \\
\hline Macrotus waterhousii Gray, 1843 & IC & MA & & & LC \\
\hline \multicolumn{6}{|l|}{ SUBFAMILIA MICRONYCTERINAE } \\
\hline Glyphonycteris sylvestris Thomas, 1896 & C & SA & & & LC \\
\hline Lampronycteris brachyotis (Dobson, 1879) & C & SA & A & & LC \\
\hline Micronycteris microtis Miller, 1898 & IC & SA & & & LC \\
\hline Micronycteris schmidtorum Sanborn, 1935 & C & SA & A & & LC \\
\hline Trinycteris nicefori Sanborn 1949 & $\mathrm{C}$ & SA & & & LC \\
\hline \multicolumn{6}{|l|}{ SUBFAMILIA DESMODONTINAE } \\
\hline Desmodus rotundus (È. Geoffroy St.-Hilaire, 1810) & C & SA & & & LC \\
\hline Diaemus youngi (Jentink, 1893) & $\mathrm{C}$ & SA & $\operatorname{Pr}$ & & LC \\
\hline Diphylla ecaudata Spix, 1823 & C & AM & & & LC \\
\hline \multicolumn{6}{|l|}{ SUBFAMILIA VAMPYRINAE } \\
\hline Chrotopterus auritus (Peters, 1856) & C & SA & A & & LC \\
\hline Trachops cirrhosus (Spix, 1823) & C & SA & A & & LC \\
\hline Vampyrum spectrum (Linnaeus, 1758) & C & SA & $\mathrm{P}$ & & NT \\
\hline \multicolumn{6}{|l|}{ SUBFAMILIA PHYLLOSTOMINAE } \\
\hline \multicolumn{6}{|l|}{ TRIBU PHYLLOSTOMINI } \\
\hline Lonchorhina aurita Tomes, 1863 & C & SA & A & & LC \\
\hline Lophostoma brasiliense Peters, 1866 & $\mathrm{C}$ & SA & A & & LC \\
\hline Lophostoma evotis (Davis \& Carter, 1978) & C & MA & A & & LC \\
\hline Macrophyllum macrophyllum (Schinz, 1821) & C & SA & A & & LC \\
\hline Mimon cozumelae Goldman, 1914 & C & SA & A & & LC \\
\hline Mimon crenulatum (È. Geoffroy St.-Hilaire, 1810) & C & SA & A & & LC \\
\hline Phylloderma stenops Peters, 1865 & C & SA & A & & LC \\
\hline Phyllostomus discolor Wagner, 1843 & C & SA & & & LC \\
\hline Tonatia saurophila Koopman \& Williams, 1951 & IC & SA & A & & LC \\
\hline \multicolumn{6}{|l|}{ TRIBU GLOSSOPHAGINI } \\
\hline Anoura geoffroyi Gray, 1838 & $\mathrm{C}$ & SA & & & LC \\
\hline Choeroniscus godmani (Thomas, 1903) & C & SA & & & LC \\
\hline Choeronycteris mexicana Tschudi, 1844 & $\mathrm{C}$ & NA & A & & NT \\
\hline Glossophaga commissarisi Gardner, 1962 & C & SA & & & LC \\
\hline Glossophaga leachii (Gray, 1844) & C & MA & & & LC \\
\hline Glossophaga morenoi Martínez \& Villa, 1938 & C & $M X$ & & & LC \\
\hline Glossophaga soricina (Pallas, 1766) & C & SA & & & LC \\
\hline Hylonycteris underwoodi Thomas, 1903 & $\mathrm{C}$ & MA & & & LC \\
\hline
\end{tabular}




\begin{tabular}{|c|c|c|c|c|c|}
\hline \multicolumn{6}{|l|}{ Apéndice I. Continuación... } \\
\hline & \multicolumn{2}{|c|}{ Distribución } & \multicolumn{3}{|c|}{ Estado de Conservación } \\
\hline & Ins/Cont & Continente & México & CITES & UICN \\
\hline Leptonycteris nivalis (Saussure, 1860) & C & NA & A & & EN \\
\hline Leptonycteris yerbabuenae Martínez \& Villa, 1940 & IC & AM & A & & VU \\
\hline Lichonycteris obscura Thomas, 1895 & C & SA & & & LC \\
\hline Musonycteris harrisoni Schaldach \& McLaughlin, 1960 & C & $M X$ & $\mathrm{P}$ & & $\mathrm{VU}$ \\
\hline \multicolumn{6}{|l|}{ TRIBU STENODERMATINI } \\
\hline Artibeus hirsutus Andersen, 1906 & $\mathrm{C}$ & $M X$ & & & LC \\
\hline Artibeus jamaicensis Leach, 1821 & IC & SA & & & LC \\
\hline Artibeus lituratus (Olfers, 1818) & IC & SA & & & LC \\
\hline Carollia perspicillata (Linnaeus, 1758) & C & SA & & & LC \\
\hline Carollia sowelli Baker et al., 2002 & C & MA & & & LC \\
\hline Carollia subrufa (Hahn, 1905) & $\mathrm{C}$ & MA & & & LC \\
\hline Centurio senex Gray, 1842 & C & SA & & & LC \\
\hline Chiroderma salvini Dobson, 1878 & C & SA & & & LC \\
\hline Chiroderma villosum Peters, 1860 & $\mathrm{C}$ & SA & & & LC \\
\hline Dermanura azteca (Andersen, 1906) & C & MA & & & LC \\
\hline Dermanura phaeotis (Miller, 1902) & IC & SA & & & LC \\
\hline Dermanura tolteca (Saussure, 1860) & $\mathrm{C}$ & MA & & & LC \\
\hline Dermanura watsoni (Thomas, 1901) & C & SA & $\operatorname{Pr}$ & & LC \\
\hline Enchisthenes hartii (Thomas, 1892) & C & SA & $\operatorname{Pr}$ & & LC \\
\hline Platyrrhinus helleri (Peters, 1866) & $\mathrm{C}$ & SA & & & LC \\
\hline Sturnira hondurensis Goodwin, 1940 & C & MA & & & LC \\
\hline Sturnira lilium (È. Geoffroy St.-Hilaire, 1810) & $\mathrm{C}$ & SA & & & LC \\
\hline Uroderma bilobatum Peters, 1866 & $\mathrm{C}$ & SA & & & LC \\
\hline Uroderma magnirostrum Davis, 1968 & C & SA & & & LC \\
\hline Vampyressa thyone Thomas, 1909 & C & SA & & & LC \\
\hline Vampyrodes major (Thomas, 1889) & $\mathrm{C}$ & SA & & & LC \\
\hline \multicolumn{6}{|l|}{ FAMILIA MORMOOPIDAE } \\
\hline Mormoops megalophylla (Peters, 1864) & IC & AM & & & LC \\
\hline Pteronotus davyi Gray, 1838 & IC & SA & & & LC \\
\hline Pteronotus gymnonotus (Wagner, 1843) & C & SA & A & & LC \\
\hline Pteronotus parnellii (Gray, 1843) & IC & SA & & & LC \\
\hline Pteronotus personatus (Wagner, 1843) & IC & SA & & & LC \\
\hline \multicolumn{6}{|l|}{ FAMILIA NOCTILIONIDAE } \\
\hline Noctilio albiventris Desmarest, 1818 & $\mathrm{C}$ & SA & $\operatorname{Pr}$ & & LC \\
\hline Noctilio leporinus (Linnaeus, 1758) & C & SA & & & LC \\
\hline \multicolumn{6}{|l|}{ FAMILIA THYROPTERIDAE } \\
\hline Thyroptera tricolor Spix, 1823 & $\mathrm{C}$ & SA & $\operatorname{Pr}$ & & LC \\
\hline \multicolumn{6}{|l|}{ FAMILIA NATALIDAE } \\
\hline Natalus mexicanus Miller, 1902 & C & MA & & & LC \\
\hline \multicolumn{6}{|l|}{ FAMILIA MOLOSSIDAE } \\
\hline \multicolumn{6}{|l|}{ SUBFAMILIA MOLOSSINAE } \\
\hline Cynomops mexicanus (Jones \& Genoways, 1967) & $\mathrm{C}$ & $M X$ & $\operatorname{Pr}$ & & LC \\
\hline
\end{tabular}




\begin{tabular}{|c|c|c|c|c|c|}
\hline \multicolumn{6}{|l|}{ Apéndice I. Continuación... } \\
\hline & \multicolumn{2}{|c|}{ Distribución } & \multicolumn{3}{|c|}{ Estado de Conservación } \\
\hline & Ins/Cont & Continente & México & CITES & UICN \\
\hline Eumops auripendulus (Shaw, 1800) & C & SA & & & LC \\
\hline Eumops ferox (Gundlach, 1862) & $\mathrm{C}$ & AM & & & \\
\hline Eumops hansae Sanborn, 1932 & $\mathrm{C}$ & SA & & & LC \\
\hline Eumops nanus (Miller, 1900) & IC & SA & $\operatorname{Pr}$ & & \\
\hline Eumops perotis (Schinz, 1821) & $\mathrm{C}$ & AM & & & LC \\
\hline Eumops underwoodi Goodwin, 1940 & $\mathrm{C}$ & NA & & & LC \\
\hline Molossus alvarezi González-Ruiz et al., 2011 & $\mathrm{C}$ & MA & & & \\
\hline Molossus aztecus Saussure, 1860 & IC & MA & & & LC \\
\hline Molossus coibensis J. A. Allen, 1904 & $\mathrm{C}$ & SA & & & LC \\
\hline Molossus molossus (Pallas, 1766) & $\mathrm{C}$ & SA & & & LC \\
\hline Molossus rufus E. Geoffroy, 1805 & $\mathrm{C}$ & SA & & & LC \\
\hline Molossus sinaloae J. A. Allen, 1906 & $\mathrm{C}$ & SA & & & LC \\
\hline Nyctinomops aurispinosus (Peale, 1848) & $\mathrm{C}$ & SA & & & LC \\
\hline Nyctinomops femorosaccus (Merriam, 1889) & $\mathrm{C}$ & NA & & & LC \\
\hline Nyctinomops laticaudatus (È. Geoffroy St.-Hilaire, 1805) & C & SA & & & LC \\
\hline Nyctinomops macrotis (Gray, 1840) & C & AM & & & LC \\
\hline Promops centralis Thomas, 1915 & $\mathrm{C}$ & SA & & & LC \\
\hline \multicolumn{6}{|l|}{ SUBFAMILIA TADARINAE } \\
\hline Tadarida brasiliensis (I. Geoffroy St.-Hilaire, 1824) & C & NA & & & LC \\
\hline \multicolumn{6}{|l|}{ FAMILIA VESPERTILIONIDAE } \\
\hline \multicolumn{6}{|l|}{ SUBFAMILIA MYOTINAE } \\
\hline Myotis albescens (È. Geoffroy St.-Hilaire, 1806) & $\mathrm{C}$ & SA & $\operatorname{Pr}$ & & LC \\
\hline Myotis auriculus Baker \& Stains, 1955 & $\mathrm{C}$ & NA & & & LC \\
\hline Myotis californicus (Audubon \& Bachman, 1842) & $\mathrm{C}$ & $\mathrm{NA}$ & & & LC \\
\hline Myotis carteri La Val, 1973 & $\mathrm{C}$ & $M X$ & $\operatorname{Pr}$ & & \\
\hline Myotis elegans Hall, 1962 & $\mathrm{C}$ & MA & & & LC \\
\hline Myotis evotis (H. Allen, 1864) & $\mathrm{C}$ & NA & $\operatorname{Pr}$ & & LC \\
\hline Myotis findleyi Bogan, 1978 & 1 & $M X$ & & & EN \\
\hline Myotis fortidens Miller \& Allen, 1928 & $\mathrm{C}$ & MA & & & LC \\
\hline Myotis keaysi J. A. Allen, 1914 & $\mathrm{C}$ & SA & & & LC \\
\hline Myotis melanorhinus (Merriam, 1890) & $\mathrm{C}$ & NA & & & LC \\
\hline Myotis nigricans (Schinz, 1821) & $\mathrm{C}$ & SA & & & LC \\
\hline Myotis occultus Hollister, 1909 & $\mathrm{C}$ & $\mathrm{NA}$ & & & LC \\
\hline Myotis peninsularis Miller, 1898 & $\mathrm{C}$ & $M X$ & & & EN \\
\hline Myotis planiceps Baker, 1955 & $\mathrm{C}$ & $M X$ & $\mathrm{P}$ & & EN \\
\hline Myotis thysanodes Miller, 1897 & $\mathrm{C}$ & NA & & & LC \\
\hline Myotis velifer (J. A. Allen, 1890) & $\mathrm{C}$ & NA & & & LC \\
\hline Myotis vivesi Menegaux, 1901 & $\mathrm{C}$ & $M X$ & $\mathrm{P}$ & & $\mathrm{VU}$ \\
\hline Myotis volans (H. Allen, 1866) & $\mathrm{C}$ & NA & & & LC \\
\hline Myotis yumanensis (H. Allen, 1864) & $\mathrm{C}$ & NA & & & LC \\
\hline \multicolumn{6}{|l|}{ SUBFAMILIA VESPERTILIONINAE } \\
\hline Baeodon alleni Thomas, 1892 & $\mathrm{C}$ & $M X$ & & & LC \\
\hline Baeodon gracilis Miller, 1897 & $\mathrm{C}$ & $M X$ & & & LC \\
\hline
\end{tabular}




\begin{tabular}{|c|c|c|c|c|c|}
\hline \multicolumn{6}{|l|}{ Apéndice I. Continuación... } \\
\hline & \multicolumn{2}{|c|}{ Distribución } & \multicolumn{3}{|c|}{ Estado de Conservación } \\
\hline & Ins/Cont & Continente & México & CITES & UICN \\
\hline Corynorhinus mexicanus G. M. Allen, 1916 & $\mathrm{C}$ & MX & & & NT \\
\hline Corynorhinus townsendii (Cooper, 1837) & $\mathrm{C}$ & NA & & & LC \\
\hline Eptesicus brasiliensis (Desmarest, 1819) & C & SA & & & LC \\
\hline Eptesicus furinalis (d'Orbigny \& Gervais, 1847) & $\mathrm{C}$ & SA & & & $\mathrm{LC}$ \\
\hline Eptesicus fuscus (Palisot de Beauvois, 1796) & C & AM & & & LC \\
\hline Euderma maculatum (J. A. Allen, 1891) & $\mathrm{C}$ & NA & $\operatorname{Pr}$ & & LC \\
\hline Idionycteris phyllotis (G.M. Allen, 1916) & C & $\mathrm{NA}$ & & & LC \\
\hline Lasionycteris noctivagans (Le Conte, 1831) & C & $\mathrm{NA}$ & $\operatorname{Pr}$ & & $\mathrm{LC}$ \\
\hline Lasiurus blossevillii (Lesson \& Garnot, 1826) & IC & NA & & & LC \\
\hline Lasiurus borealis (Müller, 1776) & C & $\mathrm{NA}$ & & & $\mathrm{LC}$ \\
\hline Lasiurus cinereus (Palisot de Beauvois, 1796) & $\mathrm{C}$ & AM & & & LC \\
\hline Lasiurus ega (Gervais, 1856) & $\mathrm{C}$ & AM & & & LC \\
\hline Lasiurus intermedius H. Allen, 1862 & C & NA & & & LC \\
\hline Lasiurus xanthinus (Thomas, 1897) & C & $\mathrm{NA}$ & & & LC \\
\hline Nycticeius humeralis (Rafinesque, 1818) & C & $\mathrm{NA}$ & & & $\mathrm{LC}$ \\
\hline Parastrellus hesperus (H. Allen, 1864) & C & $\mathrm{NA}$ & & & LC \\
\hline Perimyotis subflavus (F. Cuvier, 1832) & C & $\mathrm{NA}$ & & & $\mathrm{LC}$ \\
\hline Rhogeessa aeneus Goodwin, 1958 & $\mathrm{C}$ & MX & & & $\mathrm{LC}$ \\
\hline Rhogeessa bickhami Baker et al., 2012 & C & MX & & & \\
\hline Rhogeessa genowaysi Baker, 1984 & $\mathrm{C}$ & MX & A & & EN \\
\hline Rhogeessa mira La Val, 1973 & C & MX & $\operatorname{Pr}$ & & $\mathrm{VU}$ \\
\hline Rhogeessa parvula H. Allen, 1866 & IC & MX & & & $\mathrm{LC}$ \\
\hline Rhogeessa tumida H. Allen, 1866 & $\mathrm{C}$ & MA & & & LC \\
\hline \multicolumn{6}{|l|}{ FAMILIA ANTROZOIDAE } \\
\hline Antrozous pallidus (Le Conte, 1856) & IC & NA & & & LC \\
\hline Bauerus dubiaquercus (Van Gelder, 1959) & IC & MA & & & NT \\
\hline
\end{tabular}




\section{APÉNDICE II}

\section{Mapas de distribución de los mamíferos de México}

ORDEN DIDELPHIMORPHIA

FAMILIA DIDELPHIDAE

Chironectes minimus

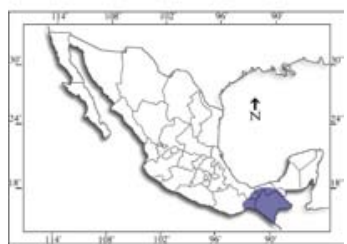

Metachirus nudicaudatus

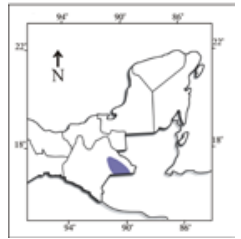

ORDEN SIRENIA

FAMILIA TRICHECHIDAE

Trichechus manatus

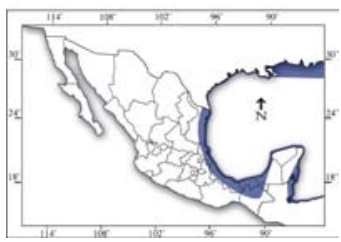

ORDEN PILOSA

FAMILIA MYRMECOPHAGIDAE
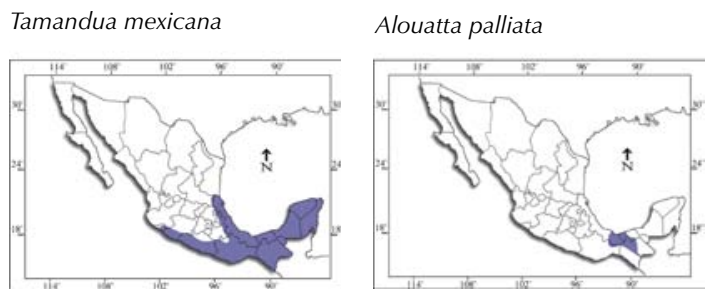

Didelphis virginiana

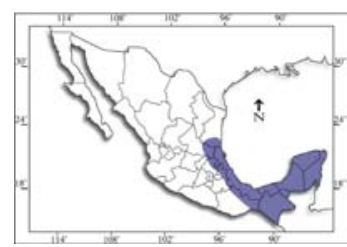

Philander opossum

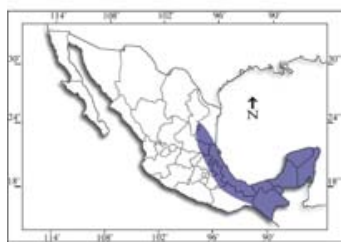

ORDEN CINGULATA

FAMILIA DASYPODIDAE

Dasypus novemcinctus

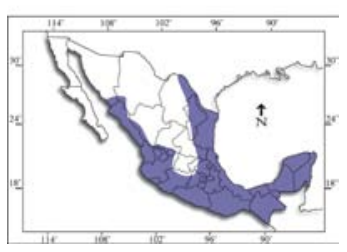

ORDEN LAGOMORPHA

FAMILIA LEPORIDAE

Lepus alleni

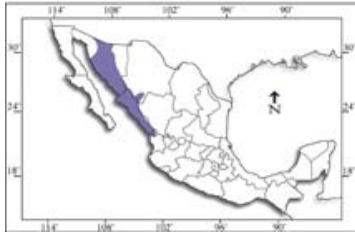

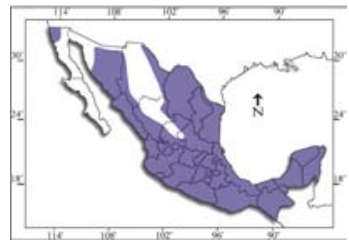

Tlacuatzin canescens
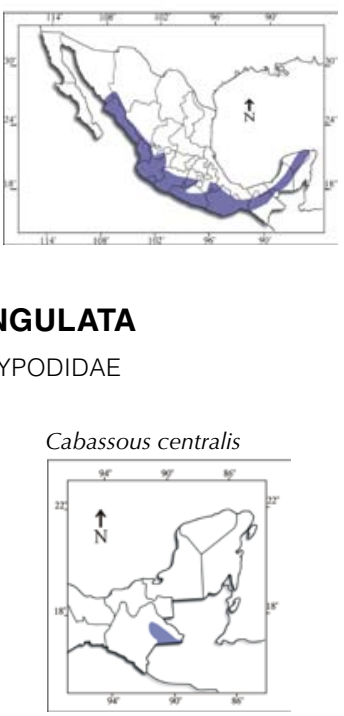

ORDEN PRIMATES

FAMILIA ATELIDAE

Alouatta pigra
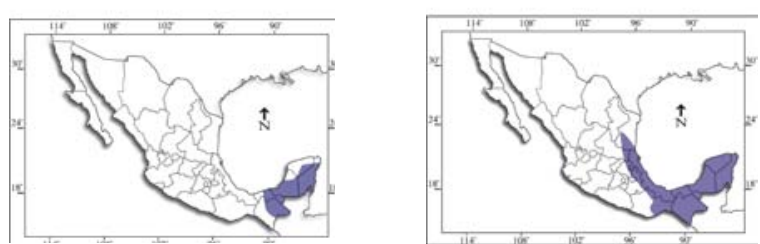

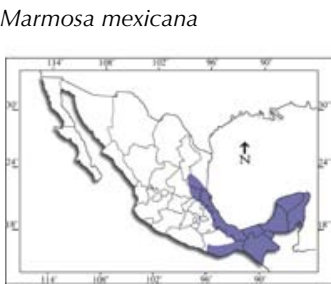

Caluromys derbianus

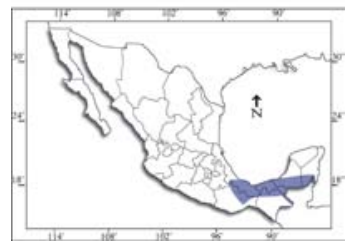

ORDEN PILOSA

FAMILIA CYCLOPEDIDAE

Cyclopes didactylus

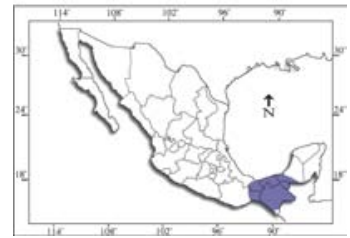




\section{ORDEN LAGOMORPHA}

FAMILIA LEPORIDAE
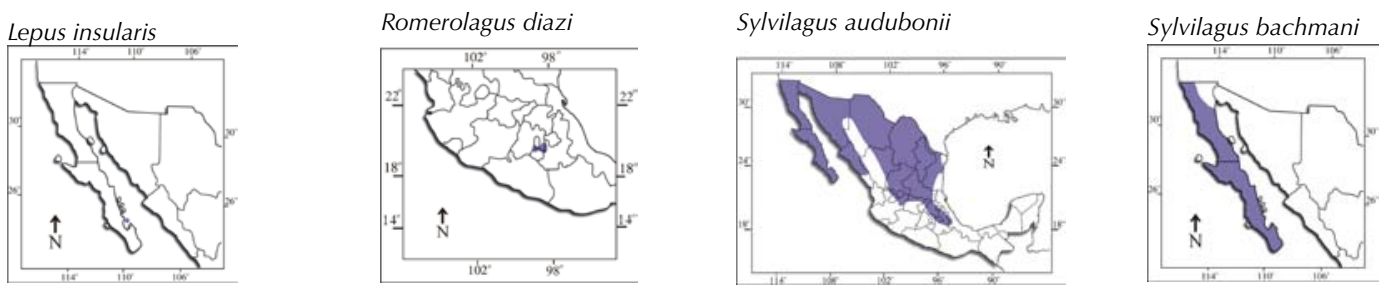

Sylvilagus cunicularius
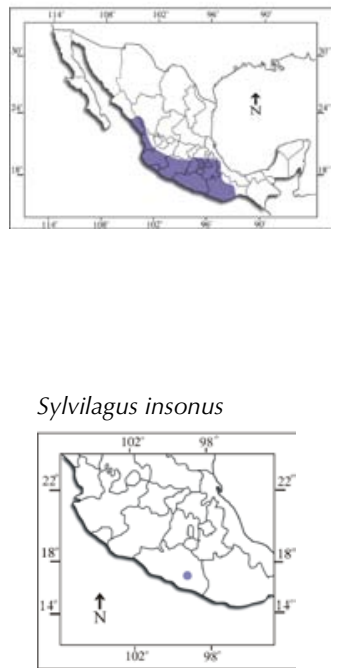

Ammospermophilus insularis


Sylvilagus floridanus
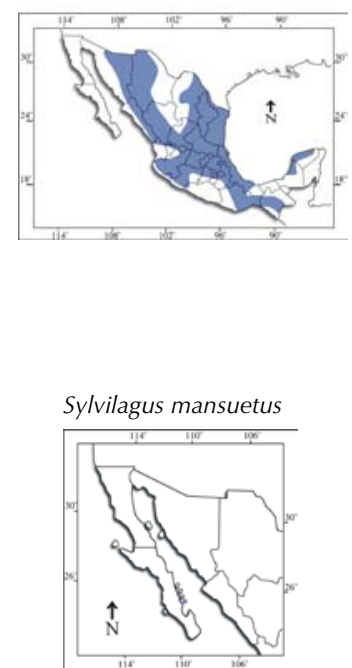

Sylvilagus gabii

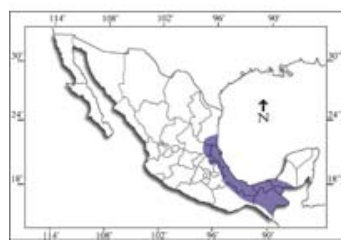

Sylvilagus robustus

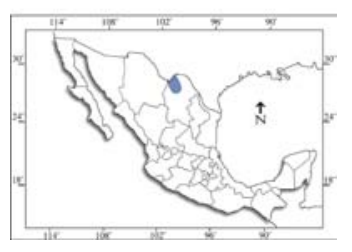

Ammospermophilus leucurus

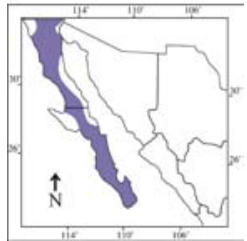

Callospermophilus madrensis
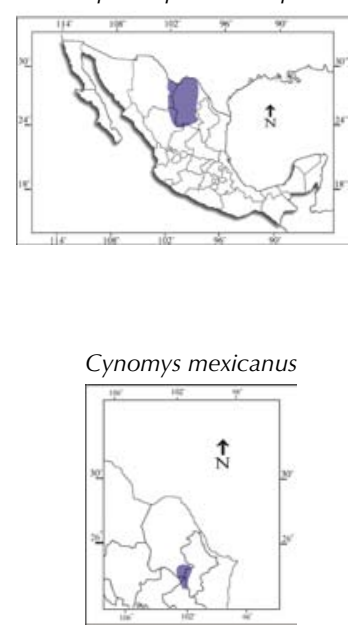

Sylvilagus graysoni

ORDEN RODENTIA

FAMILIA SCIURIDAE

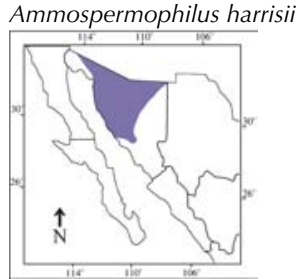

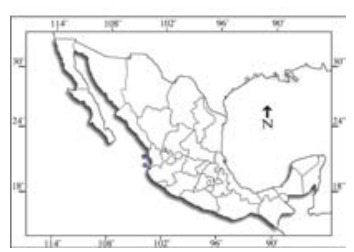




\section{ORDEN RODENTIA}

FAMILIA SCIURIDAE

Ictidomys parvidens

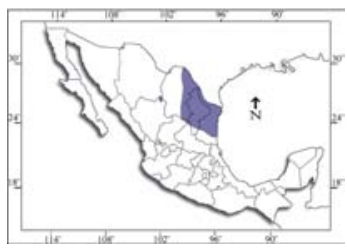

Neotamias merriami

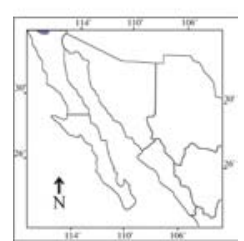

Otospermophilus atricapillus

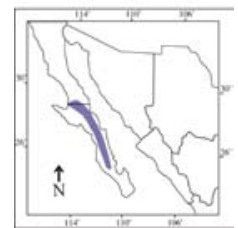

Neotamias bulleri

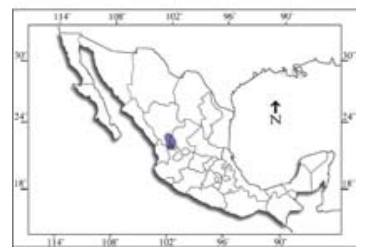

Neotamias obscurus

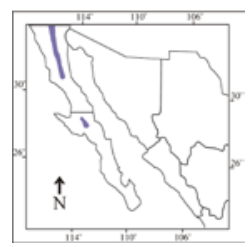

Neotamias dorsalis

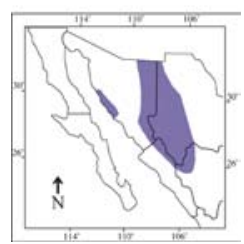

Notocitellus adocetus

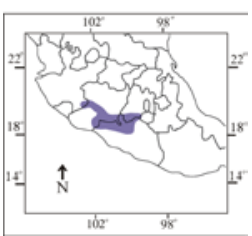

Neotamias durangae

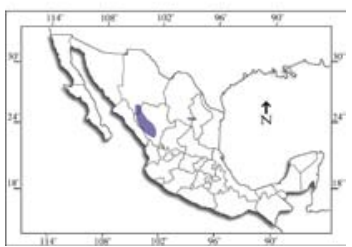

Notocitellus annulatus

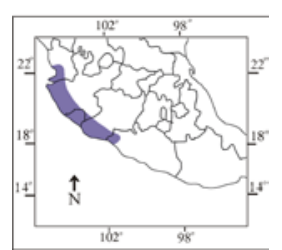

Otospermophilus beecheyi

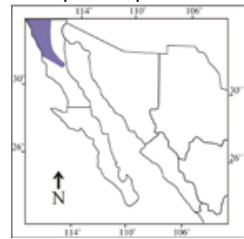

Otospermophilus variegatus

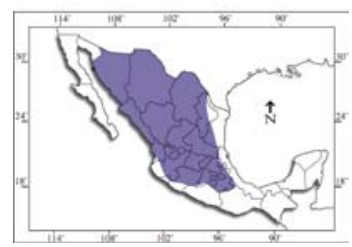

Sciurus aureogaster

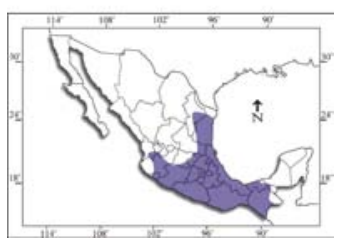

Sciurus nayaritensis

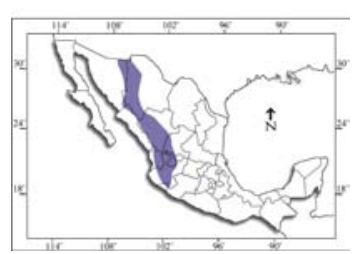

Sciurus aberti

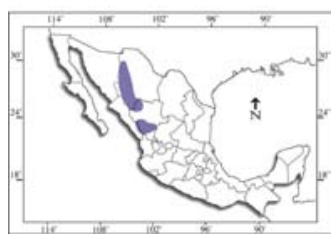

Sciurus alleni

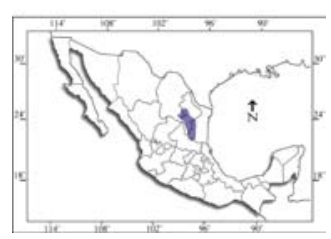

Sciurus deppei

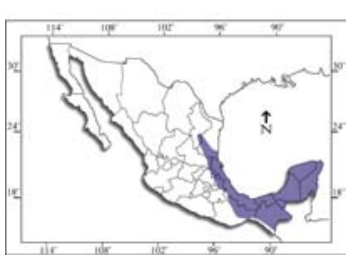

Sciurus arizonensis

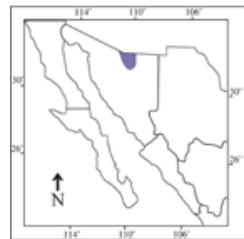

Sciurus griseus

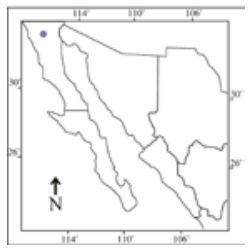

Sciurus colliaei

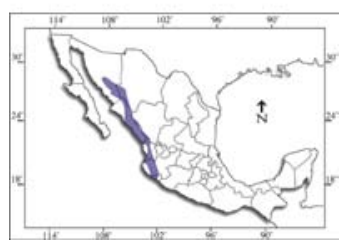

Sciurus niger

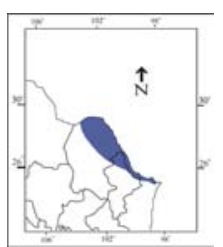


Sciurus oculatus

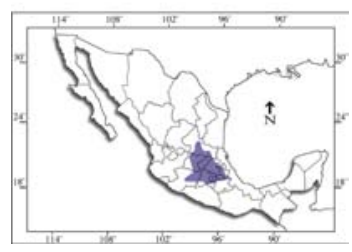

Sciurus variegatoides

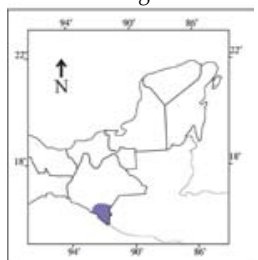

Sciurus yucatanensis

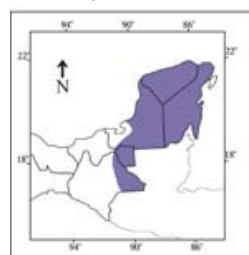

Tamiasciurus mearnsi

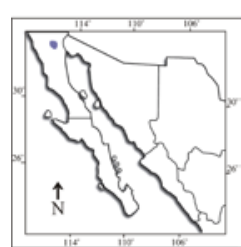

ORDEN RODENTIA

FAMILIA CASTORIDAE

Xerospermophilus tereticaudus Castor canadensis
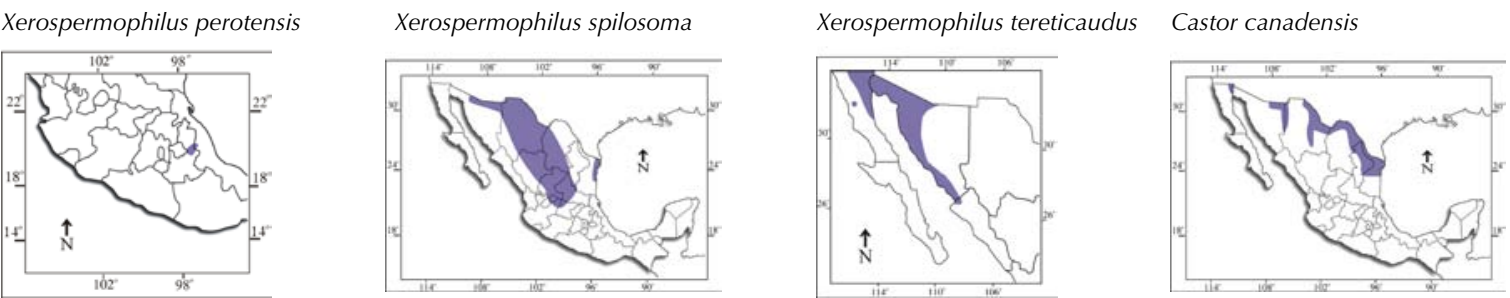

\section{ORDEN RODENTIA}

FAMILIA HETEROMYIDAE

Dipodomys compactus

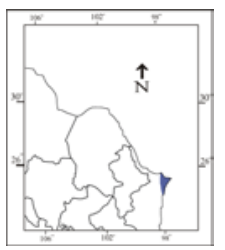

Dipodomys merriami

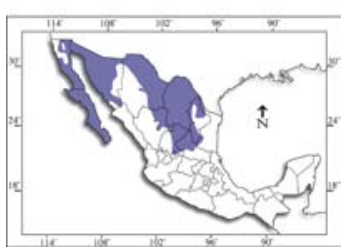

Dipodomys simulans

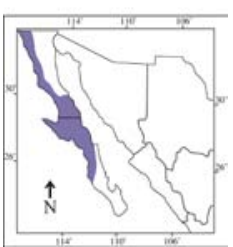

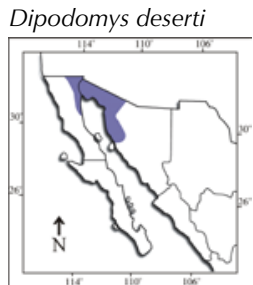

Dipodomys gravipes

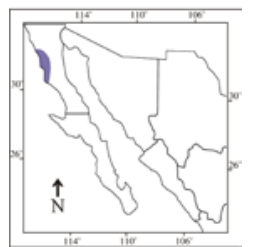

Dipodomys insularis

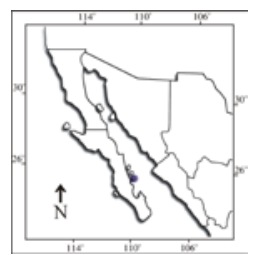

Dipodomys nelsoni

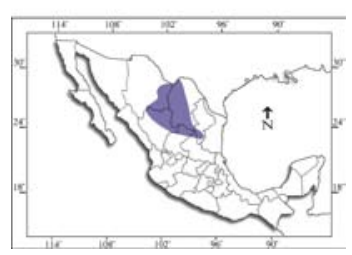

Dipodomys ordii

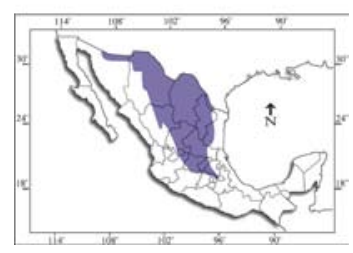

Dipodomys phillipsii

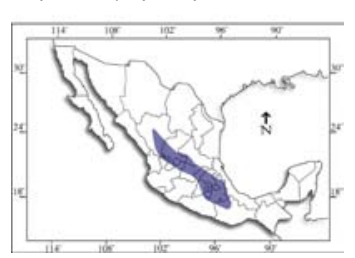


Heteromys nelsoni

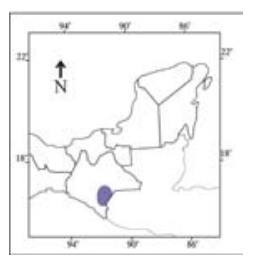

Liomys spectabilis

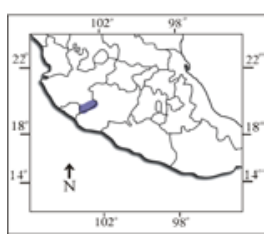

Liomys irroratus

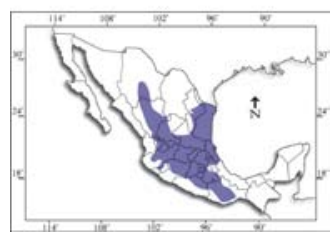

Liomys pictus

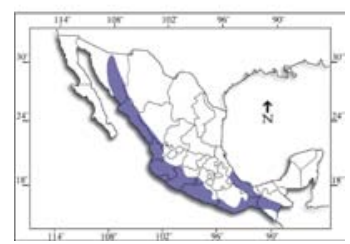

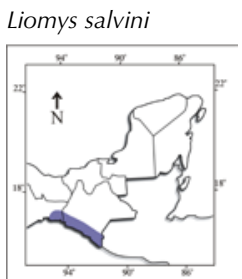

Chaetodipus anthonyi

Chaetodipus arenarius

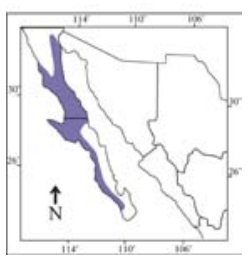

Chaetodipus artus

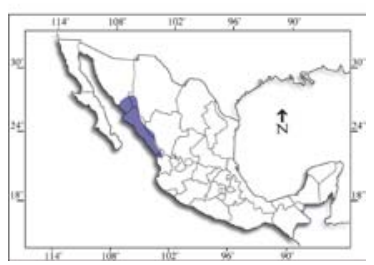

Chaetodipus baileyi

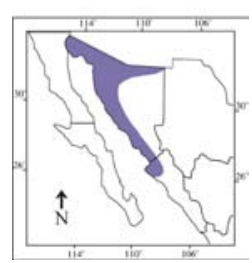

Chaetodipus fallax

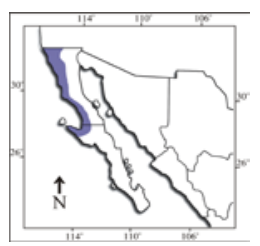

Chaetodipus californicus

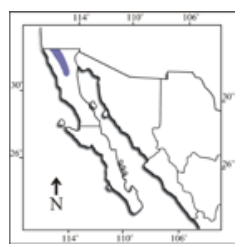

Chaetodipus dalquesti

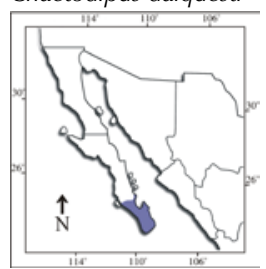

Chaetodipus eremicus

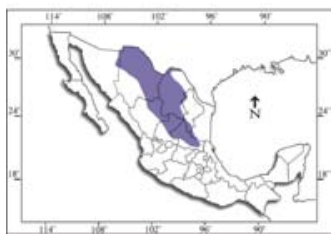

Chaetodipus formosus

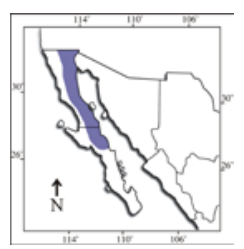

Chaetodipus goldmani

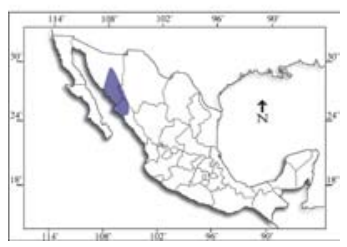

Chaetodipus hispidus

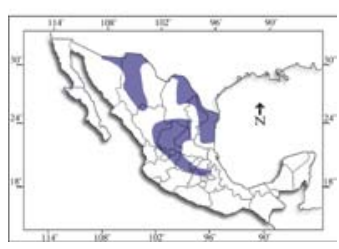

Chaetodipus intermedius

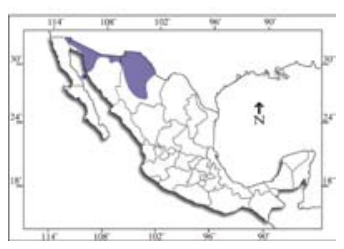

Chaetodipus lineatus

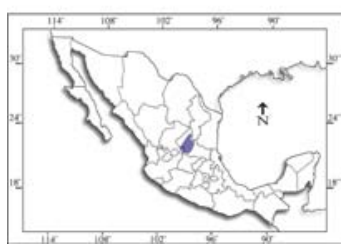

Chaetodipus nelsoni

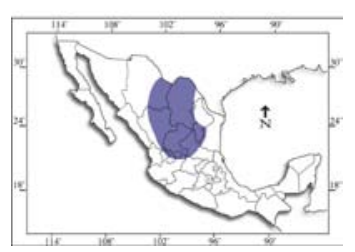

Chaetodipus penicillatus

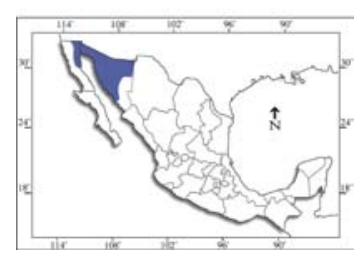


Chaetodipus pernix

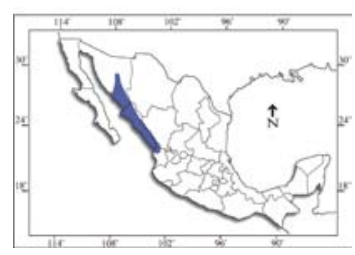

Perognathus flavescens

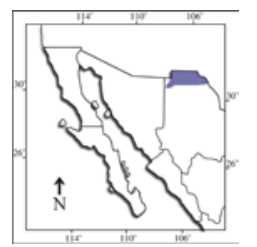

ORDEN RODENTIA

FAMILIA GEOMYIDAE

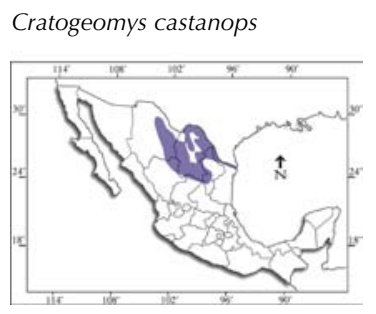

Chaetodipus rudinoris

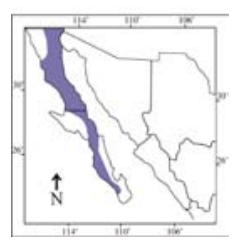

Perognathus flavus

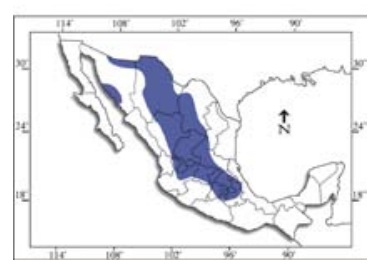

Chaetodipus spinatus

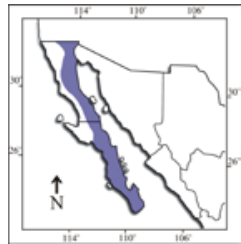

Perognathus longimembris

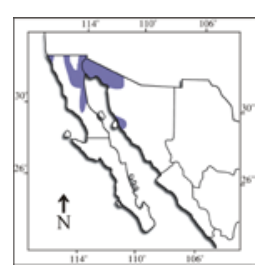

Perognathus amplus

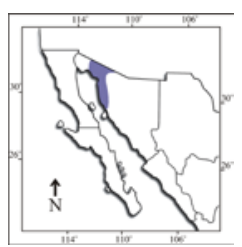

Perognathus merriami

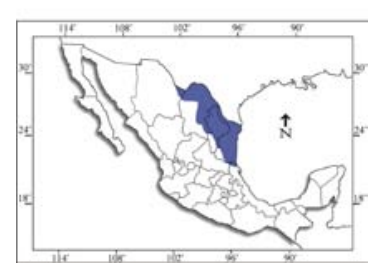

Cratogeomys fumosus

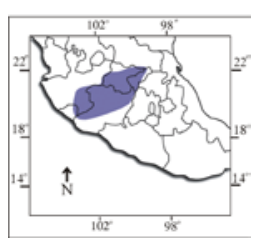

Cratogeomys goldmani

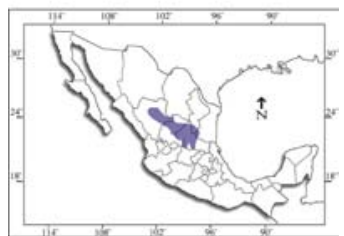

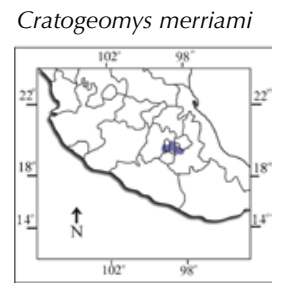

Cratogeomys perotensis

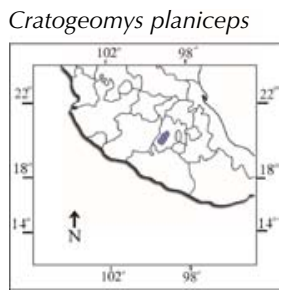

Geomys arenarius

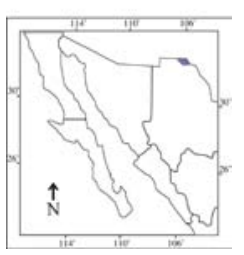

Orthogeomys cuniculus

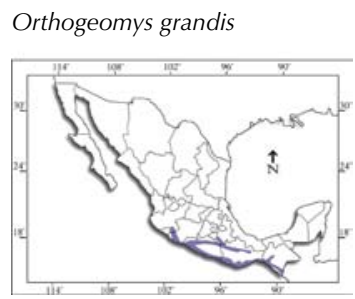


Orthogeomys hispidus

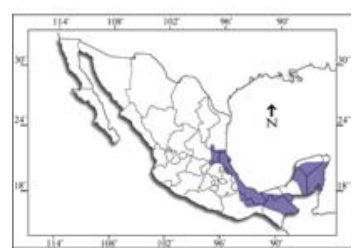

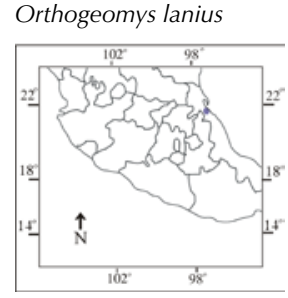

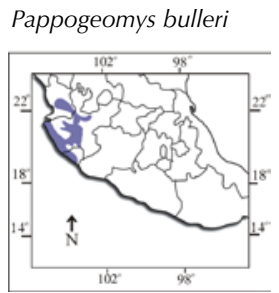

Thomomys atrovarius

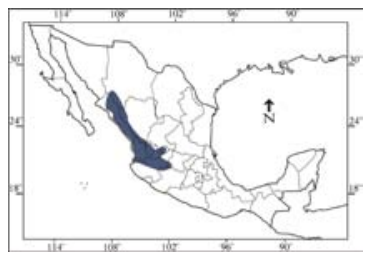

Thomomys bottae

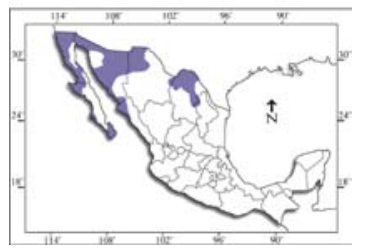

Thomomys sheldoni

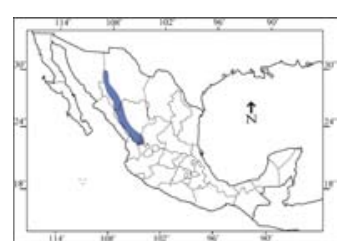

Thomomys umbrinus

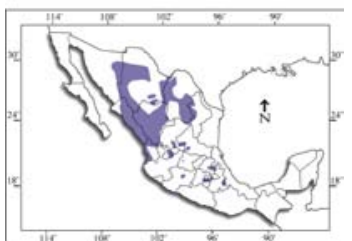

Zygogeomys trichopus

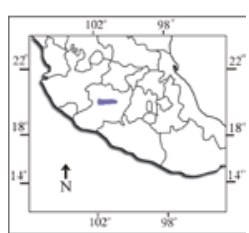

ORDEN RODENTIA

FAMILIA MURIDAE

Microtus californicus

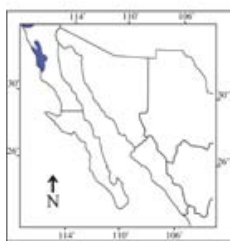

Microtus guatemalensis

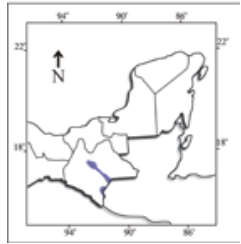

Microtus mexicanus

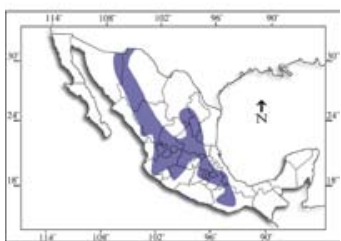

Microtus oaxacensis

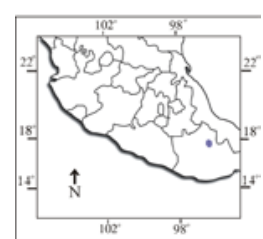

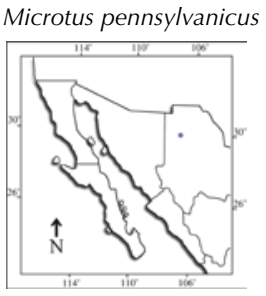

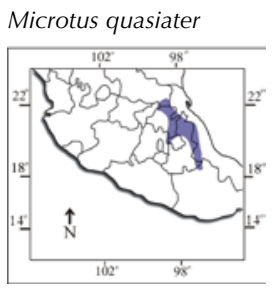

Microtus umbrosus

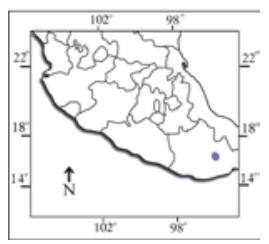

Ondatra zibethicus

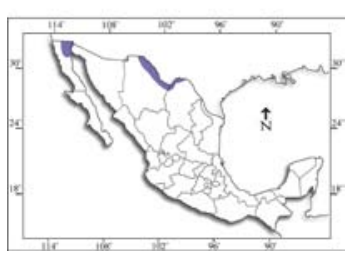

Baiomys musculus

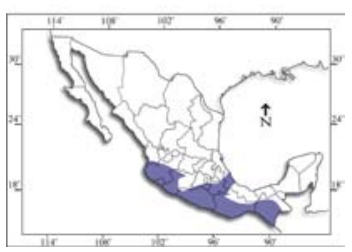

Baiomys taylori

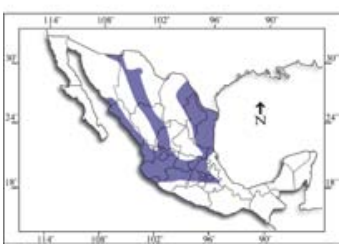

Habromys chinanteco

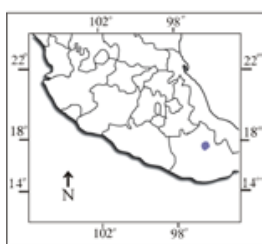

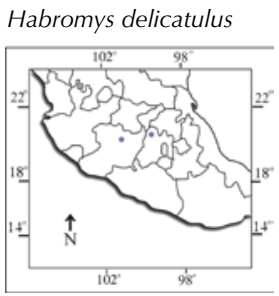



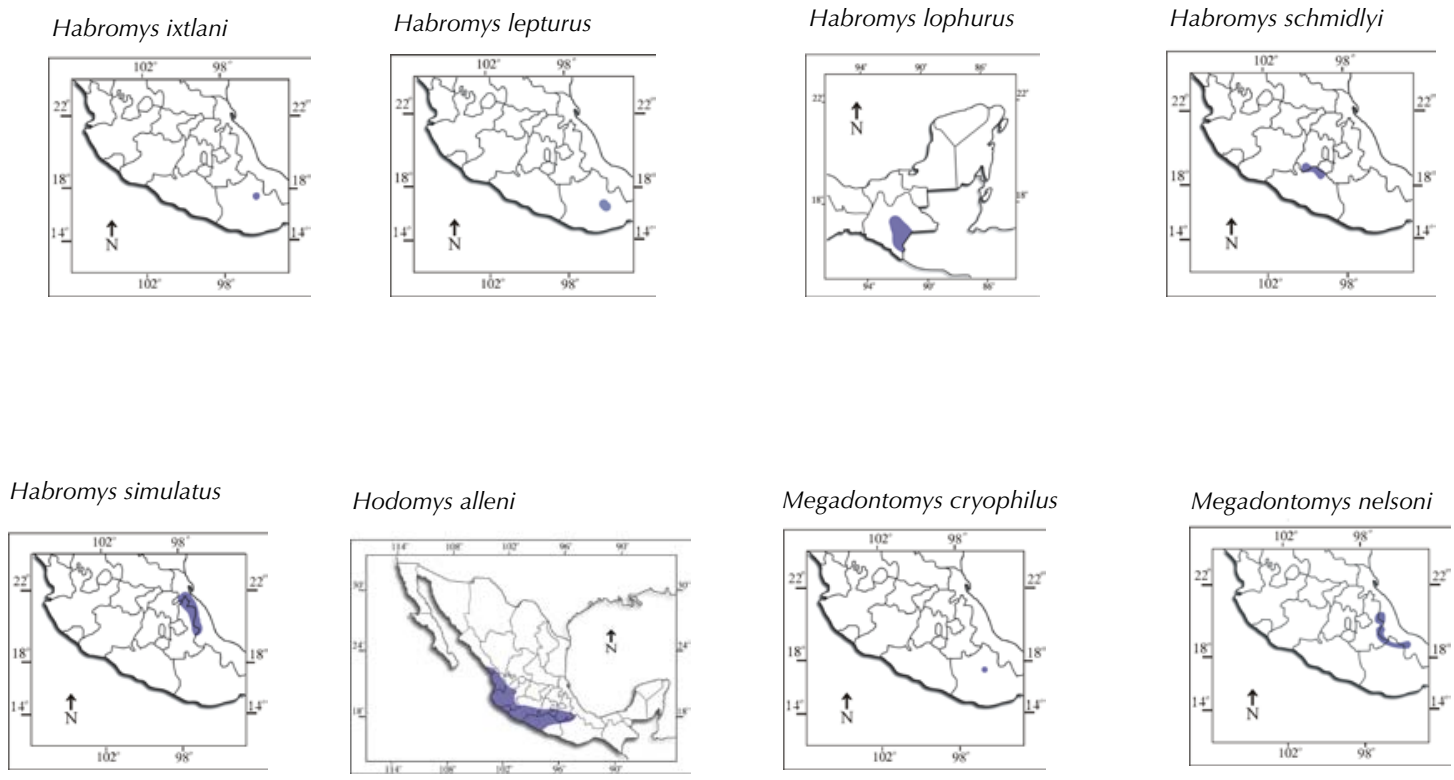

Megadontomys thomasi
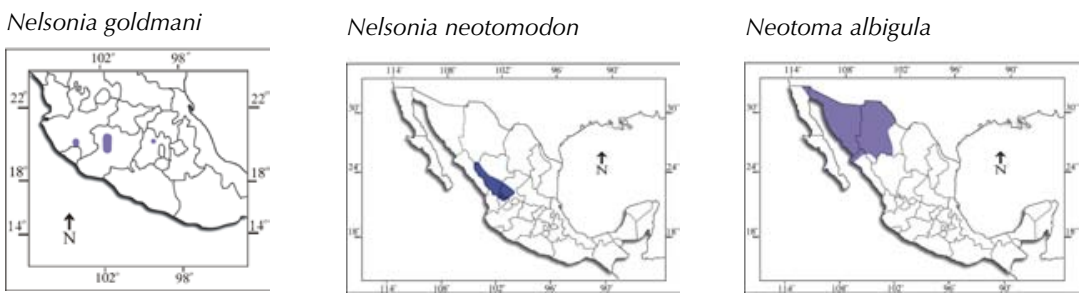

Neotoma angustapalata

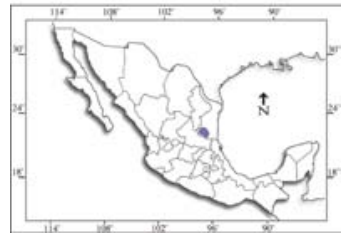

Neotoma bryanti

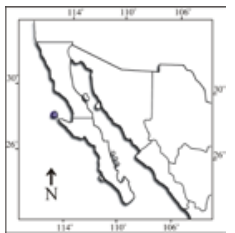

Neotoma devia

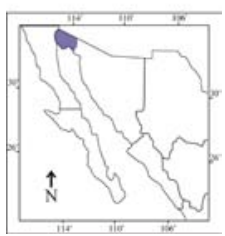

Neotoma goldmani

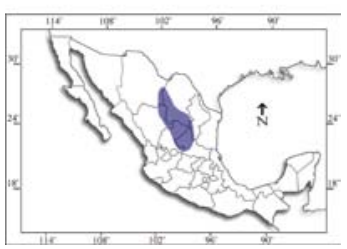

Neotoma insularis

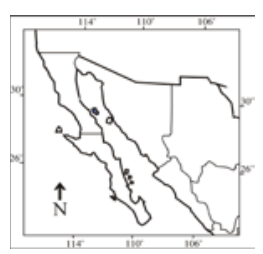

Neotoma isthmica

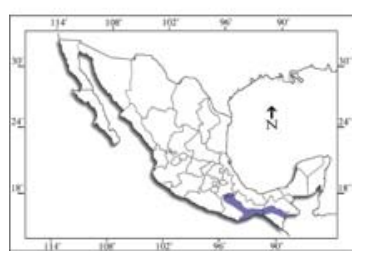

Neotoma lepida

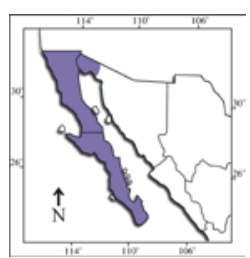

Neotoma leucodon

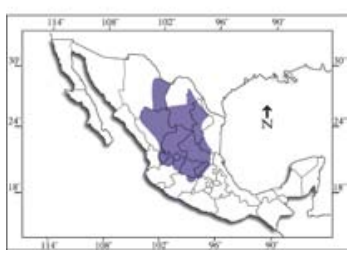



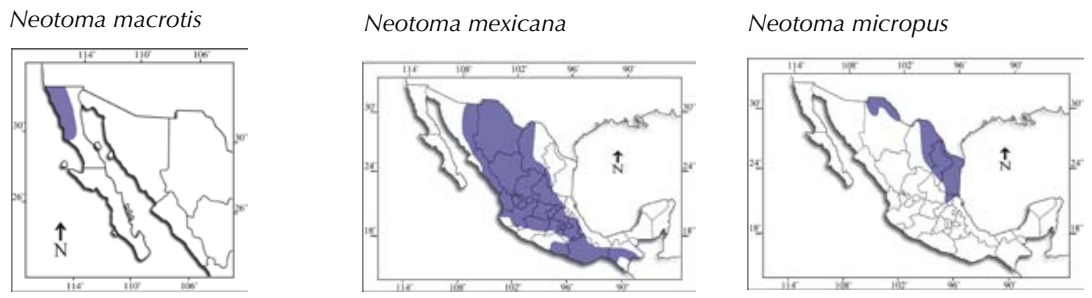

Neotoma nelsoni

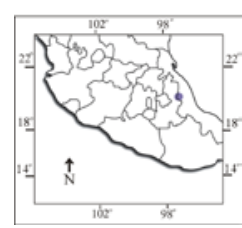

Neotoma palatina

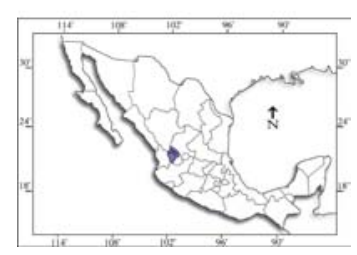

Neotoma phenax

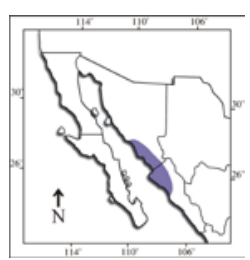

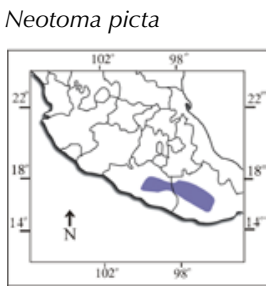

Neotomodon alstoni

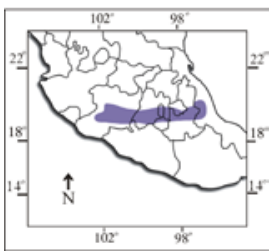

Onychomys arenicola

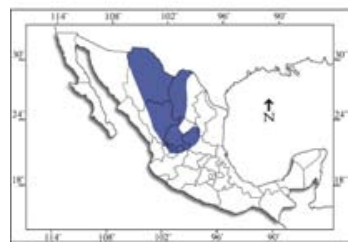

Onychomys leucogaster

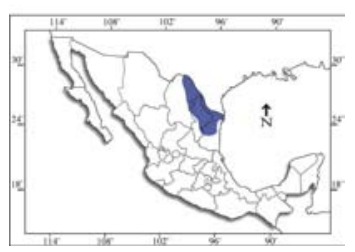

Oligoryzomys fulvescens

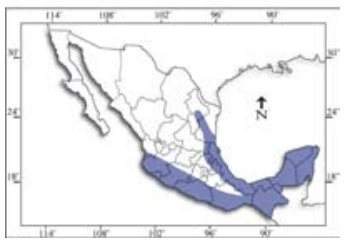

Onychomys torridus

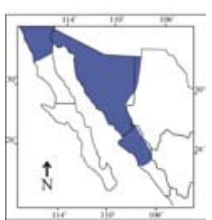

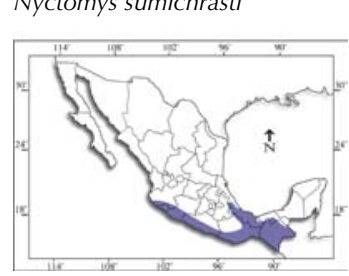

Oryzomys couesi

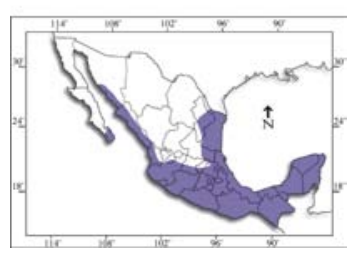

Oryzomys albiventer

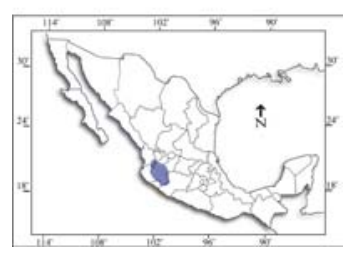

Oryzomys alfaroi

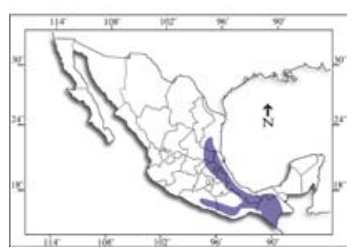

Oryzomys chapmani

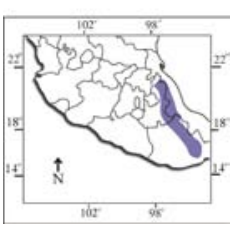

Oryzomys melanotis

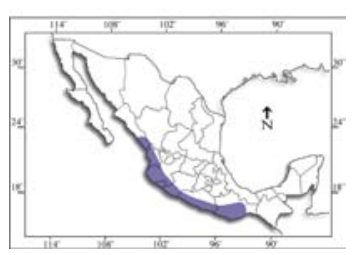

Oryzomys mexicanus

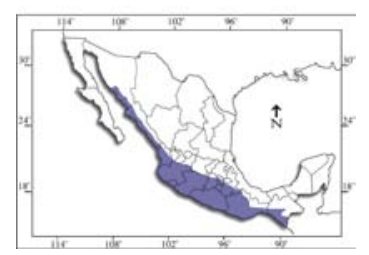

Oryzomys nelsoni

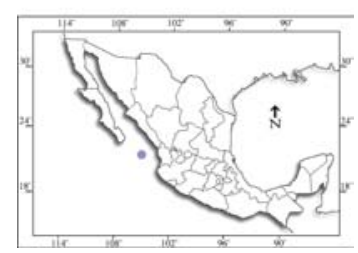



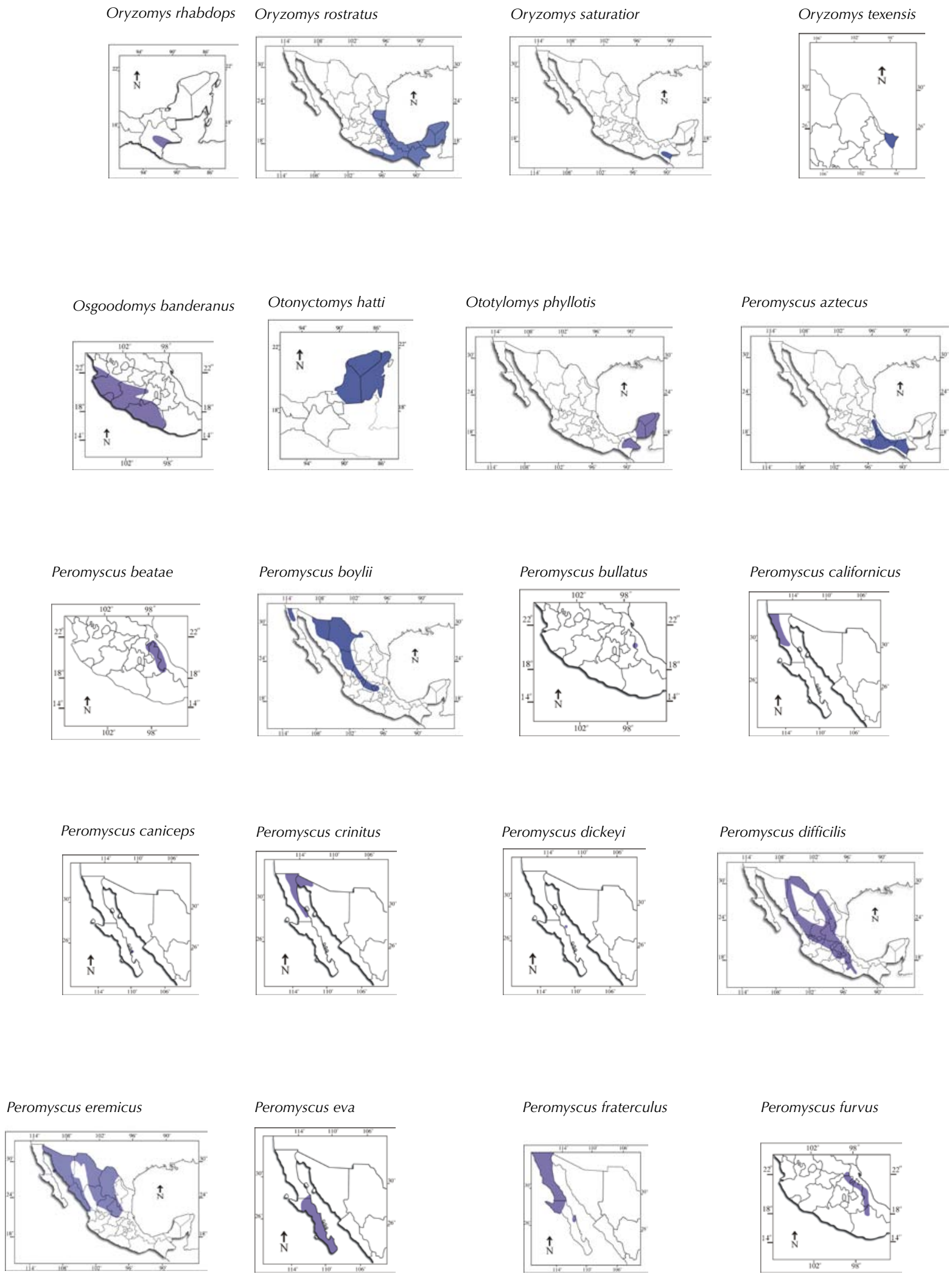

Peromyscus fraterculus

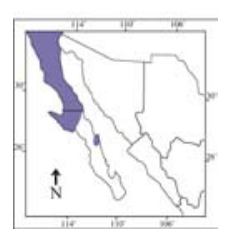

Peromyscus furvus

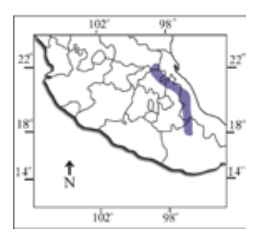


Peromyscus gratus
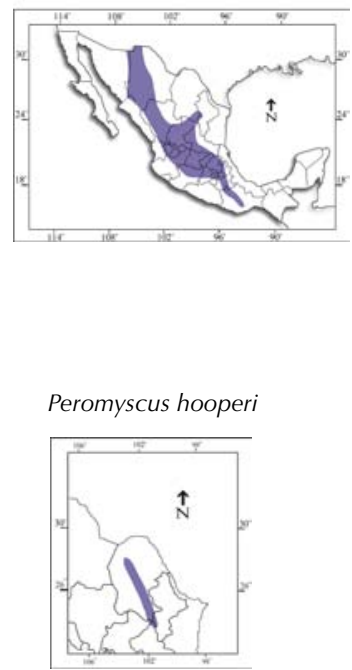

Peromyscus guardia

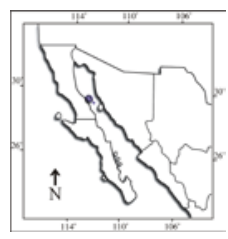

Peromyscus guatemalensis



Peromyscus gymnotis

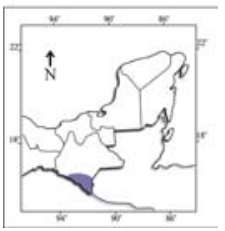

Peromyscus interparietalis

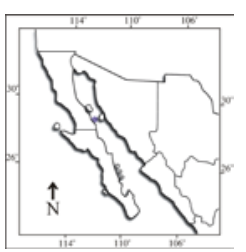

Peromyscus leucopus

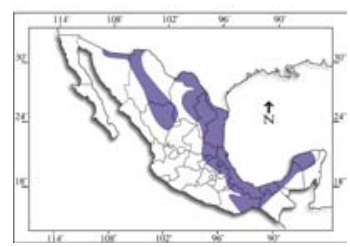

Peromyscus levipes

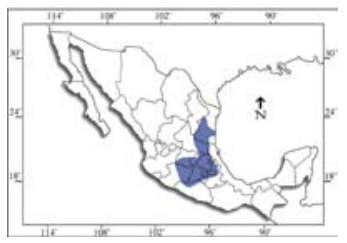

Peromyscus hylocetes

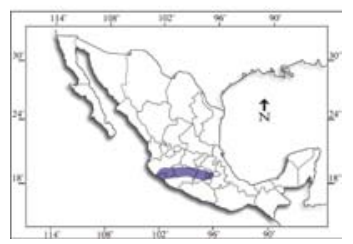

Peromyscus madrensis

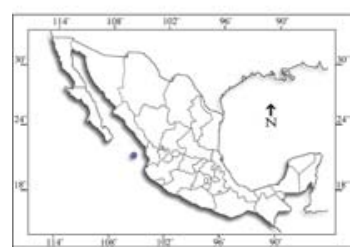

Peromyscus maniculatus

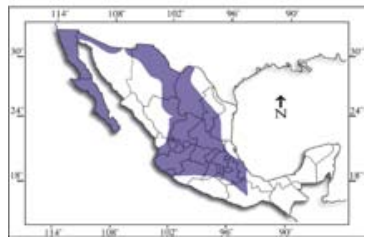

Peromyscus megalops

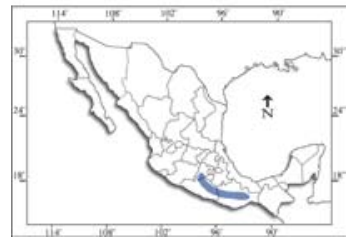

Peromyscus mekisturus

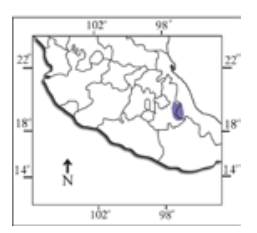

Peromyscus melanocarpus

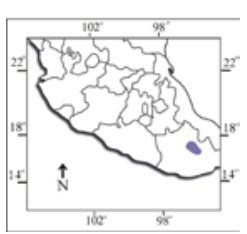

Peromyscus melanophrys

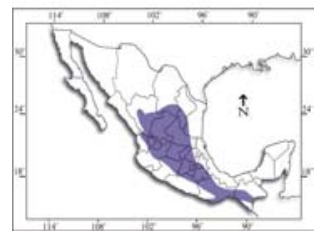

Peromyscus melanotis

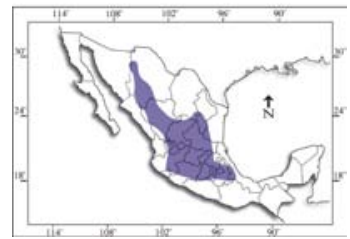

Peromyscus melanurus

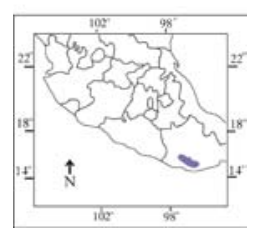

Peromyscus merriami

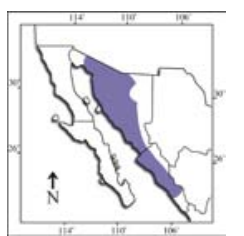

Peromyscus mexicanus

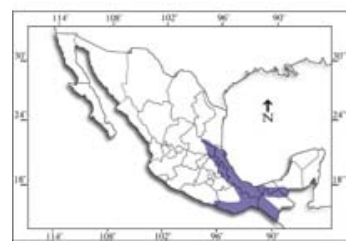

Peromyscus nasutus

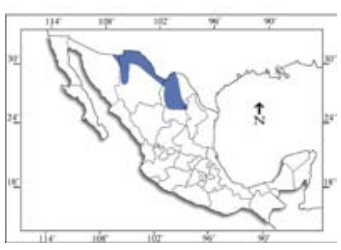


Peromyscus oaxacensis

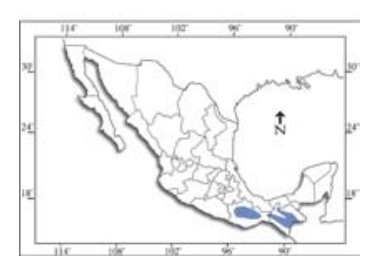

Peromyscus perfulvus

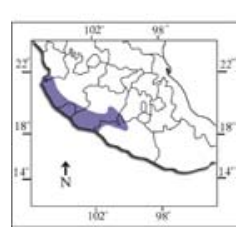

Peromyscus schmidlyi

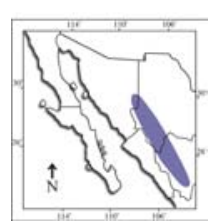

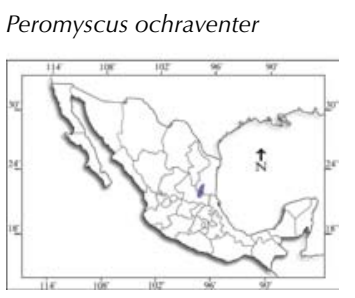

Peromyscus pectoralis

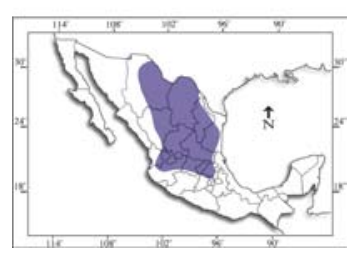

Peromyscus pseudocrinitus
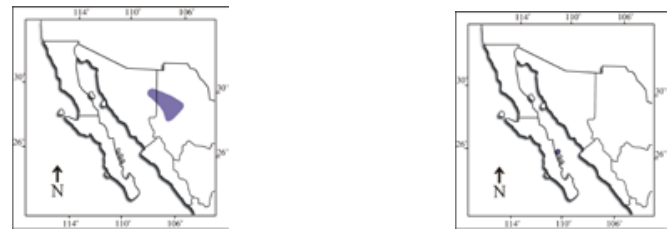

Peromyscus sejugis



Peromyscus simulus

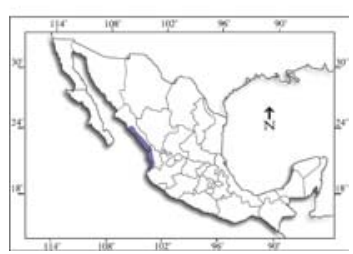

Peromyscus truei

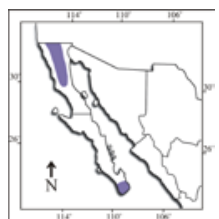

Peromyscus stephani

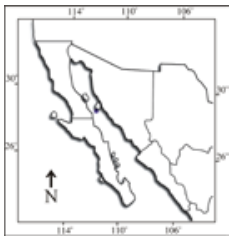

Peromyscus pembertoni

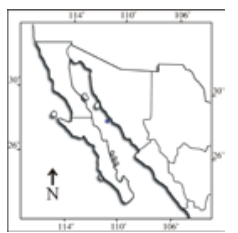

Peromyscus spicilegus

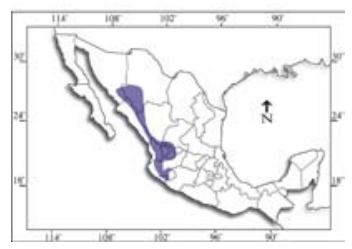

Peromyscus sagax

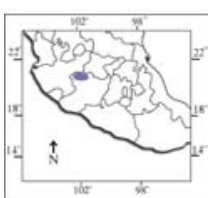

Peromyscus slevini

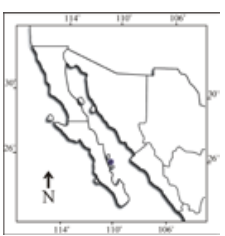

Peromyscus yucatanicus

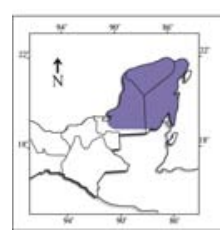

Peromyscus zarhynchus

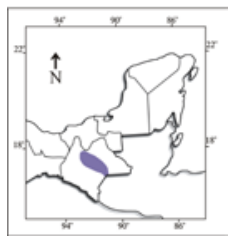

Reithrodontomys bakeri

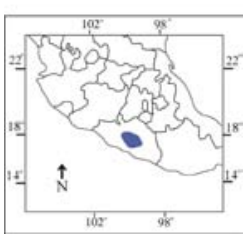

Peromyscus winkelmanni

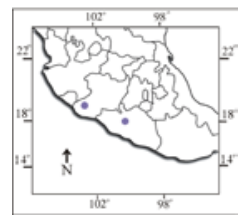



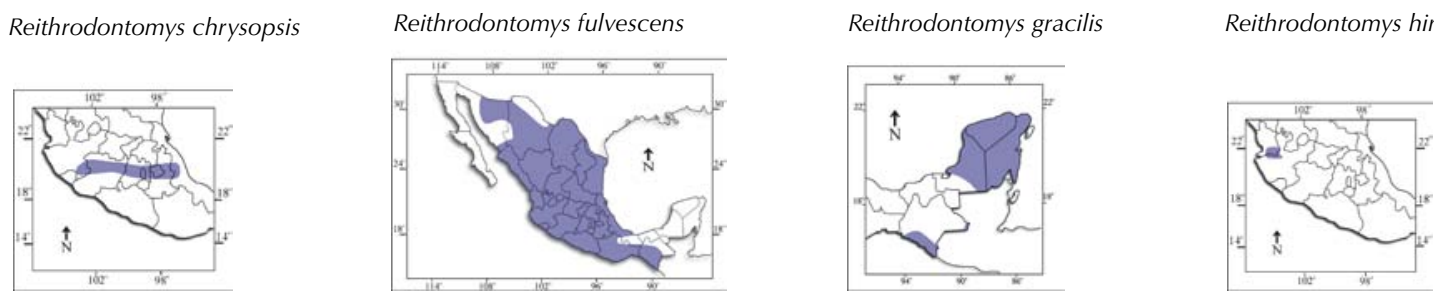

Reithrodontomys megalotis

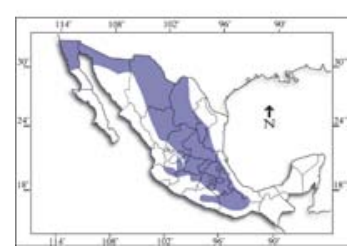

Reithrodontomys spectabilis

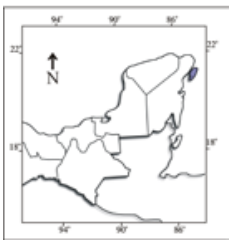

Reithrodontomys mexicanus

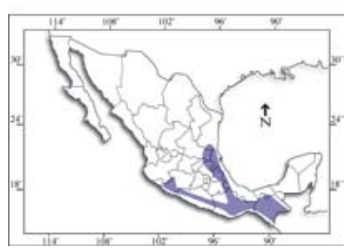

Reithrodontomys microdon

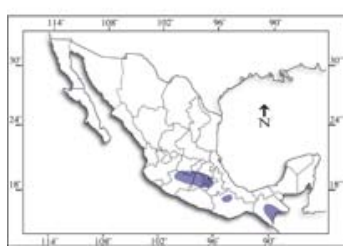

Reithrodontomys montanus

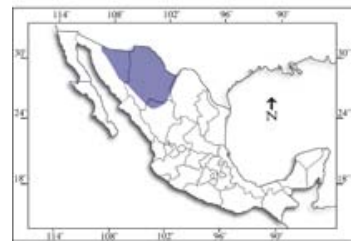

Rheomys mexicanus

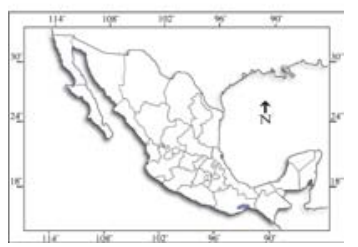

Reithrodontomys sumichrasti

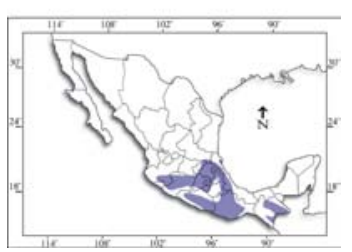

Reithrodontomys tenuirostris

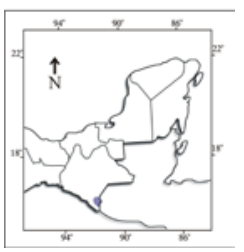

Reithrodontomys zacatecae

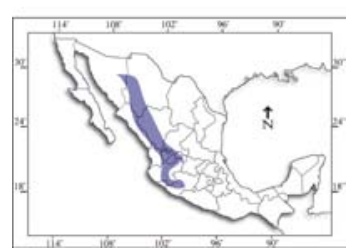

Rheomys thomasi

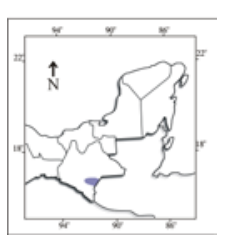

Scotinomys teguina

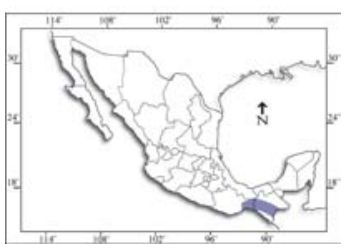

Sigmodon alleni

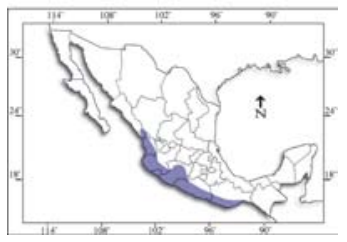

Sigmodon arizonae

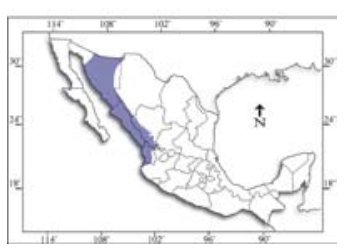

Sigmodon fulviventer

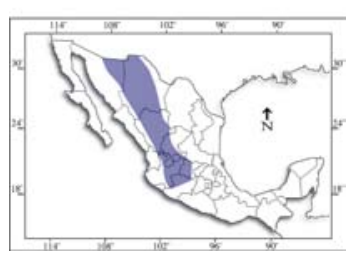

Sigmodon hirsutus

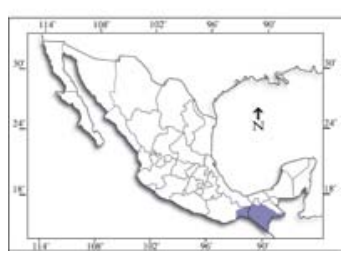

Sigmodon hispidus

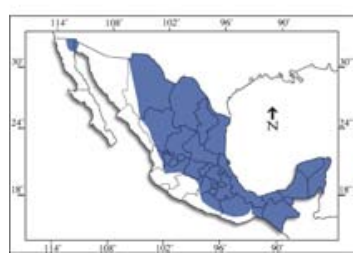


Sigmodon leucotis
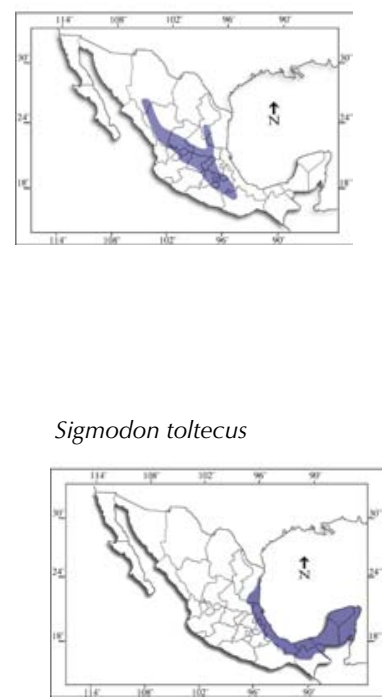

Sigmodon mascotensis

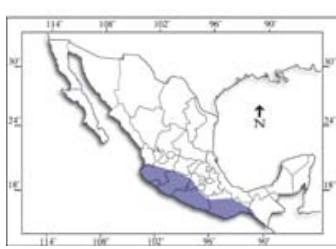

Sigmodon zanjonensis

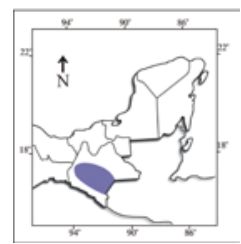

Sigmodon ochrognathus

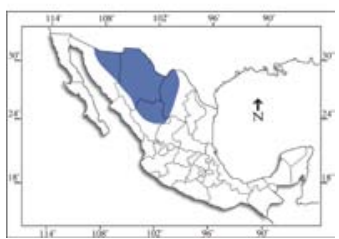

Sigmodon planifrons

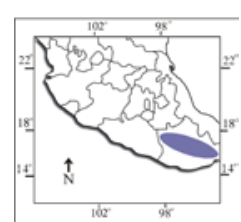

Sigmodon toltecus
Tylomys bullaris

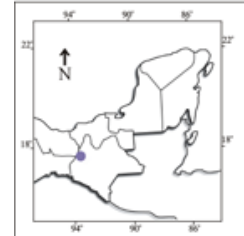

Tylomys nudicaudus

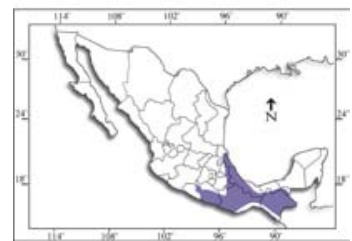

ORDEN RODENTIA

FAMILIA ERETHIZONTIDAE

\section{Tylomys tumbalensis}

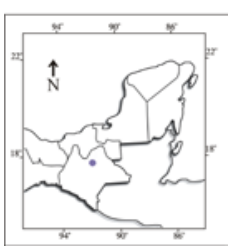

ORDEN RODENTIA

FAMILIA DASYPROCTIDAE

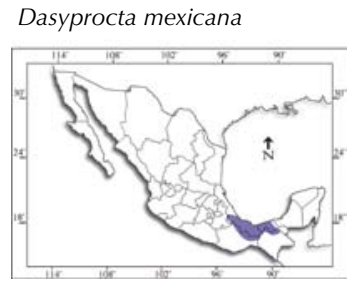

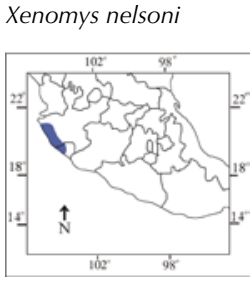

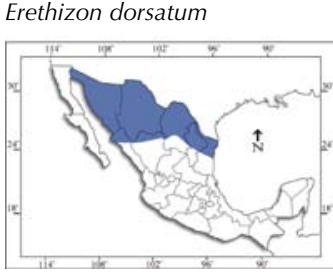

ORDEN RODENTIA

FAMILIA CUNICULIDAE
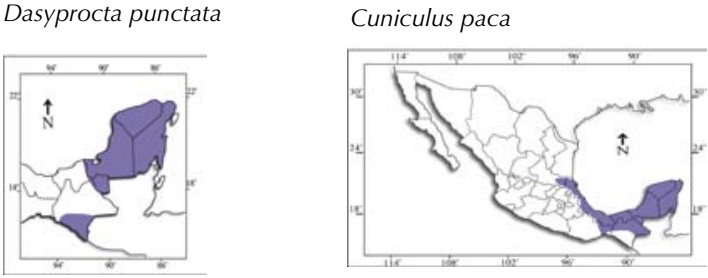

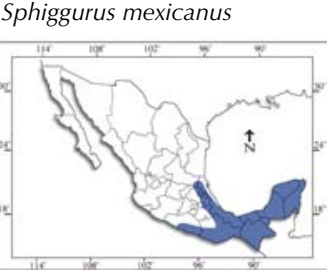

ORDEN SORICOMORPHA

FAMILIA SORICIDAE

Cryptotis alticola

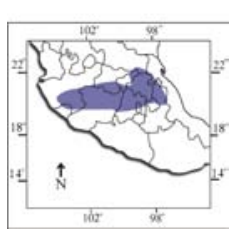

Cryptotis goldmani

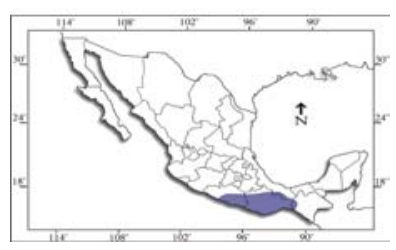

Cryptotis goodwini

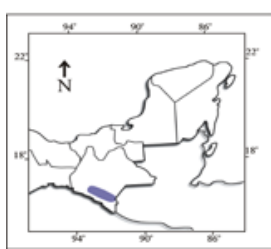

Cryptotis griseoventris

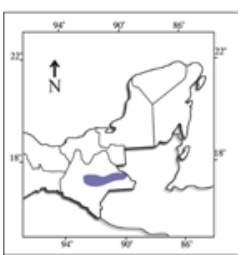

Cryptotis magna

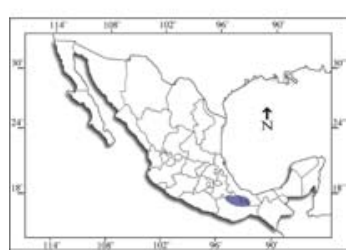


Cryptotis mayensis

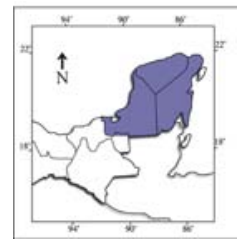

Cryptotis merriami

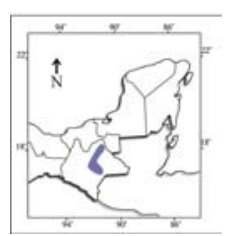

Cryptotis mexicana

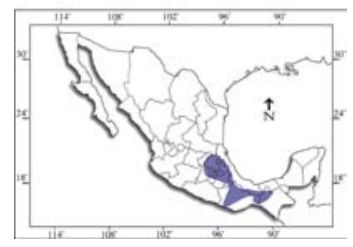

Cryptotis nelsoni

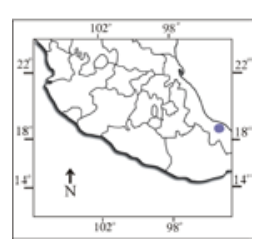

Cryptotis obscura

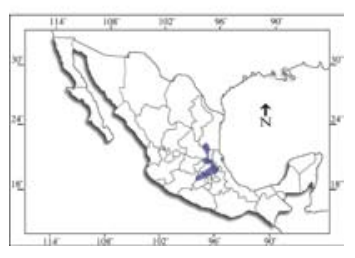

Cryptotis tropicalis

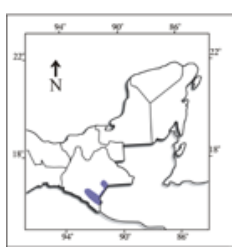

Cryptotis parva

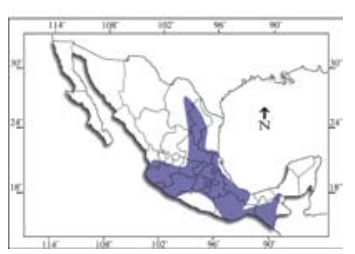

Megasorex gigas

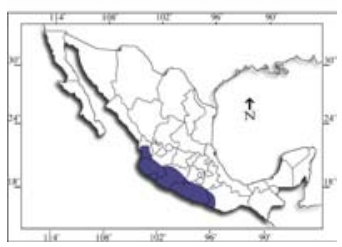

Cryptotis peregrina

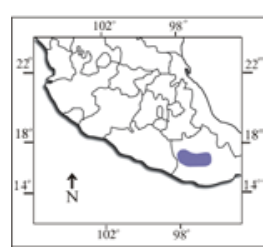

Notiosorex cockrumi

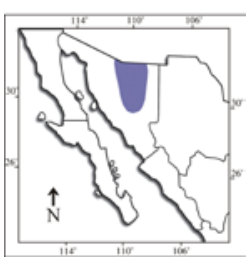

Cryptotis phillipsii

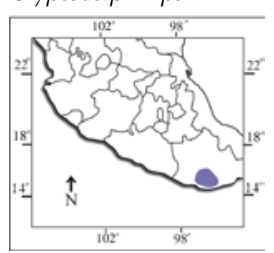

Notiosorex evotis

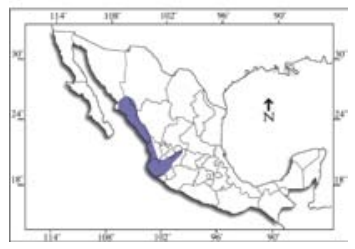

Notiosorex villai

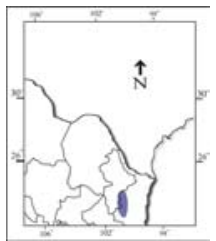

Sorex arizonae

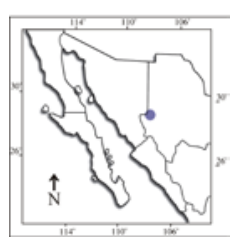

Notiosorex crawfordi

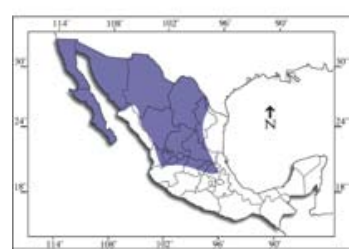

Sorex ixtlanensis

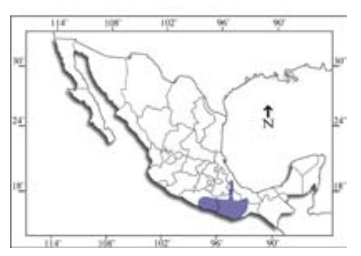

Sorex macrodon

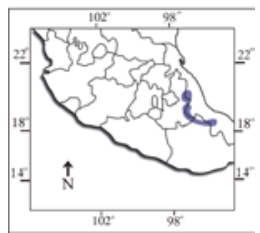

Sorex mediopua

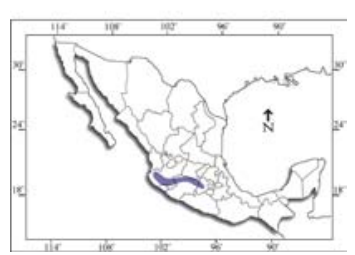

Sorex emarginatus

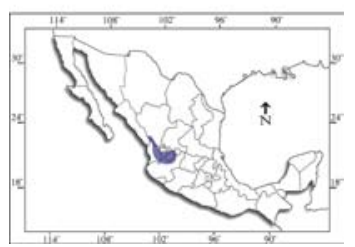

Sorex milleri

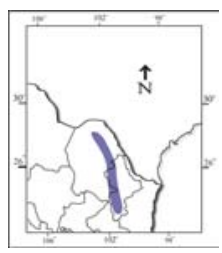


Sorex monticolus

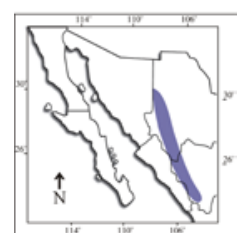

Sorex oreopolus

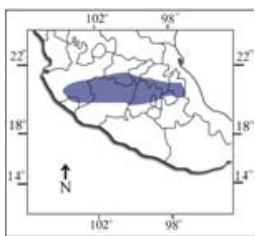

Sorex orizabae

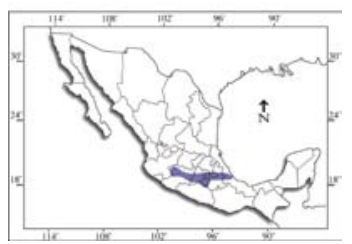

Sorex ornatus

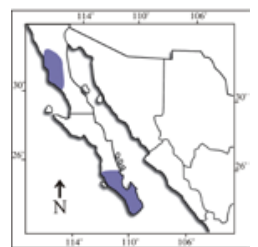

Sorex saussurei

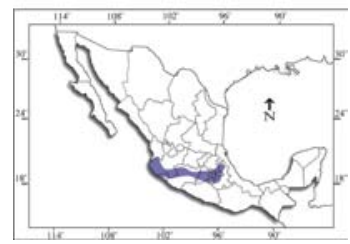

Sorex sclateri

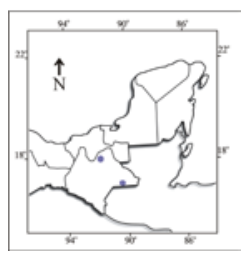

Sorex stizodon

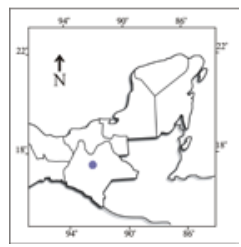

Sorex ventralis

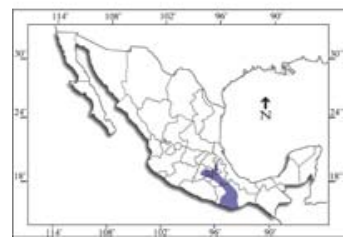

ORDEN SORICOMORPHA

FAMILIA TALPIDAE
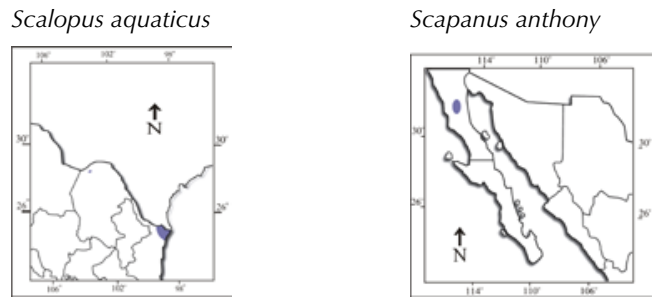

ORDEN CARNIVORA

FAMILIA FELIDAE

Lynx rufus

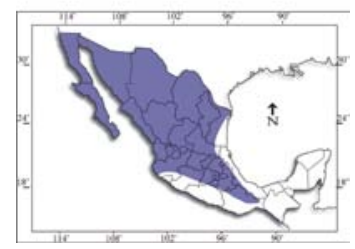

ORDEN CARNIVORA

FAMILIA CANIDAE

Canis latrans

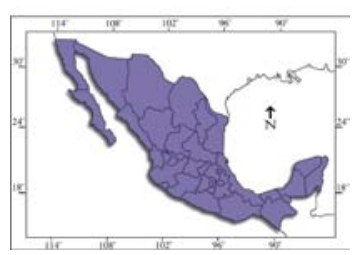

Puma concolor

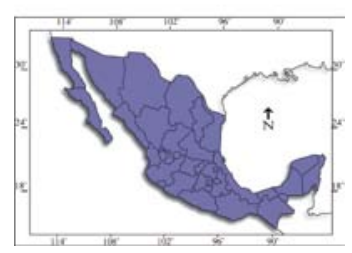

Leopardus wiedii

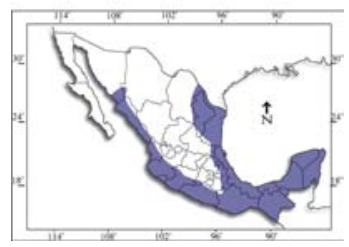

Puma yagouaroundi

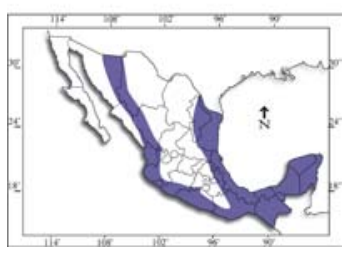

Panthera onca

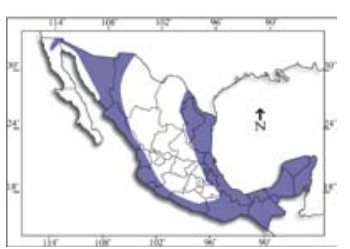

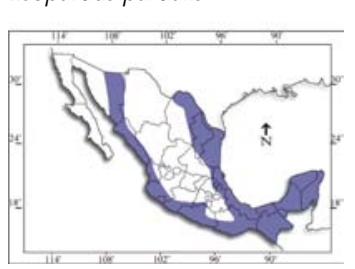

68 • REVISTA MEXICANA DE MASTOZOOLOGÍA Nueva época, 2012, Año 2 Núm. 1 
ORDEN CARNIVORA

FAMILIA URSINAE
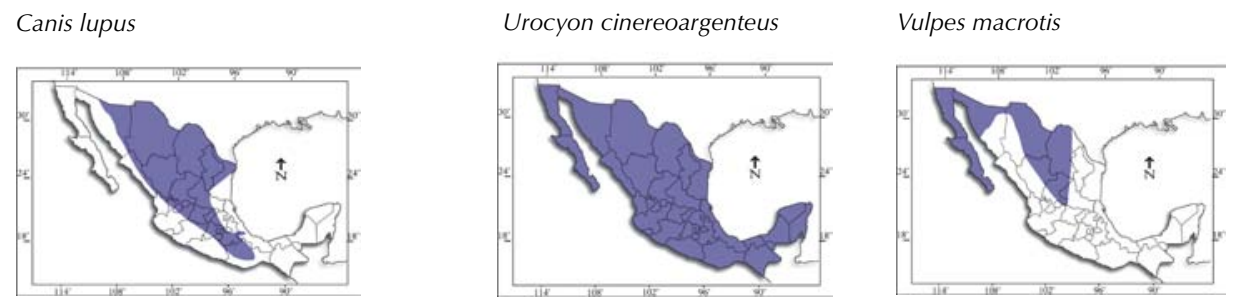

Ursus americanus

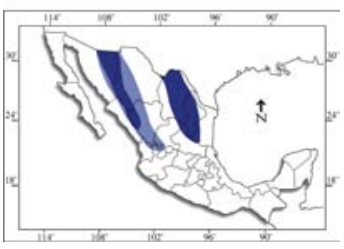

ORDEN CARNIVORA

FAMILIA OTARIIDAE

Ursus arctos

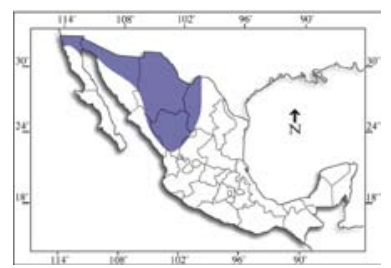

Zalophus californianus

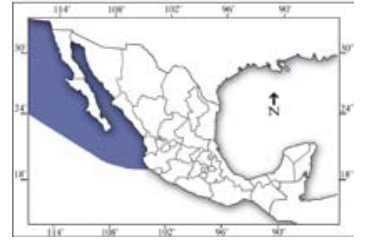

Phoca vitulina

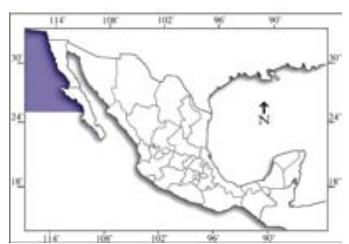

Arctocephalus galapageoensis

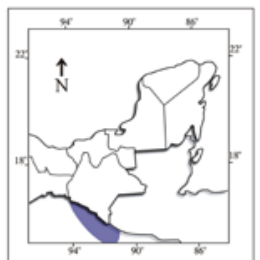

Callorhinus ursinus
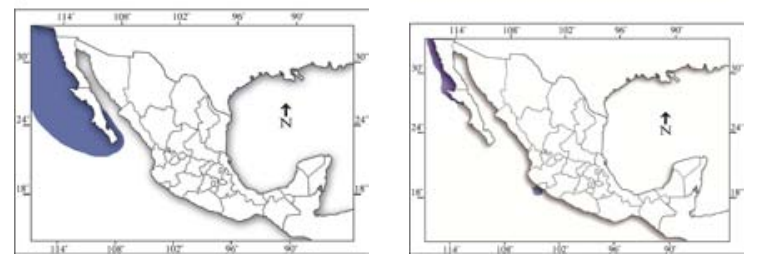

ORDEN CARNIVORA

FAMILIA PHOCIDAE

Monachus tropicalis

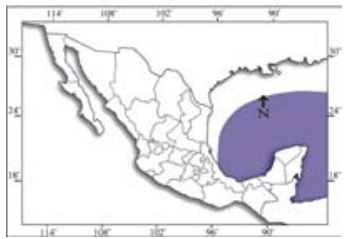

Mirounga angustirostris

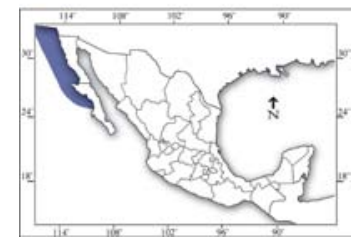

\section{ORDEN CARNIVORA}

FAMILIA MUSTELIDAE

Enhydra lutris

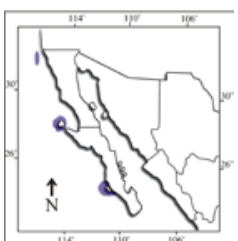

Lontra canadensis

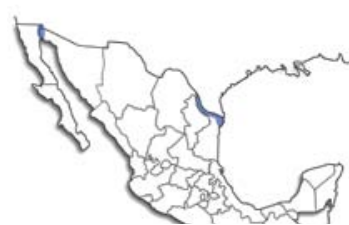

Lontra longicaudis

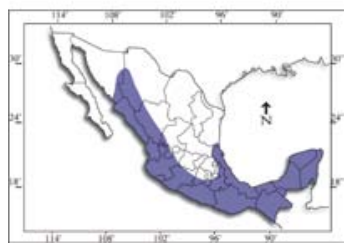

Eira barbara

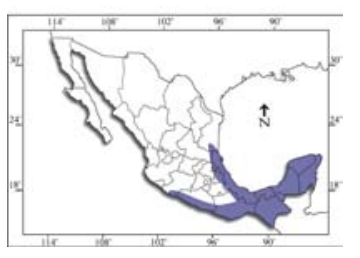

Galictis vittata

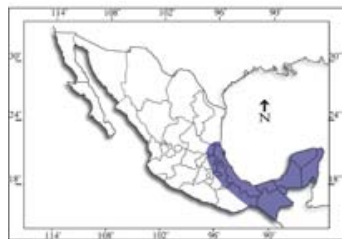

Mustela frenata

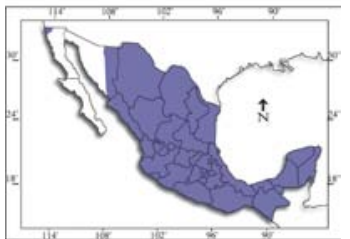

Mustela nigripes

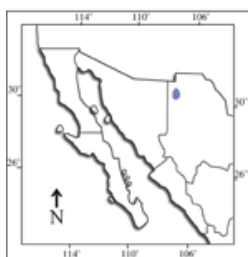




\section{ORDEN CARNIVORA}

FAMILIA MEPHITINAE

Taxidea taxus

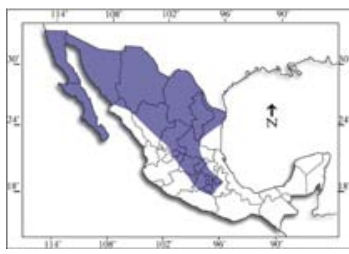

Conepatus leuconotus

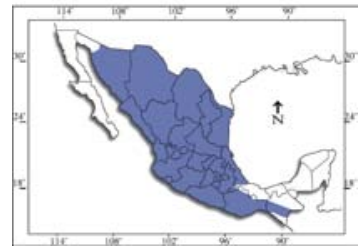

Conepatus semistriatus

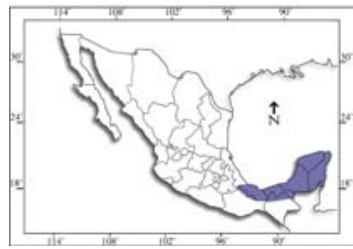

Mephitis macroura

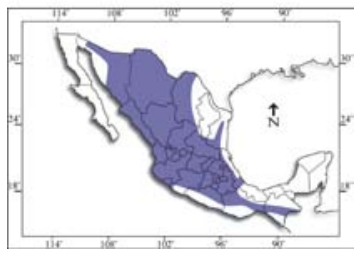

Mephitis mephitis

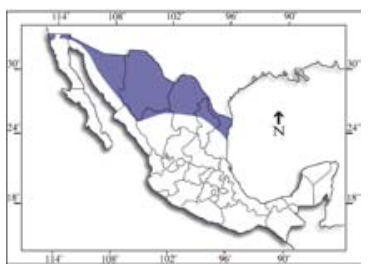

Spilogale angustifrons

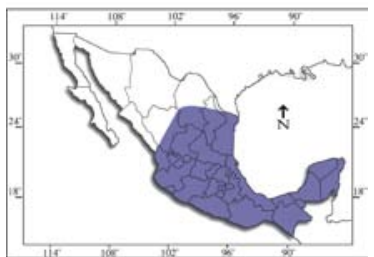

Spilogale gracilis

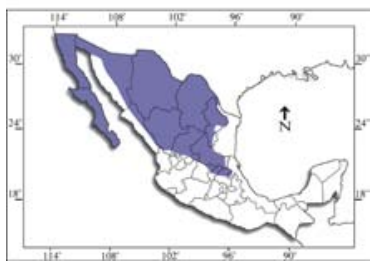

Spilogale pygmaea

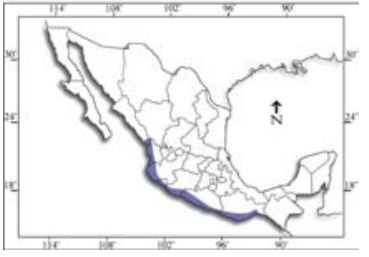

ORDEN CARNIVORA

FAMILIA PROCYONIDAE
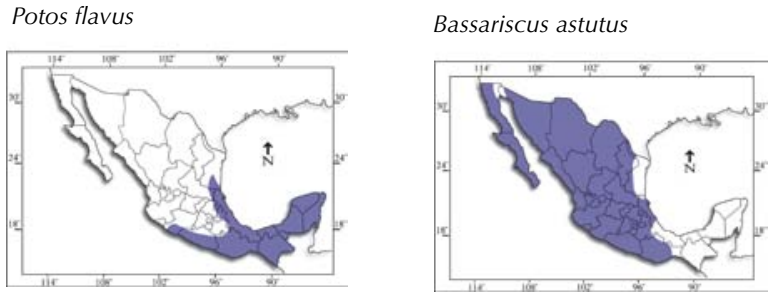

Bassariscus sumichrasti

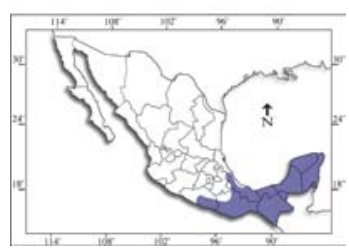

Nasua narica

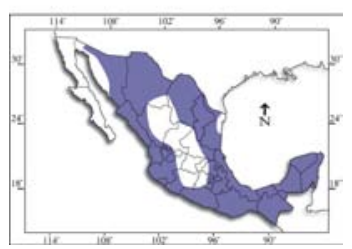

ORDEN PERISSODACTYLA

FAMILIA TAPIRIDAE

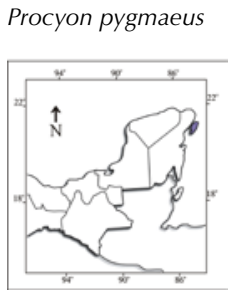

Tapirus bairdii

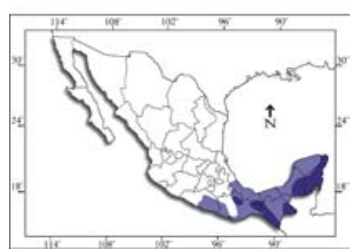

ORDEN ARTIODACTYLA

FAMILIA TAYASSUIDAE

Pecari tajacu

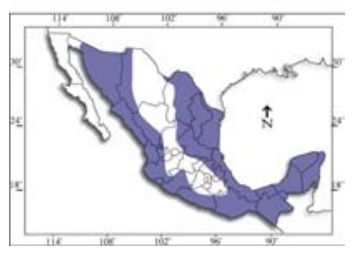

Procyon lotor

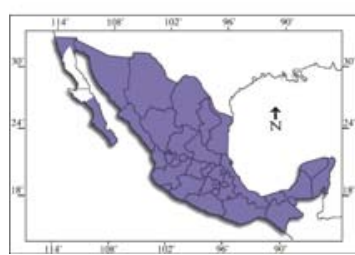

ORDEN ARTIODACTYLA

FAMILIA CERVIDAE

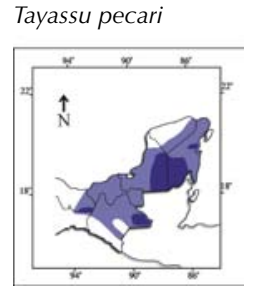

Cervus canadensis

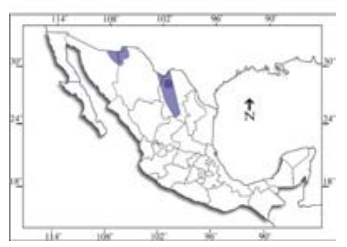

Mazama americana

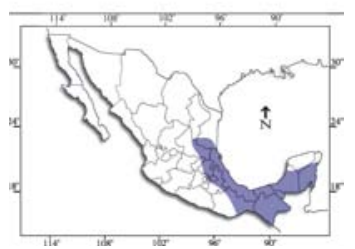


ORDEN ARTIODACTYLA

FAMILIA ANTILOCAPRIDAE

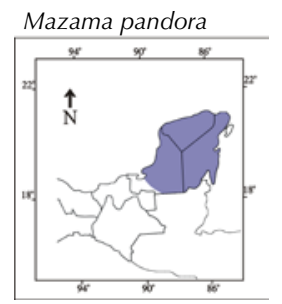

Odocoileus hemionus

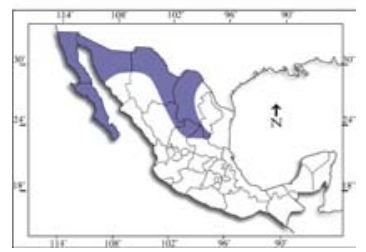

ORDEN ARTIODACTYLA

FAMILIA BOVIDAE

Bison bison

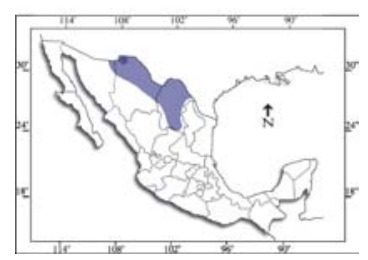

Balaenoptera borealis

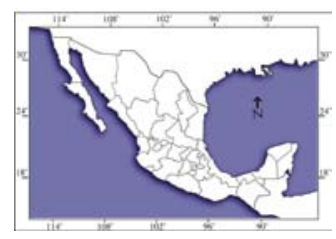

Megaptera novaeangliae

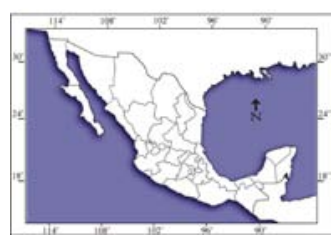

Physeter macrocephalus

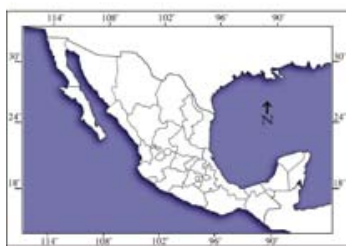

ORDEN CETACEA

FAMILIA ESCHRICHTIDAE

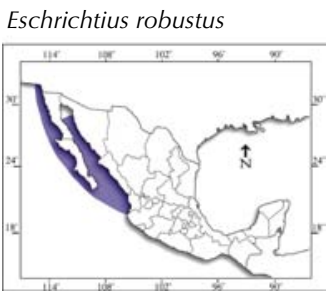

Berardius bairdi

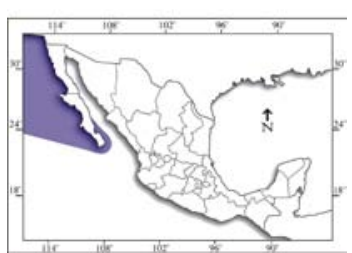

Odocoileus virginianus

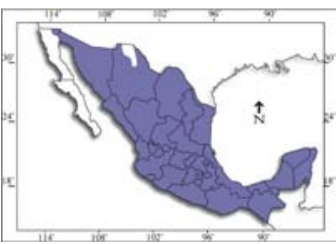

ORDEN CETACEA

FAMILIA BALAENIDAE

Eubalaena japonica

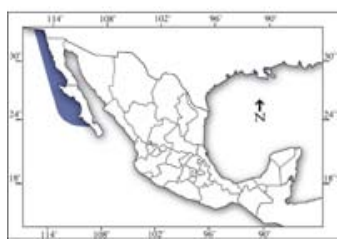

Balaenoptera musculus

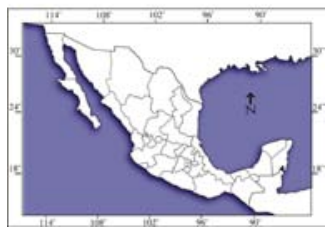

SUBORDEN ODONTOCETI

FAMILIA PHYSETERIDAE

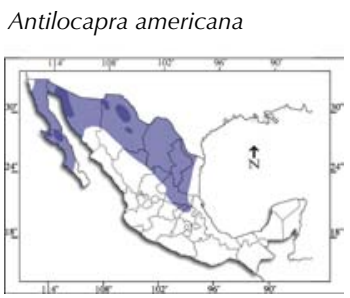

ORDEN CETACEA

FAMILIA BALAENOPTERIDAE Balaenoptera acutorostrata

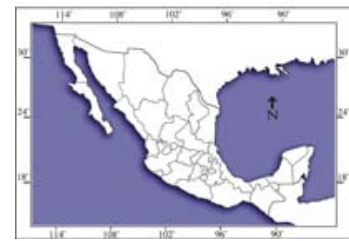

Balaenoptera physalus

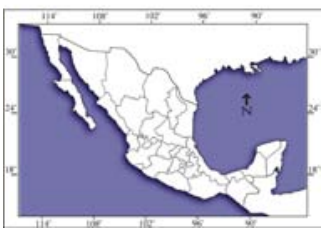

Kogia breviceps

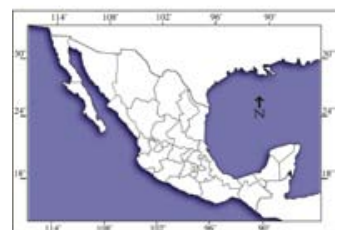

Kogia sima

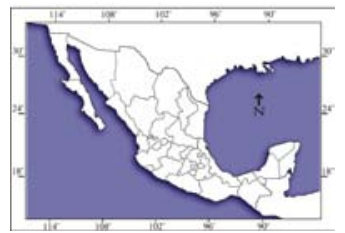

SUBORDEN ODONTOCETI

FAMILIA ZIPHIIDAE
Indopacetus pacificus

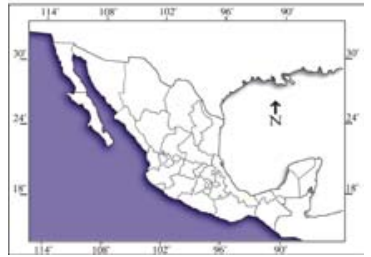

Mesoplodon carlhubbsi

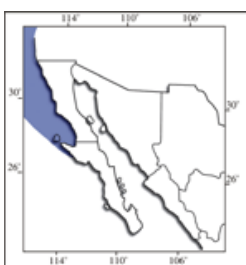


Mesoplodon densirostris
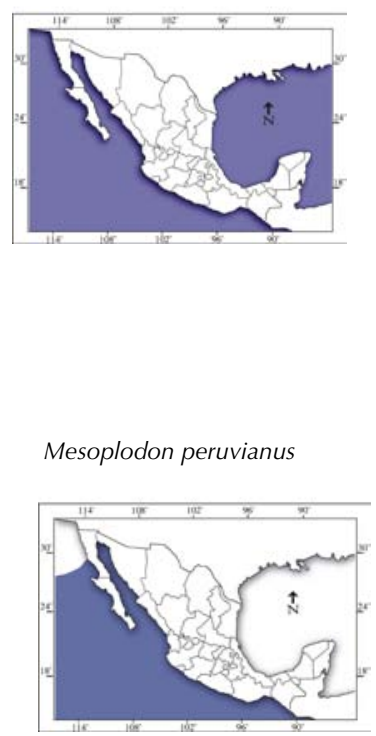

Feresa attenuata

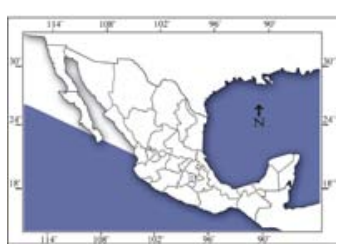

Lagenorhynchus obliquidens

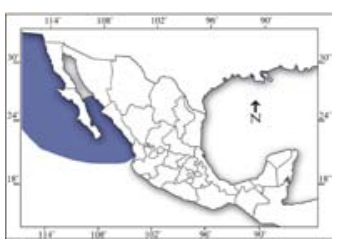

Pseudorca crassidens

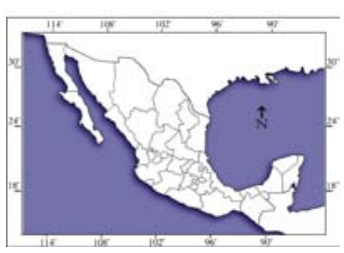

Mesoplodon europaeus

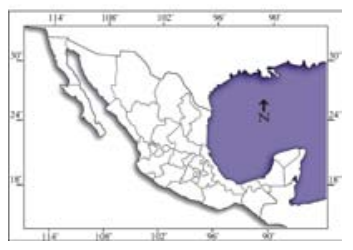

Ziphius cavirostris

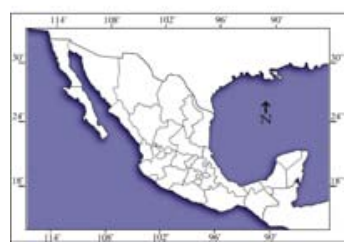

Globicephala macrorhynchus

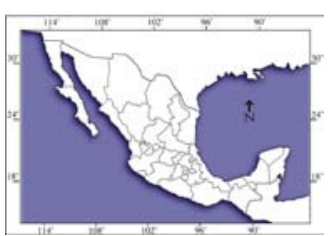

Grampus griseus

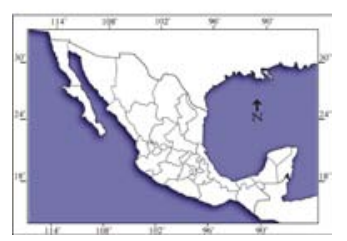

FAMILIA DELPHINIDAE
Delphinus capensis

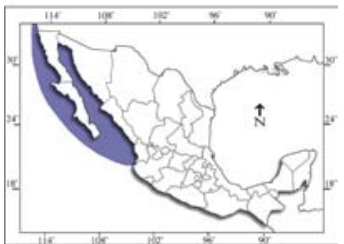

Mesoplodon perrini

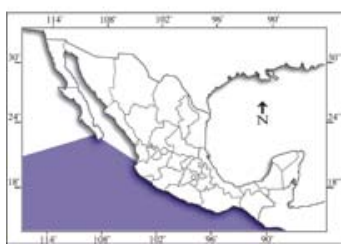

SUBORDEN ODONTOCETI

Lagenodelphis hosei

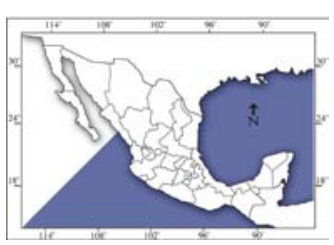

Peponocephala electra
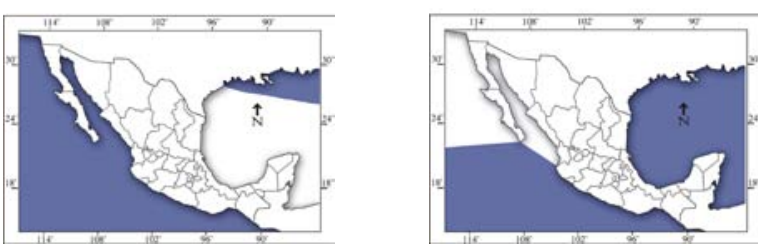

Stenella coeruleoalba
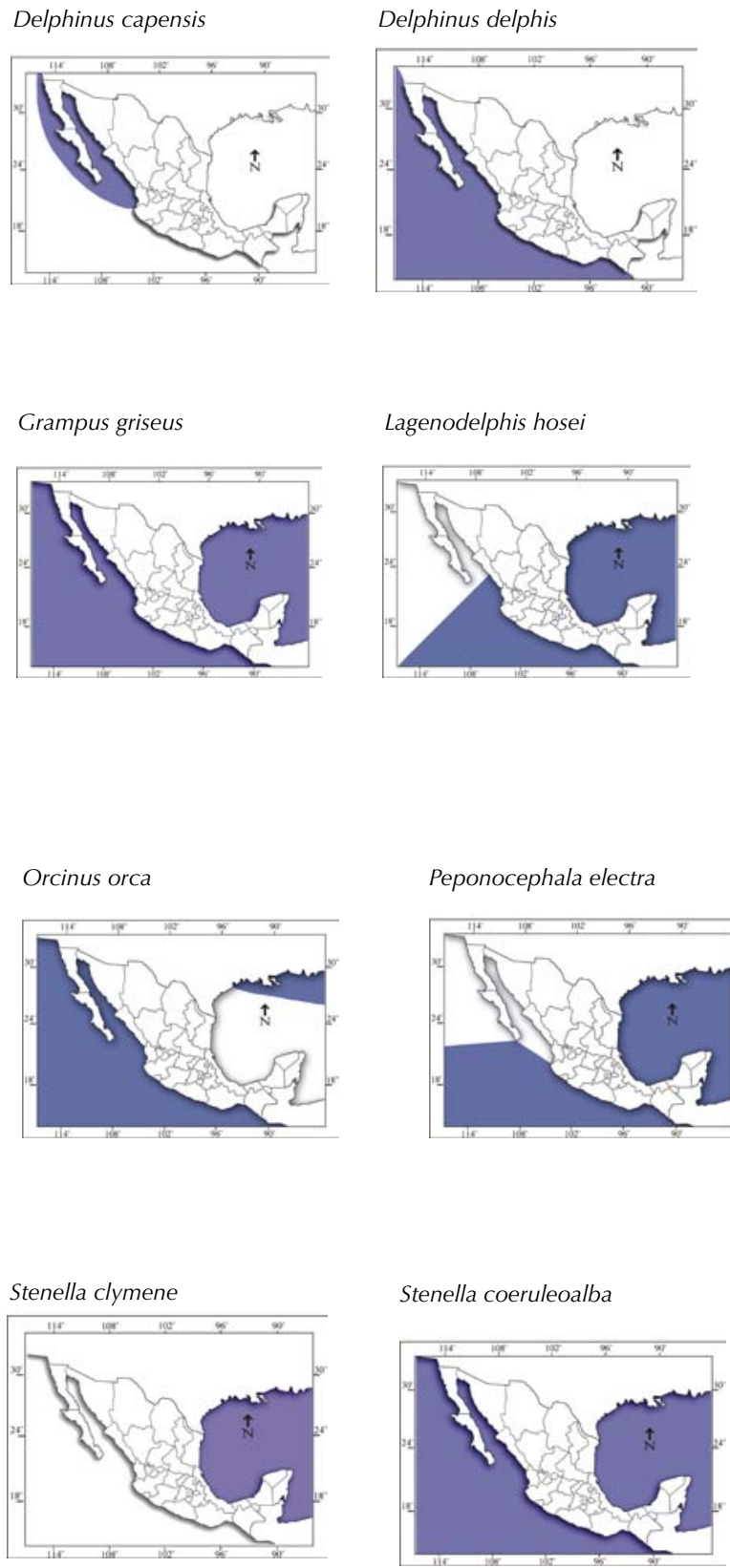

Lissodelphis borealis

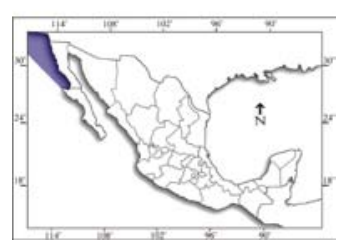

Stenella attenuata

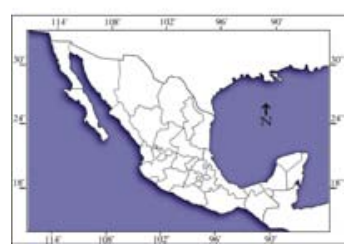


Stenella frontalis

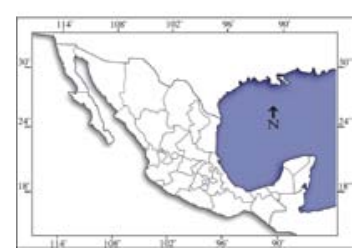

SUBORDEN ODONTOCETI

FAMILIA PHOCOENIDAE

Phocoena sinus

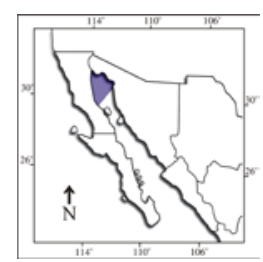

Centronycteris centralis

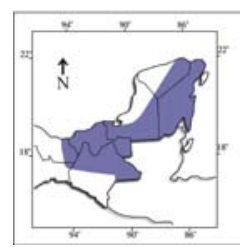

Stenella longirostris

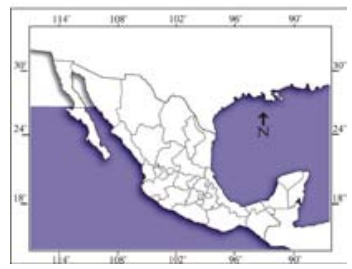

Steno bredanensis

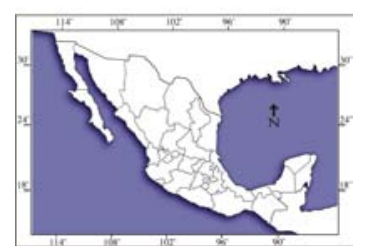

Tursiops truncatus

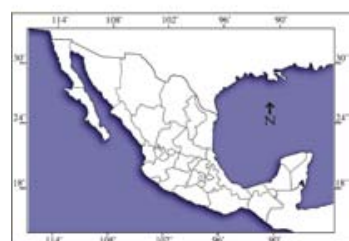

\section{ORDEN CHIROPTERA}

FAMILIA EMBALLONURIDAE

Phocoenoides dalli

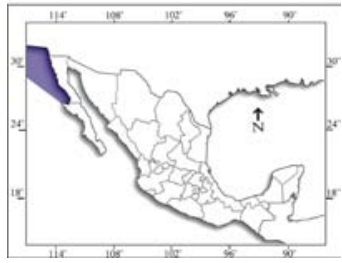

Balantiopteryx io

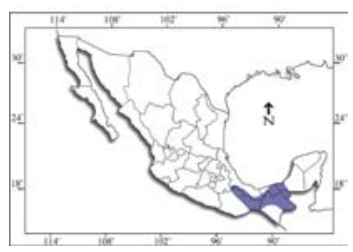

Balantiopteryx plicata

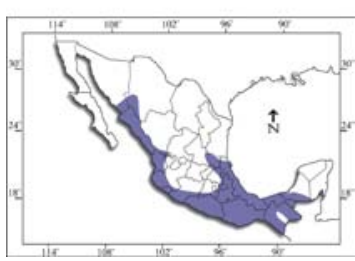

Diclidurus albus

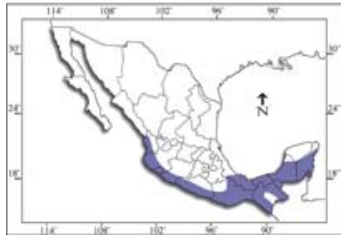

Saccopteryx bilineata

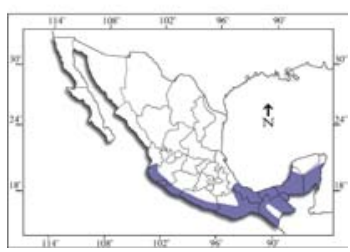

Glyphonycteris sylvestris

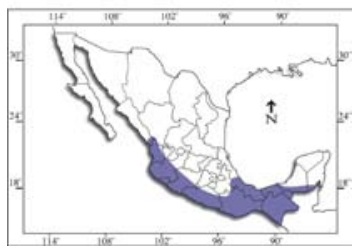

Peropteryx kappleri

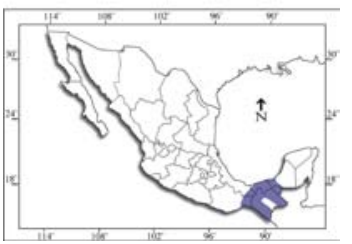

ORDEN CHIROPTERA

FAMILIA PHYLLOSTOMIDAE

Macrotus californicus

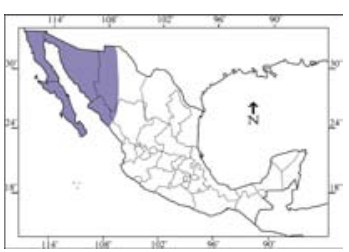

Peropteryx macrotis

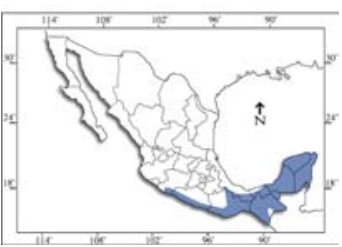

Macrotus waterhousii

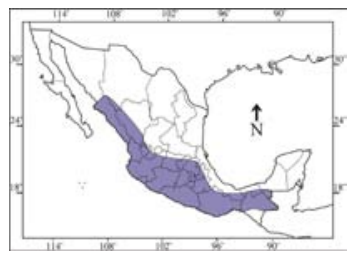

Saccopteryx leptura

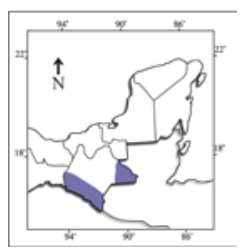

Lampronycteris brachyotis

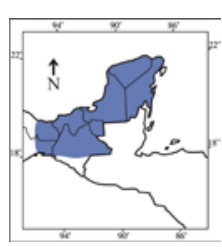

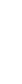

Rynchonycteris naso
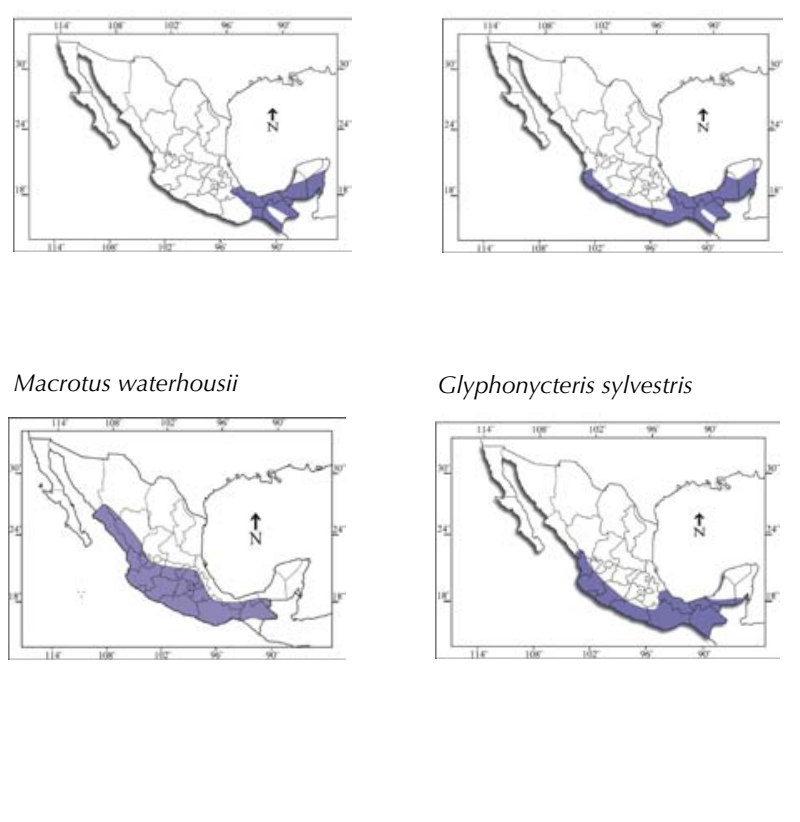
Micronycteris schmidtorum

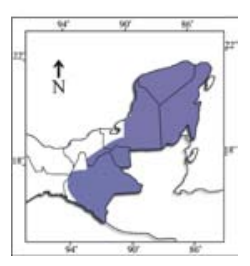

Trinycteris nicefori

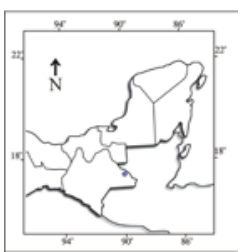

Desmodus rotundus

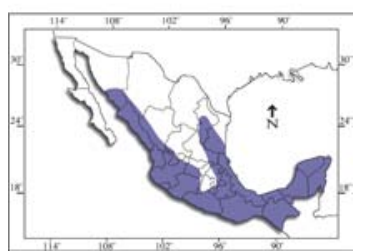

Diaemus youngi

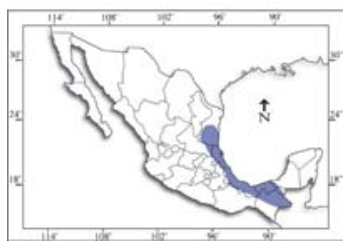

Diphylla ecaudata

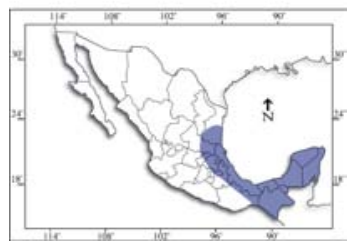

Chrotopterus auritus

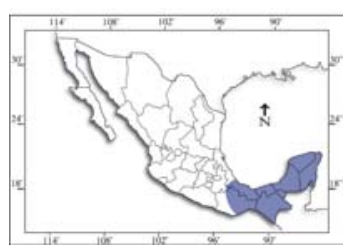

Trachops cirrhosus

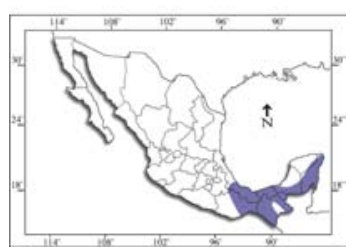

Vampyrum spectrum

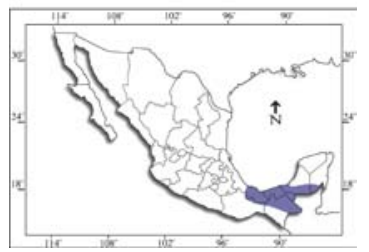

\section{ORDEN CHIROPTERA}

TRIBU PHYLLOSTOMINI

Lophostoma brasiliense

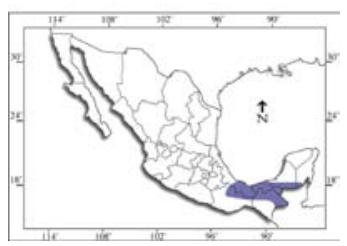

Lophostoma evotis

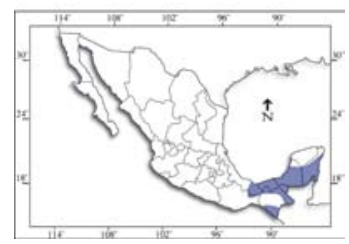

Macrophyllum macrophyllum

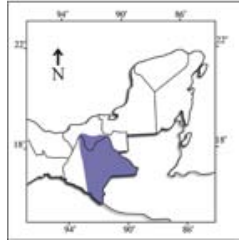

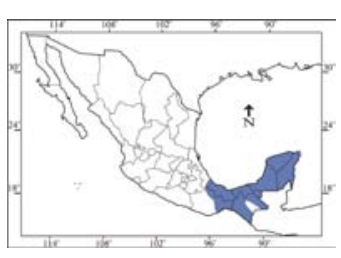

Mimon crenulatum

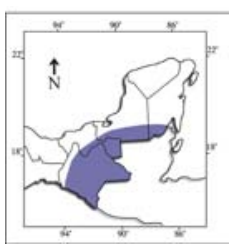

Phylloderma stenops

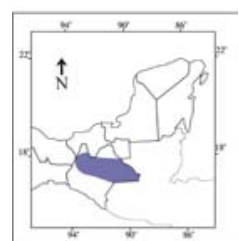

Phyllostomus discolor

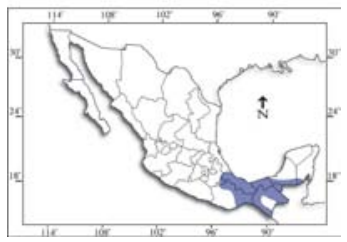

\section{ORDEN CHIROPTERA}

TRIBU GLOSSOPHAGINI

Tonatia saurophila

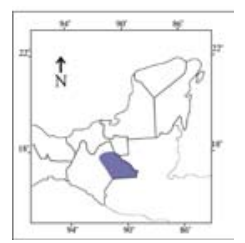

Anoura geoffroyi

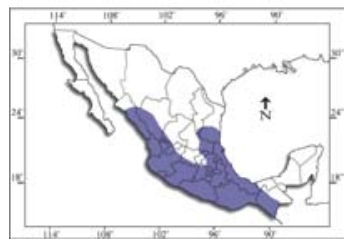

Choeroniscus godmani

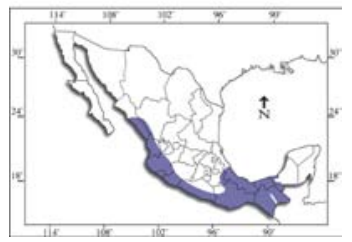

Choeronycteris mexicana

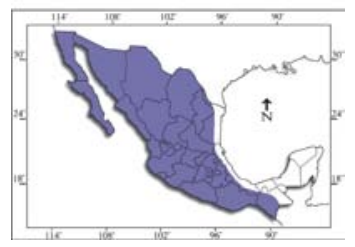


Glossophaga commissarisi

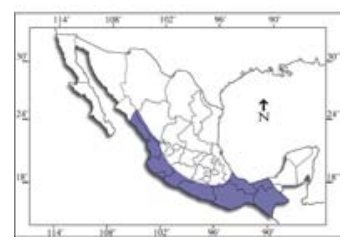

Hylonycteris underwoodi
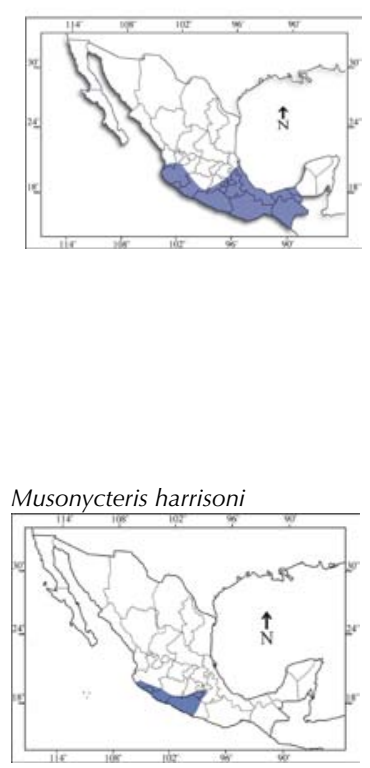

Carollia perspicillata

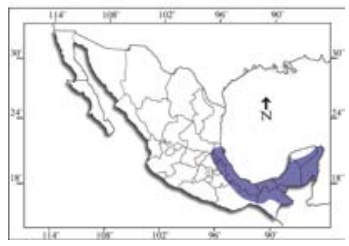

Chiroderma salvini

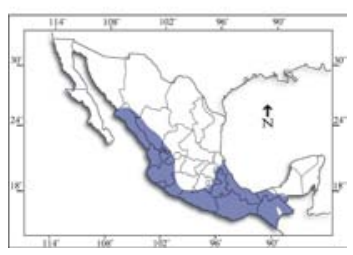

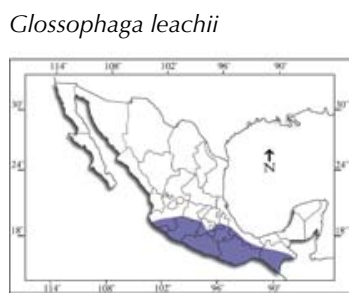

Glossophaga morenoi

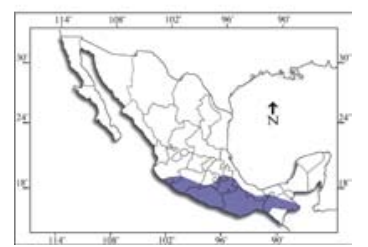

Glossophaga soricina

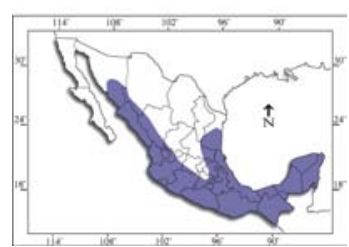

Lichonycteris obscura

Leptonycteris yerbabuenae
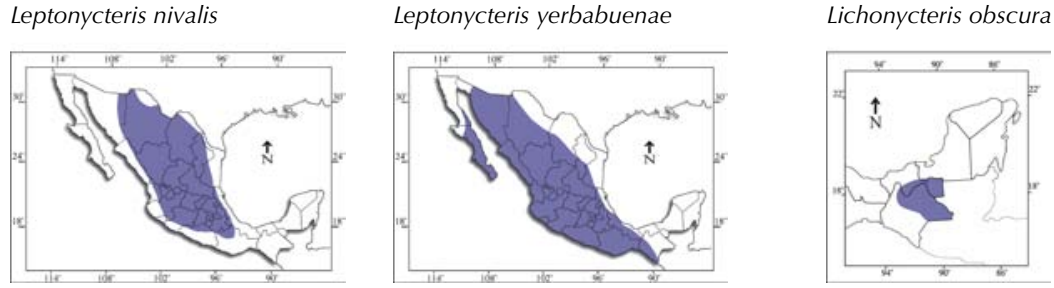

\section{ORDEN CHIROPTERA}

TRIBU STENODERMATINI

Artibeus hirsutus

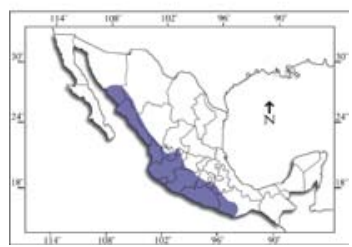

Artibeus jamaicensis

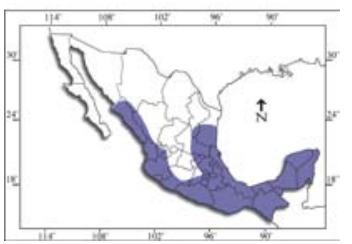

Artibeus lituratus

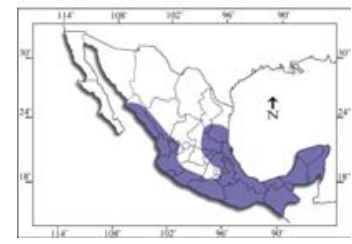

Carollia sowelli

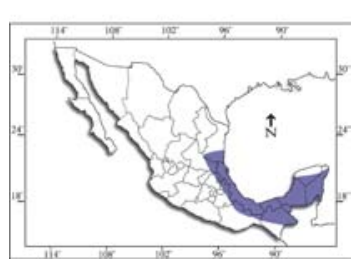

Carollia subrufa

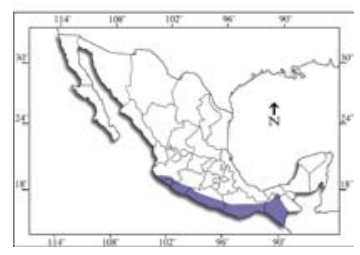

Centurio senex

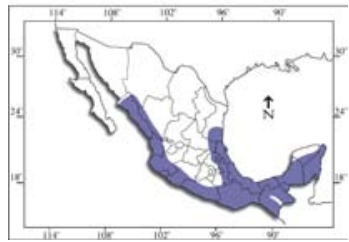

Chiroderma villosum

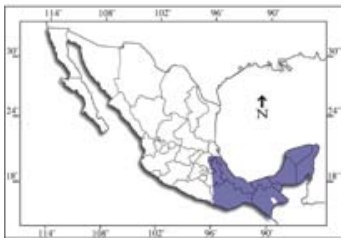

Dermanura azteca

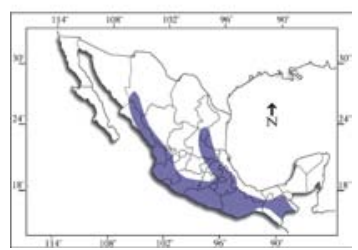

Dermanura phaeotis

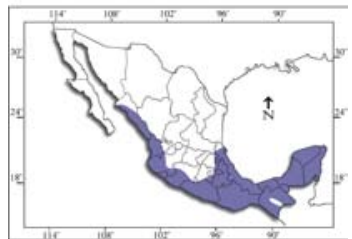




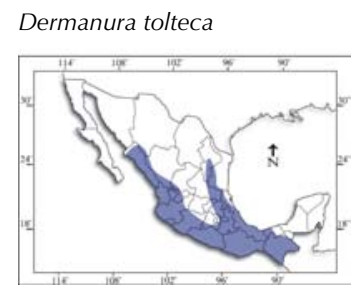

Dermanura watsoni

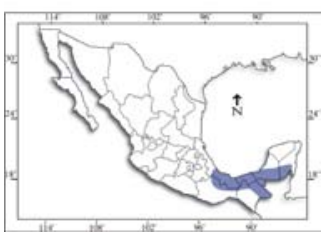

Enchisthenes hartii

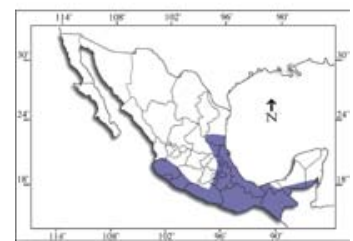

Platyrrhinus helleri

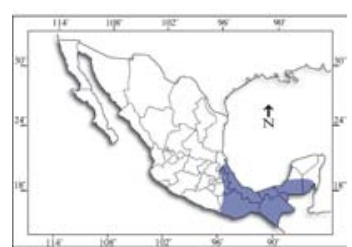

Sturnira hondurensis

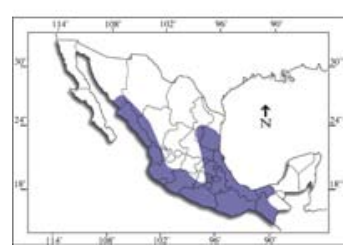

Vampyressa thyone

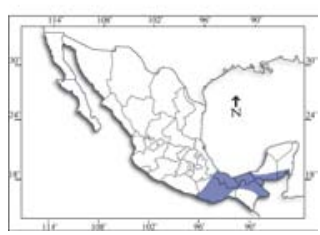

Pteronotus gymnonotus

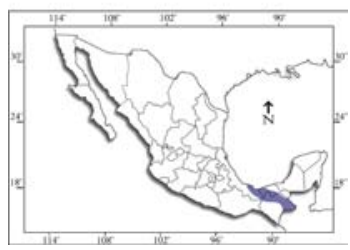

Noctilio leporinus

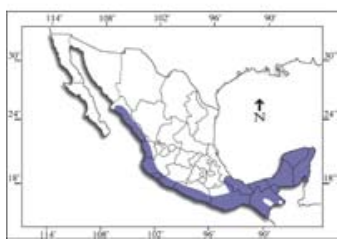

Sturnira lilium

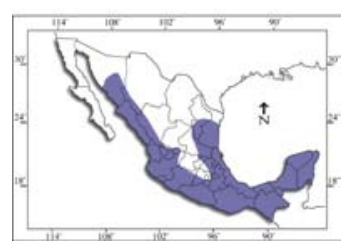

Vampyrodes major

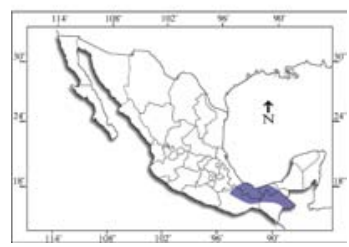

Pteronotus parnellii

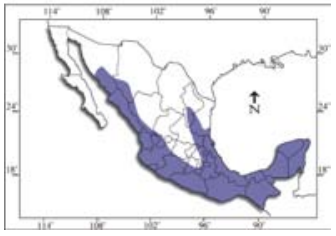

ORDEN CHIROPTERA

FAMILIA THYROPTERIDAE

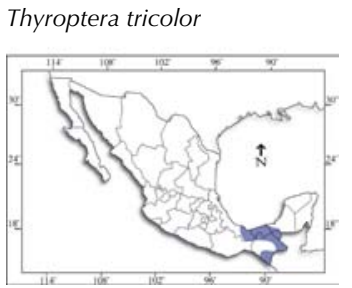

Uroderma bilobatum

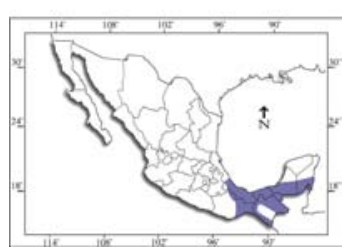

\section{ORDEN CHIROPTERA}

FAMILIA MORMOOPIDAE

Mormoops megalophylla

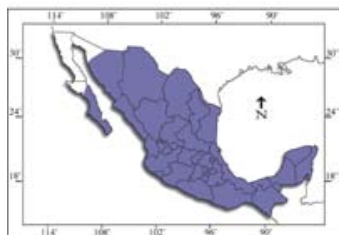

ORDEN CHIROPTERA

FAMILIA NOCTILIONIDAE

Pteronotus personatus

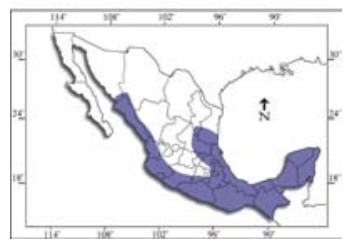

Noctilio albiventris

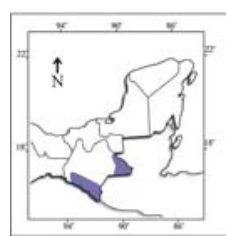

Uroderma magnirostrum

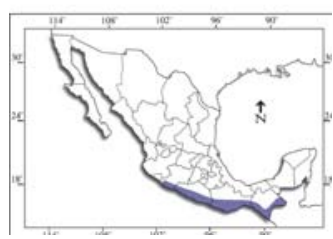

Pteronotus davyi

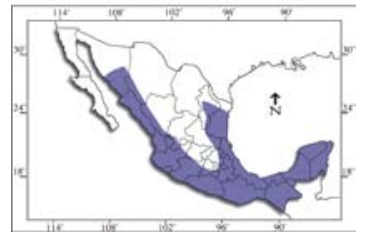

\section{ORDEN CHIROPTERA}

FAMILIA NATALIDAE

Natalus mexicanus

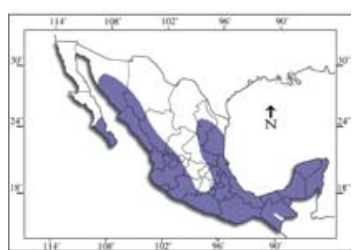


ORDEN CHIROPTERA

FAMILIA MOLOSSIDAE

Eumops auripendulus

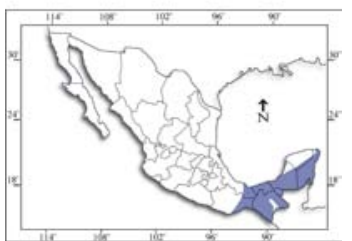

Eumops perotis

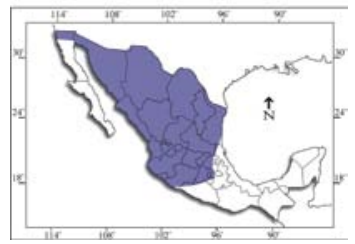

Molossus coibensis

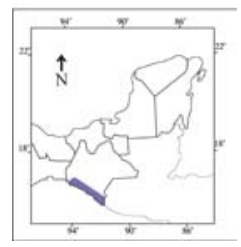

Nyctinomops aurispinosus

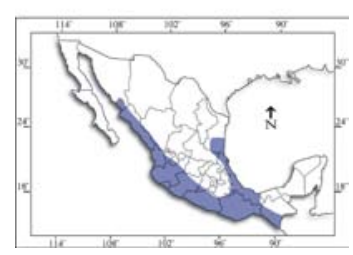

Promops centralis

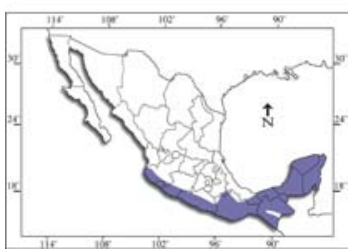

Eumops ferox

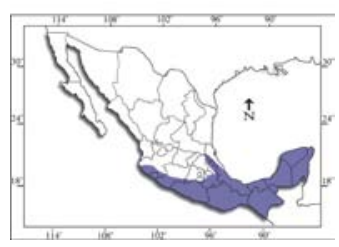

Eumops hansae

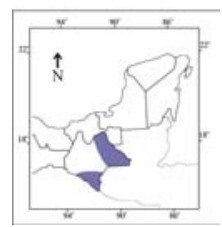

Eumops underwoodi

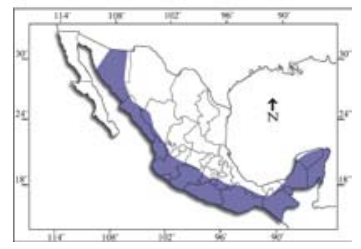

Molossus alvarezi

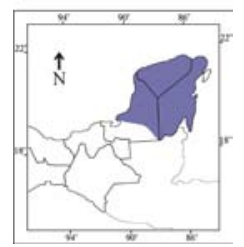

Eumops nanus

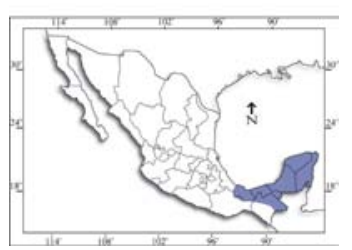

Molossus aztecus

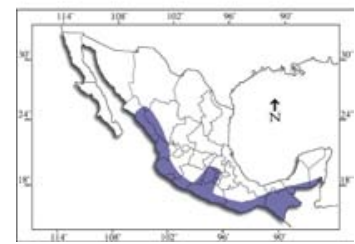

Molossus molossus

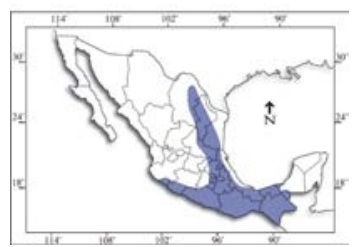

Nyctinomops femorosaccus

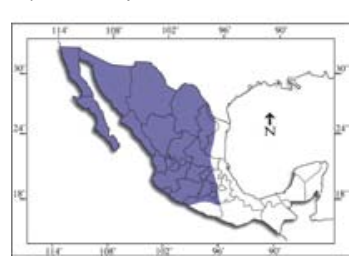

Nyctinomops laticaudatus

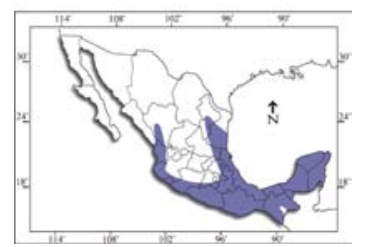

Molossus sinaloae

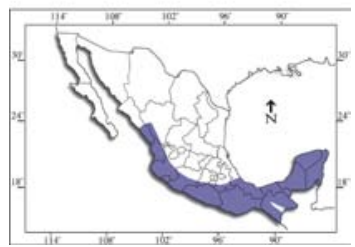

Nyctinomops macrotis

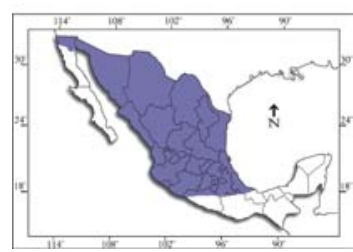

ORDEN CHIROPTERA

FAMILIA VESPERTILIONIDAE

Myotis auriculus

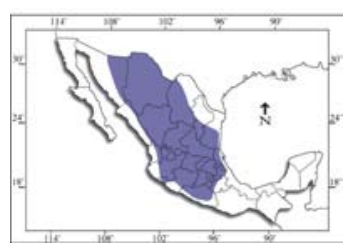



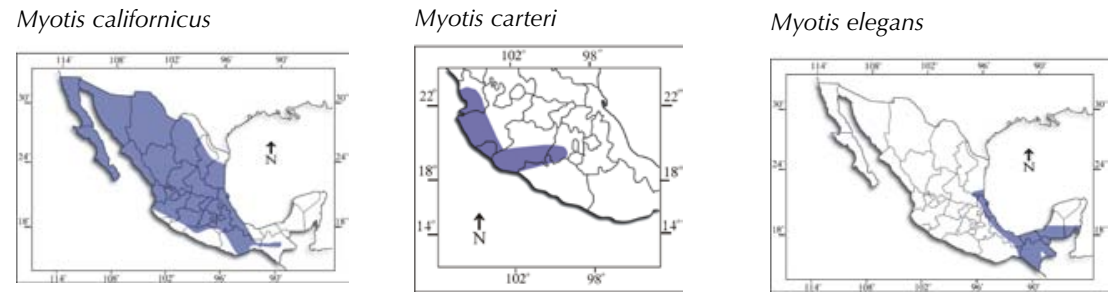

Myotis evotis

Myotis findleyi

Myotis fortidens

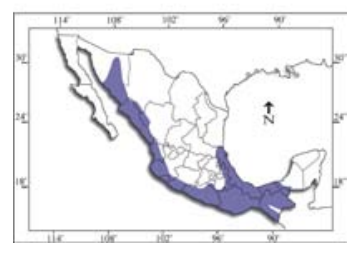

Myotis keaysi

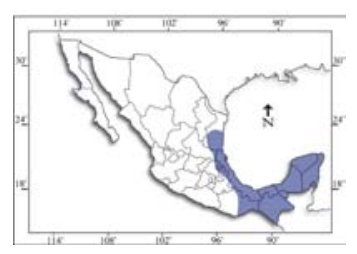

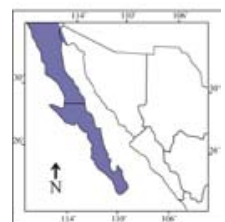

Myotis melanorhinus

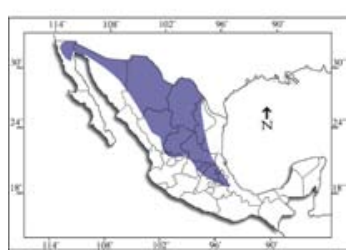

Myotis nigricans

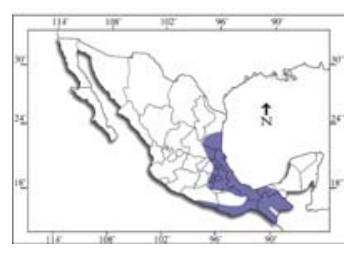

Myotis thysanodes

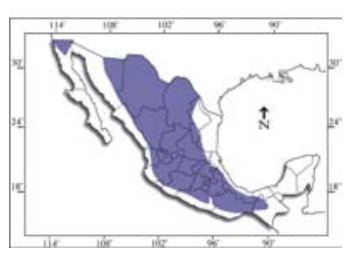

Myotis occultus

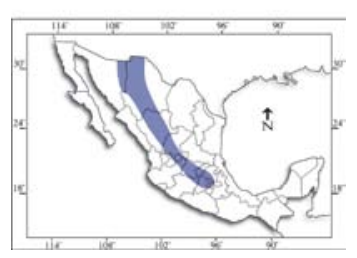



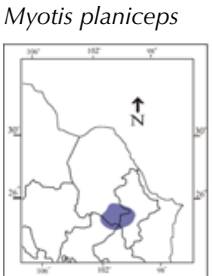

Myotis yumanensis

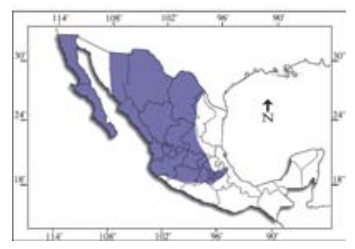

Myotis velifer

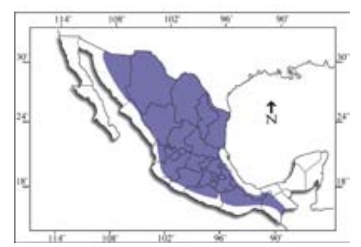

Myotis vivesi

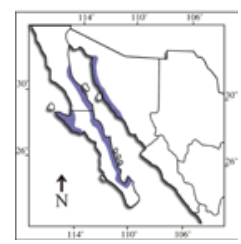

Myotis volans

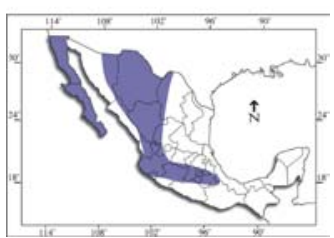

Baeodon alleni

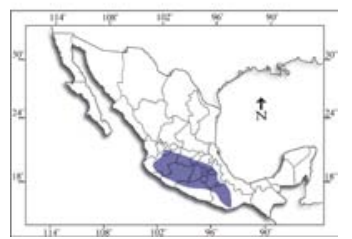

Baeodon gracilis

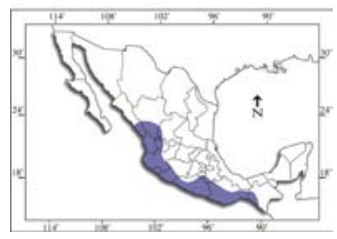

Corynorhinus mexicanus

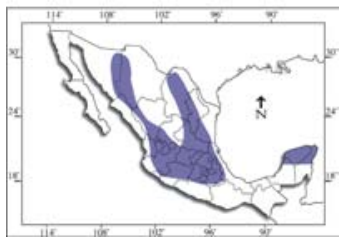




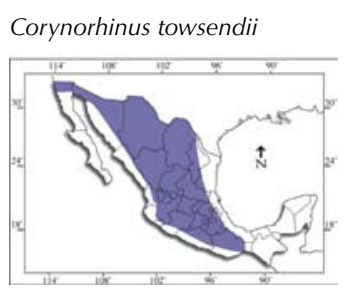

Eptesicus brasiliensis

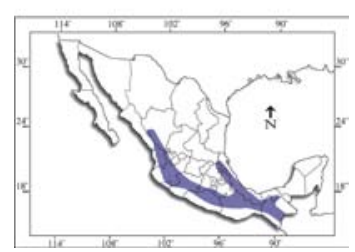

Eptesicus furinalis

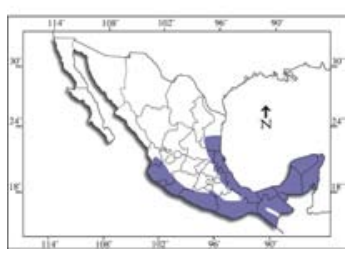

Lasionycteris noctivagans

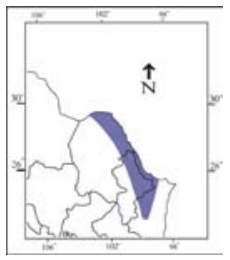

Idionycteris phyllotis

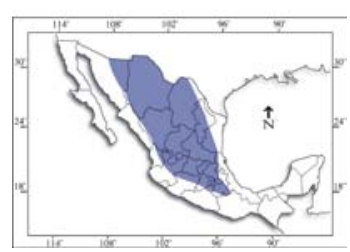

Eptesicus fuscus

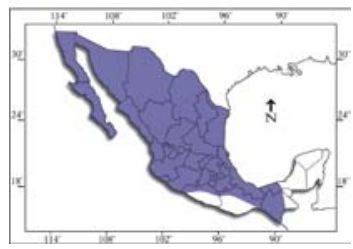

Euderma maculatum

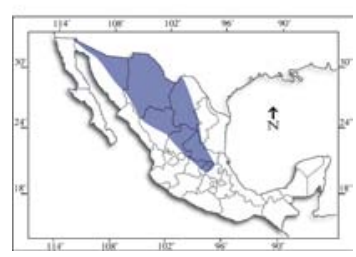

Lasiurus blossevillii

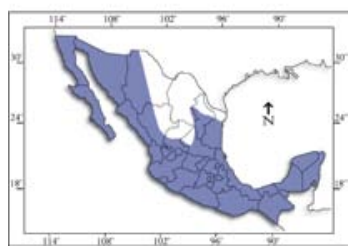

Lasiurus borealis

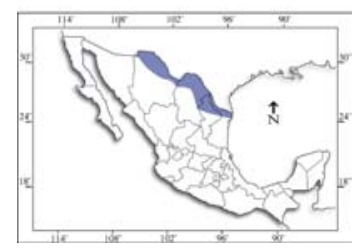

Lasiurus cinereus

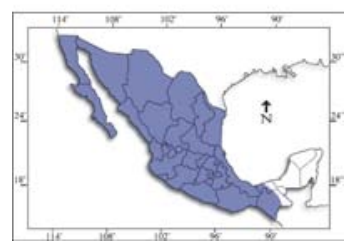

Lasiurus ega

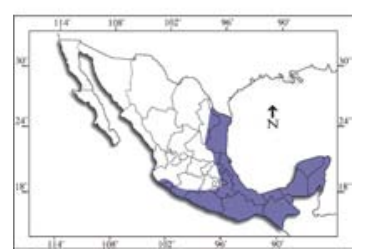

Lasiurus intermedius

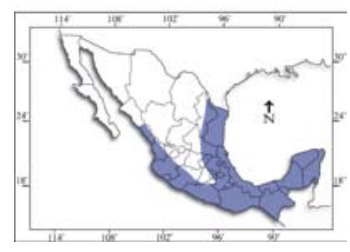

Lasiurus xanthinus

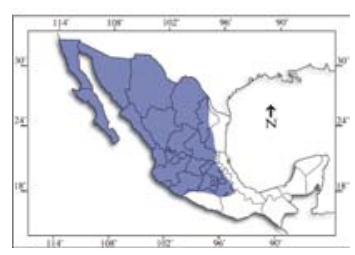

Nycticeius humeralis

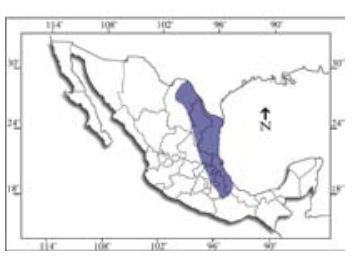

Parastrellus hesperus

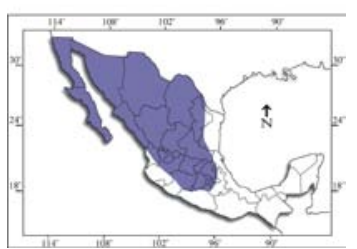

Perimyotis subflavus

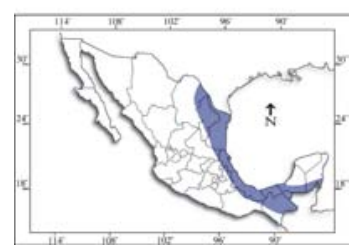

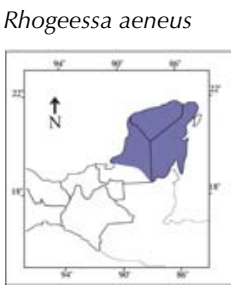

Rhogeessa bickhami

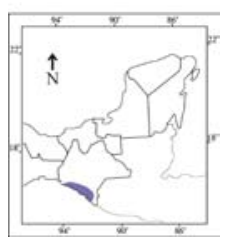

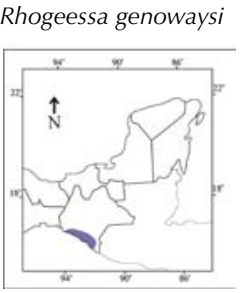

Rhogeessa mira

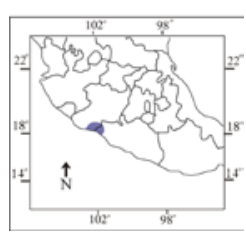


ORDEN CHIROPTERA

FAMILIA ANTROZOIDAE

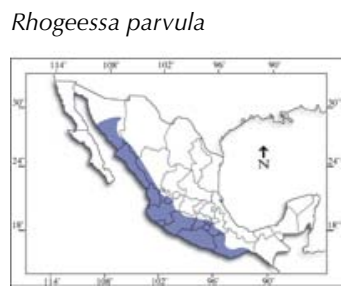

Rhogeessa tumida

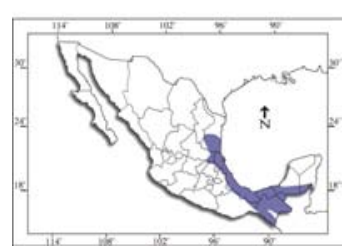

Antrozous pallidus

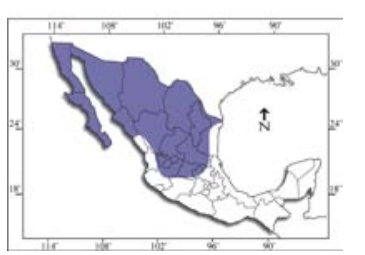

Bauerus dubiaquercus

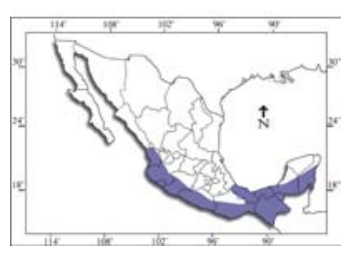

